\title{
3. VARIATIONS IN ARC VOLCANISM AND SEDIMENTATION RELATED TO RIFTING OF THE LAU BASIN (SOUTHWEST PACIFIC) ${ }^{1}$
}

\author{
Peter D. Clift $t^{2,3}$ and John E. Dixon ${ }^{2}$
}

\begin{abstract}
A series of six holes in the Lau Basin and two in the Tonga Platform were drilled during Leg 135 of the Ocean Drilling Program. The volcaniclastic sands found within the sedimentary cover are typically dominated by fragments of dacite glass, although basaltic andesite glass, calcic plagioclase, and ortho- and clinopyroxene grains are also identified. Total silica contents of individual glass grains indicate a bimodal spread of values at sites close to the Eastern Lau Spreading Center (ELSC), whereas those adjacent to the remnant arc (Lau Ridge) showed a complete spread of compositions. Analyses from the Tonga Platform (Site 840 ) indicate a prerift phase of arc volcanism (7.0-5.0 Ma) when a complete spectrum of silica values was erupted before backarc basin rifting occurred and the locus of volcanism switched to intrabasinal seamounts producing very similar island-arc tholeiites and their differentiates.

The principal mode of deposition of volcanic sediment in the Lau Basin is thought to be by proximal mass-flows and turbidity currents from submarine, intrabasinal seamounts. Continuous volcaniclastic sedimentation throughout the opening of the basin indicates that volcanism during the initial stages of basin rifting occurred in the form of intrabasinal submarine edifices, before the reestablishment of a fixed chain of arc volcanoes, adjacent to the Tonga Platform at approximately $3.0 \mathrm{Ma}$ in the southern Lau Basin. Renewal of arc volcanism approximately coincided with the propagation of backarc spreading centers into that area. Trace and rare earth element analyses of basaltic grains $\left(\mathrm{SiO}_{2}=45 \%-55 \%\right)$ from the Tonga Platform (Site 840$)$ before and after rifting show systematic trends in the abundances of incompatible elements and in incompatible element ratios that are consistent with either progressive depletion and then reenrichment of a mantle source, or an equivalent progressive increase and then decrease in the degree of melting. On top of this, there is some indication of a systematic shift in the character of enrichment towards a greater abundance of mobile incompatibles associated with the flux from the slab. The culmination of this cycle can be considered to be the modern Tofua Arc. These trends are most reasonably attributed to a thinning of the arc lithosphere before rifting and the associated increase in the height of the melting column within the subarc asthenosphere. Subsequent magmatic underplating after rifting causes a thickening of the arc lithosphere and a fall in the degree of partial melting, with a progressive slab-flux component being added to the source. The incompatible element ratio trends are correlated whether they are high-field-strength elements, rare-earth elements, or mobile large-ion-lithophile elements, implying that they relate to incompatibilities between source and melt, even though the absolute values in the basic rocks are typically arc-like rather than MORB-like, particularly in their enrichment in $\mathrm{Ba}$ and depletion in $\mathrm{Nb}$. The behavior of $\mathrm{Nb}$ in particular appears to have important implications for the process by which arc-tholeiites acquire their distinctive trace-element characteristics.
\end{abstract}

\section{INTRODUCTION}

The Lau Basin of the southwest Pacific Ocean has for some time now been regarded as a classic backarc basin, formed as a result of extension in a volcanically active setting along an active plate margin (e.g., Karig, 1970). The Lau Basin is a triangular shaped trough lying between two major, linear topographic highs, the Lau Ridge to the west and the Tofua Arc to the east (Fig. 1). The Lau Ridge is thought to represent a remnant Miocene Tonga Arc (e.g., Woodhall, 1985) that became inactive because of the Lau Basin rifting. The modern Tofua Arc represents the most recent phase of arc volcanism, and postdates rifting of the Lau Basin. Although rifting of the Lau Basin has dominated the Pliocene-Pleistocene history, the geology of the Tonga region records a considerably longer geologic history as subduction has been proceeding at least since the mid Eocene in the southwest Pacific (Burns and Andrews, 1973). After subduction initiation and foundation of an island-arc, the South Fiji Basin, a backarc basin, was formed by arc rifting during the Oligocene (35.5-26.0 Ma; Weissel and Watts, 1975). Subsequently, a period of steady-state subduction ensued along what was then a continuous Melanesian Arc (Gill et al., 1984), stretching all the way from New Zealand to Papua New

\footnotetext{
' Hawkins, J., Parson, L., Allan, J., et al., 1994. Proc. ODP, Sci. Results, 135: College Station, TX (Ocean Drilling Program).

${ }^{2}$ Department of Geology and Geophysics, The University of Edinburgh, West Mains Road, Edinburgh, EH9 37W, United Kingdom.

${ }^{3}$ Present address: Ocean Drilling Program, 1000 Discovery Drive, Texas A\&M University Research Park, College Station, TX 77845-9547, U.S.A.
}

Guinea. Collision of the Ontong-Java oceanic plateau with the New Hebrides section of the arc at 25.0 Ma (Moberly, 1971) caused a cessation of subduction and a reversal in its polarity in this area at 10.0 Ma (mid Miocene). This in turn resulted in a breakup of the continuous arc. Despite these events subduction continued in the same direction in Tonga and it was not until the uppermost Miocene (5.3 Ma) that this section of the arc rifted again to form the Lau Basin (Parson, Hawkins, Allan, et al., 1992). The Lau Basin has continued to widen up to the present day.

The geological evolution of the plate margin outlined above has resulted in the modern forearc to the Tofua Arc being marked by a broad (50-75 km across) platformal area, the Tonga Platform (Fig. 1). This is separated from the arc by a narrow trough, the Tofua Trough. On its trenchward side, the Tonga Platform falls rapidly away from its normal depths of $<1000 \mathrm{~m}$, to reach $>10,000 \mathrm{~m}$ in the trench itself (Tappin, 1992). The basement to the Tonga Platform is unknown, since the great sediment thicknesses deposited there preclude its sampling by drilling. However, some variety of arc crust, generated shortly after subduction initiation, seems the most likely candidate. This hypothesis was strengthened by the discovery of a pre-upper Eocene arc basement in the outer Tonga forearc (Parson, Hawkins, Allan, et al., 1992). The Tonga Platform is thus believed to have been in a forearc position adjacent to the Oligocene-Miocene Tonga Arc, now preserved as the Lau Ridge, before rifting of the Lau Basin.

The mechanism by which the Lau Basin rifted has been a subject of some controversy. Two models exist to explain its present geology. One model involves splitting and rifting along the axis of an original Miocene Tonga Arc (e.g., Karig, 1970). The new arc was then founded 


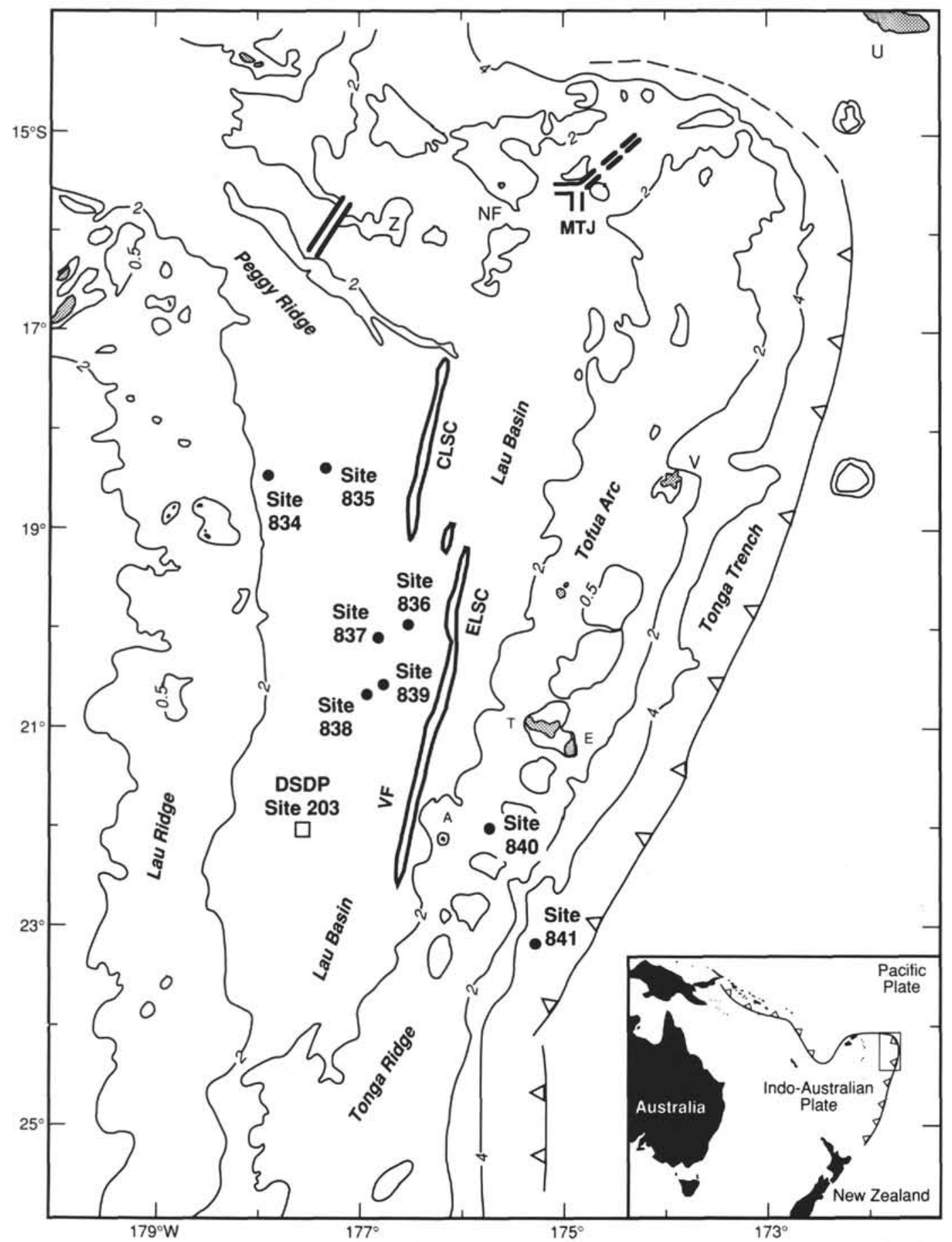

Figure 1. Outline map of the Lau Basin showing the positions of Site 834 to Site 840 . Bathymetry in kilometers. Line with triangles denotes plate boundary, with triangles on the overriding plate. $Z=Z$ ephyr Shoal, MTJ = Mangatolu Triple Junction, $\mathrm{NF}=$ Niuafo'ou, CLSC $=$ Central Lau Spreading Center, ELSC = Eastern Lau Spreading Center, VF = Valu Fa Ridge, $\mathrm{U}=$ Upolu, $\mathrm{V}=$ Vava'u, $\mathrm{T}=$ Tongatapu, $\mathrm{E}=$ 'Eua, $\mathrm{A}=$ 'Ata, $\mathrm{DSDP}=$ Deep Sea Drilling Project.

on one half of the rifted remains of the older edifice, leaving the other part as a remnant arc in the form of the Lau Ridge. A second model proposes that only the forearc region of the original island-arc is extended and a new arc is then created closer to the trench on rifted forearc material and/or new backarc basin crust (Hawkins et al., 1984; Hawkins and Melchior, 1985). Provisional interpretation of the Tonga forearc and Lau Basin drill sites from Ocean Drilling Program (ODP) Leg 135 suggests that the rifting involved extension of the arc crust trenchward of the volcanic front. Much of the western Lau Basin and basement of the Tofua Arc is probably composed of rifted OligoceneMiocene arc crust. The difference between the two models is thus seen to be minimal.

A significant amount of geological and geophysical data has already been collected around the Lau Basin, Tofua Arc and Tonga Platform. A combination of direct sampling, GLORIA sidescan sonar imaging (Parson et al., 1990) and other geophysical data indicate that the Lau Basin can be divided into two sections. The western Lau Basin, representing the uppermost Miocene-upper Pliocene history 
of the basin, comprises highs of rifted island arc crust separated by volcanically floored basins (Parson et al., 1992). The eastern Lau Basin, representing the upper Pliocene-Holocene history, comprises a series of grabens and horsts generated at a southward migrating oceanic spreading center. The sediments found within the Lau Basin thus provide an excellent opportunity to study the sedimentary response to arc rifting processes. In the case of the Tonga Platform, previous studies have included a number of dredging cruises (e.g., Scholl et al., 1985), as well as the collection of seismic profiles (e.g., Austin et al., 1989). These data indicate an area that has remained at a similar water depth since at least the middle Miocene (e.g., Clift, this volume), despite significant tectonic subsidence, caused by tectonic erosion of the underside of the forearc lithosphere. As a result the platform has collected large volumes of volcaniclastic debris, deposited against a background of nannofossil chalks. The location of the Tonga Platform in a forearc position relative to the modern and Miocene arcs means that the vast majority of volcaniclastic sedimentation on the platform must have been derived from the arc. Furthermore, its high standing topography makes the derivation of volcaniclastic material from the Lau Basin, or any other source, on to the platform difficult to achieve, thus minimizing the chance of contamination of the record. It is thus possible that the history of each arc may be preserved in some form within the sediments of the platform.

This paper attempts to describe the nature of sedimentation around the active volcanic arc and by inference its prerifting precursor, and to show how this is affected by the opening of a marginal basin. In addition, the sediments lying within the Lau Basin have been examined, to assess whether it is possible to separate the relative contributions of the Lau Ridge, the Tofua Arc and volcanic edifices within the Lau Basin, to sediment production within the backarc environment. The volcaniclastic sediments on the Tonga Platform (Site 840) can be used in conjunction with the volcanic rocks exposed on shore as a guide to the nature of island-arc volcanism, but a key question is: was this kind of volcanism distinctly different from that supplying sedi- ment within the basin itself, to the extent that geochemical criteria can back up sedimentologic evidence for provenance?

For this purpose sediment samples were taken from six sites drilled in the Lau Basin (Sites 834-839) and one in the adjacent Tonga Platform (Site 840; Fig. 1). The Lau Basin drill sites (Fig. 1) were all situated within small-scale (2-10 km wide), fault-bounded sub-basins and in all but one example (Site 838 ) penetrated igneous basement. The steep sides to the sub-basins, their small sizes and their partially filled nature suggest that sediment dispersal and deposition by waterborn means, either axially or laterally across the basin is, and would have been, very difficult. As far as the tectonic origin of each site is concerned, Site 836 is considered to be situated on crust generated at an oceanic spreading center (Parson et al., 1992; Parson and Hawkins, this volume). In contrast, Sites $834-835$ and $837-839$ are located within a zone of extended arc crust. Site 840 is situated east of the central Lau Basin, on the south central Tonga Platform, which is mildly deformed here by a number of normal faults of limited displacements.

\section{SEDIMENTARY FACIES}

The sequence of sedimentary facies deposited is remarkably consistent from one sub-basin to another throughout the backarc basin. The general pattern of sedimentation observed is characterized by a background of fine, clayey, nannofossil ooze, in which a series of volcaniclastic sand and silt beds was deposited, with both graded and massive varieties of sand and silt being identified. Each backarc site shows a progression to an decreasing proportion of volcaniclastic material upsection. The basal part of the section at each site is dominated by volcaniclastic turbidites and the upper part by pelagic nannofossil oozes (Fig. 2). This pattern is not uniform throughout the basin, as there are influxes of coarse volcaniclastic material within sections of nannofossil ooze, after the end of clastic-dominated sedimentation. At Site 837 there are three pulses of volcaniclastic influx observed, although again passing up into nannofossil ooze toward the

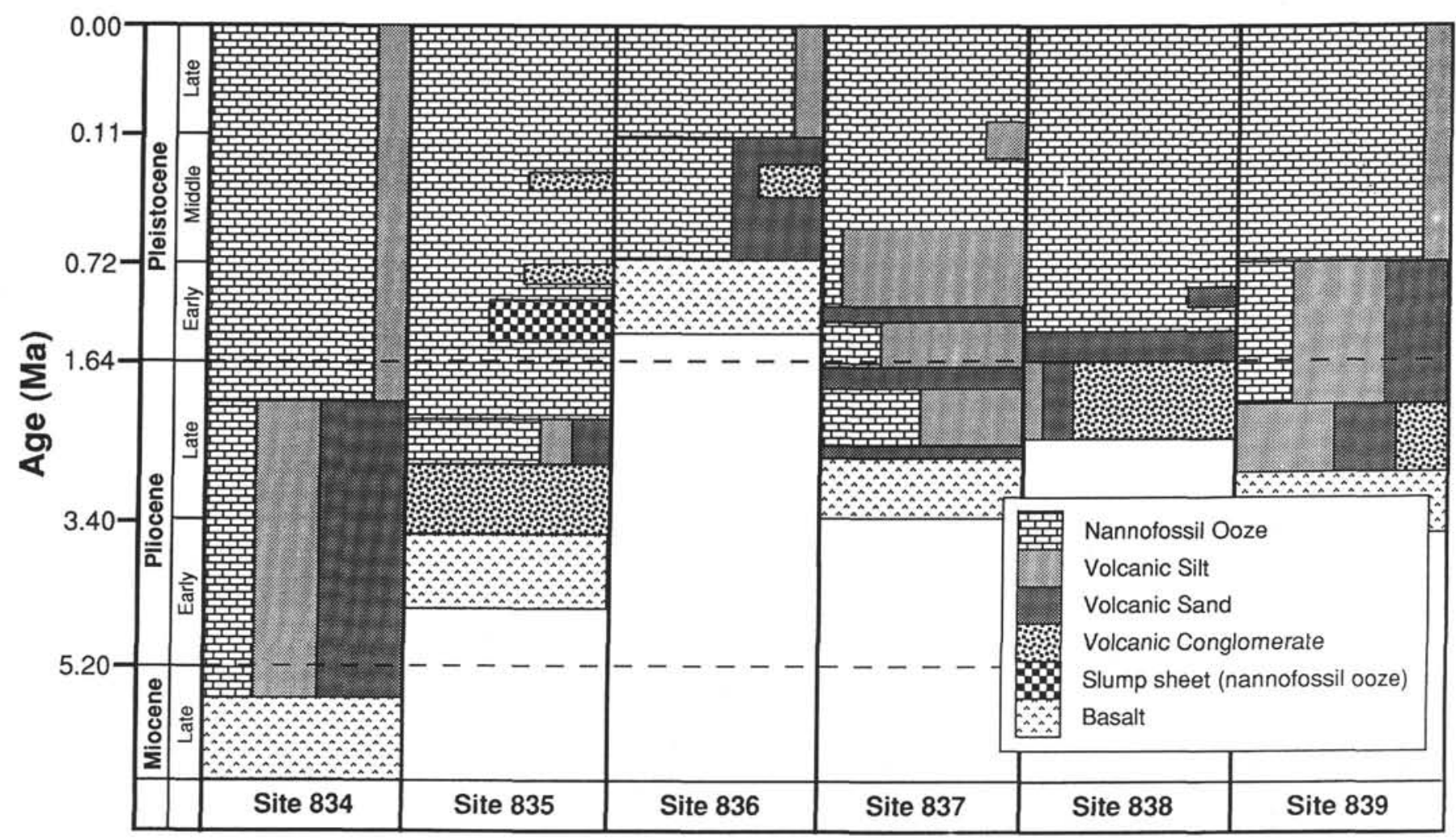

Figure 2. Summary diagram showing the dominant sedimentary facies deposited through time within the Lau Basin. 
top of the section. Critically, the upward transition from volcaniclasticdominated sediment to a pelagic-dominated section is not synchronous throughout the basin and is important evidence that sediment sources were locally controlled. Although a number of mass-flow conglomerates and coarse hyaloclastites were identified near the base of several sections, the majority of the sediment is made up of graded volcaniclastic turbidites and gravity flow sands. Sedimentary structures observed within the turbidites include sharp, erosive basal contacts, parallel lamination, and, more rarely, planar cross lamination, indicative of a current-deposited origin. The mass-flow sands are typically coarse-grained and massive, with occasional water escape structures. Grading is only poorly developed. Grain size, bed thicknesses, and facies suggest rapid sedimentation in a relatively proximal sedimentary setting, an interpretation supported by the individual grains, which are often texturally very immature. Delicate shards of volcanic glass and pumice are found and indicate that the mass-flow deposits were derived from a nearby, recently active volcanic source, without reworking over long distances. In marked contrast to this earlier sedimentary history, primary air-fall ash layers are identified with confidence towards the top of many of the backarc sections, corresponding to mid-late Pleistocene times. These differ from the epiclastic sediments in being very thin, generally less than $5 \mathrm{~cm}$ thick and marked by an absence of current-generated sedimentary structures. They are often composed of shards of only one glass type. Volumetrically, these ash layers and distributed pumice clasts form only a small proportion of the recovered sediment.

The sediments deposited at Site 840 on the Tonga Platform are also principally composed of mass-flow sands and conglomerates, as well as volcaniclastic turbidites. These were deposited against a background of pelagic nannofossil chalks, although without the hydrothermal manganese contamination seen in the Lau Basin itself. As they lie relatively close to the Tofua Arc today and were presumably close to the Miocene Tonga Arc (Lau Ridge), the largely proximal character of the sediments can be readily explained to be the result of the short transport distance from the source region (i.e., the volcanic arc). The overall sedimentary character of Site 840 shows a progressive fining upwards in the upper Miocene, before Lau Basin rifting at 5.25 Ma. Rifting itself is marked by the influx of coarse volcaniclastic conglomerates, after which the sediments rapidly fine upward. The Pliocene-Pleistocene is typified by a carbonate rich sequence, with interbedded minor ash layers and volcanic sands.

\section{Shipboard Analysis}

As well as being routinely described as part of the standard onboard core analysis, volcaniclastic sediments were also examined as grain mounts. A selected number of layers from throughout the recovered sections at each site were sampled for shipboard analysis. These were disaggregated and sieved. The $<38 \mu \mathrm{m}$ fraction was taken and the refractive indices of the constituent glass grains were determined using a reference set of optical refractive index oils (e.g., Parson, Hawkins, Allan, et al., 1992, pp. 105-109). These values were then used to determine the total silica contents of the glasses, using the formula of Church and Johnson (1980).

Within the backarc basin, this refractive index data typically indicate a mixed sediment source, resolvable into a bimodal suite of basaltic andesite $\left(\mathrm{SiO}_{2}=52 \%-56 \%\right)$ and dacitic $\left(\mathrm{SiO}_{2}=68 \%-73 \%\right)$ glasses (e.g., Parson, Hawkins, Allan, et al., 1992, pp. 301-304). Only a limited amount of intermediate material was identified, except in the case of the Tonga Platform (Site 840), where a significant population was identified between $58 \%$ and $62 \% \mathrm{SiO}_{2}$ (Parson, Hawkins, Allan, et al., 1992, pp. 512-518). Although silica-poor sediment grains were identified, a large majority of the sediment grains were thought to be of dacitic composition. Microprobe analyses, therefore, were undertaken in the laboratory to test and extend these findings.

\section{Sample Collection and Preparation}

Samples were chosen from discrete volcaniclastic layers and were preferentially taken from the coarser grained bases of the layer where this was possible. Because Site 838 is thought to lie in the same sub-basin as Site 839 on the basis of the seismic profiling evidence (Parson, Hawkins, Allan, et al., 1992, pp. 414-417), relatively few analyses were made on grains from this site, in order to maximize coverage across the basin.

Samples that were partially or fully indurated were impregnated with epoxy resin, after which double-polished, 1-in.-wide probe thin sections were prepared. Less well indurated sediments were disaggregated by being mixed with water and placed in an ultrasonic bath for $24 \mathrm{hr}$. After this the sediments were sieved through 125-, 63-, and $45-\mu \mathrm{m}$ sized grilles and washed by a high pressure water jet. A selection of grains from the largest size fraction $(>125 \mu \mathrm{m})$ was then mounted in epoxy and polished, using first $6 \mu \mathrm{m}$ and then $1 \mu \mathrm{m}$-sized diamond paste. The slides were carbon-coated before electron probe analysis.

\section{Analytical Procedure}

The samples were analyzed using a Cameca Camebax Microbeam electron microprobe for a series of ten major elements: $\mathrm{Si}, \mathrm{Al}, \mathrm{Na}, \mathrm{K}$, $\mathrm{Ca}, \mathrm{Mg}, \mathrm{Fe}, \mathrm{P}, \mathrm{Ti}, \mathrm{Mn}$, and, in some cases, $\mathrm{Cr}$. A beam current of 10 $\mathrm{nA}$ was employed, operating at a voltage of $20 \mathrm{kV}$. A count time of 30 sec. was used for each element. Between 4 and 6 grains were analyzed from each sediment sample, with an attempt being made, through the use of a Link AN10/85S energy dispersive spectrometer (EDS), to probe at least one of each grain type encountered, although with special emphasis on volcanic glass shards. A limited amount of data was also obtained from the plagioclase and pyroxene grains.

Samples of basaltic and basaltic andesite glass identified by the electron probe study were subsequently cleaned, coated in gold and analyzed using the Cameca ims $4 \mathrm{f}$ ion microprobe at Edinburgh University, running Charles Evans and Associates software, for a suite of 20 trace and rare earth elements (REEs). The mafic glasses were chosen for trace element analysis to minimize the geochemical effect of fractional crystallization and thus yield the greatest amount of information concerning the petrogenetic history and source characteristics of the magmas. Electron backscattered images of glass obtained on the electron microprobe often showed the presence of microphenocrysts of plagioclase within the glass. Where possible, the glass grains analyzed by the ion microprobe were aphyric or contained the minimum number of phenocrysts within the available sample set. There is no way of establishing whether phenocryst phases were actually absent from the host magma of an aphyric glass fragment on eruption.

\section{MAJOR ELEMENT CHEMISTRY}

\section{Volcanic Glass}

Representative chemical analyses for the volcanic glass grains probed are displayed in Tables 1-3. The reliability of the data is a key question. Visually, very few of the grains show any cloudiness attributable to hydration. Where this is seen it is from samples from the base of a section, supporting the contention that it reflects alteration. Low analytical totals can arise from an indigenous volatile content in the melt or from subsequent hydration, possibly without accompanying visual evidence. Primary water contents in the melt may be expected to rise during crystal fractionation (e.g., Burnham and Jahns, 1962) but in a varied suite of lavas evolving under different conditions of volatile degassing, and perhaps differing primary water contents, a range of volatile contents might be expected over much of the compositional spread, with a broad correlation between volatile-loss measured by [100\% - Total], and silica content. Figure 3 shows this 
Table 1. Representative microprobe analyses of intermediate and acidic volcanic glass grains from each of the Lau Basin and Tonga Platform sites (834-84).

\begin{tabular}{|c|c|c|c|c|c|c|c|c|c|c|c|c|c|c|c|c|c|c|}
\hline Hole: & $834 \mathrm{~A}$ & $834 \mathrm{~A}$ & $834 \mathrm{~A}$ & $834 \mathrm{~A}$ & $834 \mathrm{~A}$ & $834 \mathrm{~A}$ & $835 \mathrm{~A}$ & $835 \mathrm{~A}$ & $835 \mathrm{~A}$ & $835 \mathrm{~A}$ & $835 \mathrm{~A}$ & $836 \mathrm{~A}$ & $836 \mathrm{~A}$ & $837 \mathrm{~A}$ & $837 \mathrm{~A}$ & $837 \mathrm{~A}$ & $837 \mathrm{~A}$ & $837 \mathrm{~A}$ \\
\hline Core, section: & $1 \mathrm{H}-2$ & $1 \mathrm{H}-3$ & $2 \mathrm{H}-1$ & $5 \mathrm{H}-2$ & $7 \mathrm{H}-4$ & $11 X-1$ & $2 \mathrm{H}-1$ & $7 \mathrm{H}-3$ & $7 \mathrm{H}-3$ & $8 \mathrm{H}-2$ & $8 \mathrm{H}-2$ & $2 \mathrm{H}-6$ & $3 \mathrm{H}-6$ & $2 \mathrm{H}-4$ & $2 \mathrm{H}-7$ & $6 \mathrm{H}-4$ & $9 \mathrm{H}-1$ & $9 \mathrm{H}-3$ \\
\hline Interval $(\mathrm{cm})$ : & 37 & 82 & 37 & 20 & 78 & 40 & 79 & 140 & 140 & 140 & 140 & 34 & 134 & 103 & 50 & 132 & 53 & 93 \\
\hline Age (k.y.): & 450 & 470 & 600 & 2600 & 3300 & 3650 & 400 & 1350 & 1350 & 1550 & 1550 & 400 & 600 & 600 & 650 & 1600 & 1825 & 2050 \\
\hline Depth (mbsf): & 1.87 & 3.82 & 8.79 & 39.30 & 60.38 & 93.60 & 10.29 & 61.40 & 61.40 & 69.40 & 69.40 & 9.08 & 19.43 & 13.53 & 17.50 & 51.8 & 67 & 78.43 \\
\hline \multicolumn{19}{|c|}{ Major elements (wt $\%$ ): } \\
\hline $\mathrm{SiO}_{2}$ & 66.34 & 61.85 & 63.09 & 72.68 & 54.93 & 75.52 & 56.76 & 58.46 & 64.59 & 60.32 & 59.39 & 56.95 & 56.66 & 63.65 & 61.10 & 76.52 & 77.45 & 56.14 \\
\hline $\mathrm{Al}_{2} \mathrm{O}_{3}$ & 12.99 & 12.58 & 13.60 & 10.88 & 14.63 & 11.22 & 17.59 & 13.91 & 13.15 & 13.38 & 13.68 & 13.66 & 14.10 & 13.46 & 12.23 & 11.28 & 10.99 & 14.14 \\
\hline $\mathrm{MgO}$ & 1.58 & 2.46 & 2.05 & 0.69 & 5.26 & 0.17 & 1.18 & 2.45 & 1.32 & 1.93 & 2.15 & 3.40 & 2.74 & 1.68 & 3.51 & 0.42 & 0.36 & 3.04 \\
\hline $\mathrm{Fe}_{2} \mathrm{O}_{3}$ & 7.52 & 9.87 & 7.29 & 5.19 & 6.90 & 3.39 & 6.55 & 8.91 & 6.47 & 8.55 & 8.45 & 10.48 & 9.82 & 8.23 & 11.95 & 2.33 & 1.65 & 10.38 \\
\hline $\mathrm{CaO}^{3}$ & 5.57 & 7.12 & 5.78 & 3.84 & 8.48 & 1.86 & 7.75 & 6.54 & 4.56 & 5.86 & 6.23 & 8.00 & 6.93 & 6.10 & 8.10 & 2.38 & 1.47 & 1.28 \\
\hline $\mathrm{Na}_{2} \mathrm{O}$ & 1.56 & 1.96 & 2.29 & 1.10 & 3.00 & 1.12 & 2.85 & 1.64 & 1.72 & 1.68 & 1.62 & 1.97 & 1.81 & 1.35 & 1.42 & 0.99 & 0.86 & 0.46 \\
\hline $\mathrm{K}_{2} \mathrm{O}$ & 1.25 & 0.73 & 0.58 & 0.78 & 2.36 & 0.38 & 0.80 & 0.92 & 1.11 & 1.04 & 0.94 & 0.72 & 0.38 & 0.47 & 0.36 & 0.73 & 0.98 & 7.90 \\
\hline $\mathrm{TiO}_{2}$ & 0.66 & 0.48 & 0.95 & 0.44 & 0.94 & 0.28 & 0.80 & 1.23 & 1.08 & 1.24 & 1.23 & 0.94 & 1.08 & 0.76 & 0.83 & 0.42 & 0.32 & 1.14 \\
\hline $\mathrm{P}_{2} \mathrm{O}_{5}$ & 0.31 & 0.19 & 0.27 & 0.19 & 0.41 & 0.07 & 0.31 & 0.53 & 0.35 & 0.41 & 0.38 & 0.28 & 0.32 & 0.19 & 0.23 & 0.09 & 0.05 & 0.27 \\
\hline MnO & 0.17 & 0.14 & 0.18 & 0.16 & 0.22 & 0.14 & 0.13 & 0.18 & 0.21 & 0.21 & 0.21 & 0.17 & 0.22 & 0.21 & 0.32 & 0.06 & 0.09 & 0.19 \\
\hline Total & 97.97 & 97.39 & 96.08 & 95.78 & 96.91 & 94.14 & 94.72 & 94.78 & 94.55 & 94.61 & 94.29 & 96.56 & 94.06 & 96.09 & 100.04 & 95.22 & 94.22 & 94.94 \\
\hline
\end{tabular}

Table 1 (continued).

\begin{tabular}{|c|c|c|c|c|c|c|c|c|c|c|c|c|c|c|c|c|c|c|}
\hline Hole: & $839 \mathrm{~A}$ & $839 \mathrm{~A}$ & $839 \mathrm{~A}$ & $839 \mathrm{~A}$ & $839 \mathrm{~A}$ & $839 \mathrm{~A}$ & $839 \mathrm{~A}$ & $840 \mathrm{~A}$ & $840 \mathrm{~A}$ & $840 \mathrm{C}$ & $840 B$ & $840 \mathrm{~B}$ & $840 \mathrm{~B}$ & $840 \mathrm{~B}$ & $840 \mathrm{~A}$ & $840 \mathrm{C}$ & $840 \mathrm{~B}$ & $840 \mathrm{~B}$ \\
\hline Core, section: & $4 \mathrm{H}-3$ & SH-2 & $6 \mathrm{H}-1$ & $4 \mathrm{H}-4$ & $6 \mathrm{H}-1$ & $7 \mathrm{H}-2$ & $11 \mathrm{H}-6$ & $1 \mathrm{H}-\mathrm{I}$ & $1 \mathrm{H}-1$ & $2 \mathrm{H}-1$ & $17 \mathrm{X}-\mathrm{CC}$ & $43 X-3$ & $51 X-5$ & $55 \mathrm{X}-4$ & $1 \mathrm{H}-3$ & $1 \mathrm{H}-2$ & $12 X-5$ & $61 X-3$ \\
\hline Interval $(\mathrm{cm})$ : & 35 & 15 & 117 & 38 & 117 & 101 & 49 & 102 & 102 & 37 & 2 & 133 & 10 & 94 & 45 & 114 & 50 & 131 \\
\hline Age (k.y.): & 920 & 1100 & 1400 & 920 & 1400 & 1700 & 1700 & 350 & 350 & 1800 & 5125 & 6130 & 6500 & 6700 & 450 & 1725 & 5000 & 6950 \\
\hline Depth (mbsf): & 28.35 & 34.65 & 43.67 & 28.35 & 43.67 & 54.51 & 99.49 & 1.02 & 1.02 & 47.87 & 153.82 & 398.33 & 477.80 & 515.42 & 3.50 & 40.64 & 110.48 & 572.61 \\
\hline \multicolumn{19}{|c|}{ Major elements (wt\%): } \\
\hline $\mathrm{Si}_{2} \mathrm{O}$ & 65.50 & 63.70 & 57.07 & 65.50 & 59.34 & 74.37 & 76.35 & 63.47 & 63.47 & 71.50 & 67.01 & 66.37 & 71.16 & 63.93 & 56.50 & 55.28 & 54.48 & 50.82 \\
\hline $\mathrm{Al}_{2} \mathrm{O}_{3}$ & 14.57 & 11.67 & 13.15 & 14.57 & 14.67 & 11.53 & 11.11 & 14.21 & 14.21 & 11.58 & 15.98 & 14.83 & 10.45 & 20.06 & 11.89 & 12.75 & 12.72 & 16.52 \\
\hline $\mathrm{MgO}^{2}$ & 0.65 & 1.65 & 2.60 & 0.65 & 2.15 & 0.47 & 0.31 & 1.34 & 1.34 & 0.76 & 0.95 & 0.33 & 0.49 & 0.41 & 4.38 & 4.05 & 4.48 & 2.78 \\
\hline $\mathrm{Fe}_{2} \mathrm{O}_{3}$ & 5.79 & 9.52 & 10.40 & 5.79 & 8.53 & 2.61 & 3.36 & 8.16 & 8.16 & 4.88 & 5.95 & 5.04 & 6.96 & 1.57 & 14.06 & 14.23 & 13.23 & 11.33 \\
\hline $\mathrm{Na}_{2} \mathrm{O}^{3}$ & 6.35 & 5.84 & 6.92 & 2.39 & 0.97 & 2.67 & 2.85 & 2.11 & 5.52 & 1.01 & 3.32 & 4.78 & 2.96 & 2.68 & 1.13 & 1.92 & 2.09 & 4.69 \\
\hline $\mathrm{K}_{2} \mathrm{O}$ & 2.39 & 1.90 & 1.45 & 0.61 & 0.72 & 0.99 & 1.12 & 1.60 & 2.11 & 0.27 & 1.09 & 3.06 & 1.07 & 3.20 & 0.91 & 0.92 & 1.55 & 1.71 \\
\hline $\mathrm{CaO}$ & 0.61 & 0.68 & 0.97 & 6.35 & 6.70 & 0.80 & 0.59 & 5.52 & 1.60 & 3.73 & 4.43 & 0.98 & 1.72 & 8.23 & 9.25 & 8.89 & 5.57 & 10.44 \\
\hline $\mathrm{TiO}_{2}$ & 0.82 & 1.04 & 1.28 & 0.82 & 1.08 & 0.43 & 0.37 & 0.71 & 0.71 & 0.69 & 0.86 & 0.87 & 1.47 & 0.11 & 1.27 & 1.31 & 1.32 & 1.29 \\
\hline $\mathrm{P}_{2} \mathrm{O}_{5}^{2}$ & 0.25 & 0.24 & 0.38 & 0.25 & 0.39 & 0.13 & 0.15 & 0.23 & 0.23 & 0.23 & 0.28 & 0.40 & 0.54 & 0.07 & 0.34 & 0.36 & 0.37 & 0.21 \\
\hline $\mathrm{MnO}$ & 0.14 & 0.15 & 0.18 & 0.14 & 0.14 & 0.08 & 0.10 & 0.17 & 0.17 & 0.14 & 0.14 & 0.15 & 0.16 & 0.07 & 0.27 & 0.28 & 0.26 & 0.21 \\
\hline Total & 97.05 & 96.40 & 94.40 & 97.05 & 94.69 & 94.10 & 96.33 & 97.51 & 97.51 & 94.78 & 99.85 & 96.80 & 96.97 & 100.32 & 99.78 & 97.33 & 96.08 & 95.27 \\
\hline
\end{tabular}

Note: Analyses in the box show representatives of the high alkali/low silica lavas found at Site 840 .

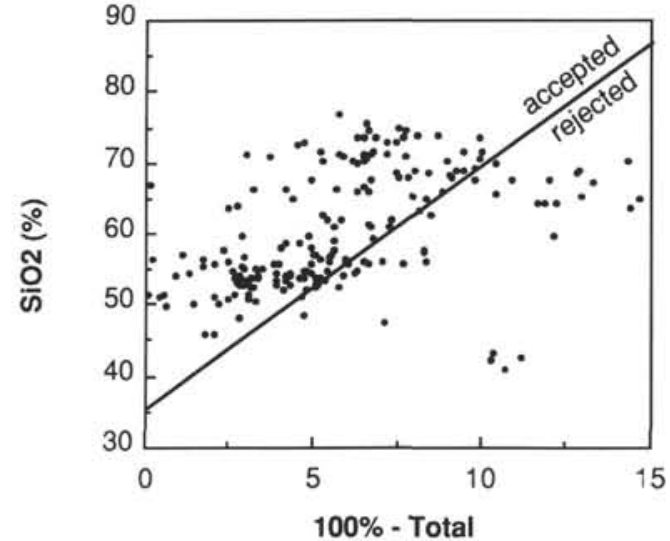

Figure 3. Diagram showing the relationship between total silica contents of volcanic glass shards from Site 840 with their apparent volatile contents expressed as $100 \%$ analytical total.

to be the case. The data has a slope that corresponds to the effective upper limit of $\mathrm{SiO}_{2}$ in a granitic liquid when subject to a variable addition of volatiles. This plot allows very anomalous low totals at a particular silica content to be identified and rejected as probably subject to secondary hydration.

An important additional source of error and uncertainty is the loss of sodium under the electron beam. This is well known (e.g., Neilson and Sigurdsson, 1981) and can be clearly seen on Figure 4. The expected levels of total alkalies at around $8 \%$ in the most siliceous

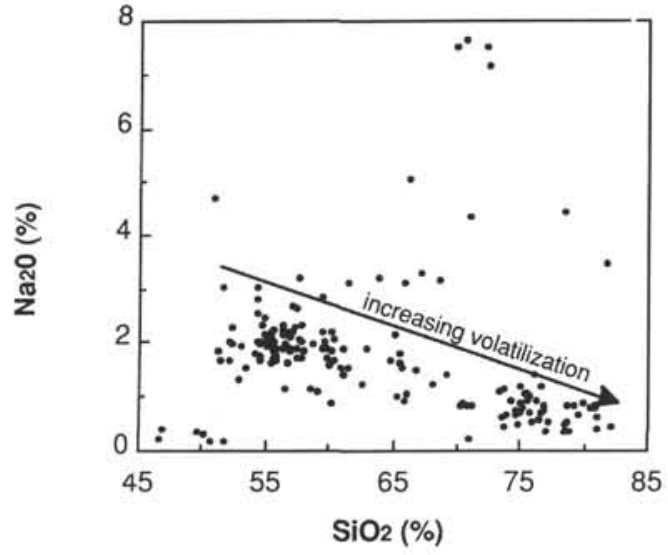

Figure 4. Diagram showing the relationship between analyzed total silica and $\mathrm{Na}_{2} \mathrm{O}$ contents. The decrease in $\mathrm{Na}_{2} \mathrm{O}$ with increasing silica reflects the volatilization of high silica glasses under the microprobe beam.

glasses are not achieved, because sodium declines sharply with silica content from basaltic andesite compositions onwards. Potassium values are scattered but not so conspicuously subject to loss.

Sodium loss under the beam causes the apparent concentration of other elements to rise. As sodium loss is related to silica content and is not subject to a constant loss factor, and volatile content is also broadly related to silica, the two effects counteract each other and measured abundances of most elements, except sodium, are probably not far from their volatile-free levels. The upper bound of the data on Figure 3, 
Table 2. Representative microprobe analyses of basaltic and basaltic andesite volcanic glass grains from each of the Lau Basin sites (834-839).

\begin{tabular}{|c|c|c|c|c|c|c|c|c|c|c|c|c|c|c|c|c|c|c|c|c|c|}
\hline Hole: & $834 \mathrm{~A}$ & $834 \mathrm{~A}$ & $834 \mathrm{~A}$ & $834 \mathrm{~A}$ & $834 \mathrm{~A}$ & $834 \mathrm{~A}$ & $835 \mathrm{~A}$ & $835 \mathrm{~A}$ & $835 \mathrm{~A}$ & $835 \mathrm{~A}$ & $835 \mathrm{~A}$ & $835 \mathrm{~A}$ & $836 \mathrm{~A}$ & $836 \mathrm{~A}$ & $836 \mathrm{~A}$ & $837 \mathrm{~A}$ & $837 \mathrm{~A}$ & $837 \mathrm{~A}$ & $837 \mathrm{~A}$ & $837 \mathrm{~A}$ & $837 \mathrm{~A}$ \\
\hline Core, section: & $2 \mathrm{H}-1$ & $3 \mathrm{H}-1$ & $7 \mathrm{H}-4$ & $9 \mathrm{H}-3$ & $10 \times-3$ & $10 \mathrm{X}-\mathrm{CC}$ & $4 \mathrm{H}-3$ & $4 \mathrm{H}-5$ & $8 \mathrm{H}-6$ & $10 \mathrm{H}-1$ & $10 \mathrm{H}-1$ & $16 \mathrm{H}-3$ & $1 \mathrm{H}-1$ & $2 \mathrm{H}-6$ & $3 \mathrm{H}-6$ & $1 \mathrm{H}-3$ & $3 \mathrm{H}-1$ & $3 \mathrm{H}-2$ & $4 \mathrm{H}-6$ & $4 \mathrm{H}-6$ & $4 \mathrm{H}-6$ \\
\hline Interval $(\mathrm{cm})$ : & 37 & 72 & 78 & 98 & 102 & 13 & 45 & 57 & 25 & 26 & 26 & 100 & 84 & 38 & 123 & 86 & 86 & 124 & 52 & 52 & 67 \\
\hline Age (k.y.) & 450 & 1000 & 3300 & 3500 & 3570 & 3600 & 850 & 860 & 2000 & 2400 & 2400 & 2800 & 100 & 400 & 600 & 450 & 700 & 710 & 900 & 900 & 900 \\
\hline Depth (mbsf): & 1.87 & 17.82 & 60.38 & 78.08 & 87.62 & 88.14 & 31.95 & 35.07 & 74.25 & 85.67 & 85.67 & 146.5 & 0.84 & 9.08 & 19.43 & 3.86 & 18.36 & 20.24 & 35.02 & 35.02 & 35.17 \\
\hline \multicolumn{22}{|c|}{ Major elements (wt\%): } \\
\hline $\mathrm{SiO}_{2}$ & 54.61 & 53.28 & 54.93 & 57.25 & 54.51 & 52.25 & 54.56 & 49.19 & 55.07 & 48.83 & 46.30 & 59.31 & 53.93 & 54.50 & 53.48 & 52.79 & 53.88 & 51.98 & 50.36 & 51.50 & 51.98 \\
\hline $\mathrm{Al}_{2} \mathrm{O}_{3}$ & 14.38 & 13.54 & 14.63 & 14.10 & 14.74 & 13.47 & $\begin{array}{l}14.68 \\
0.74\end{array}$ & 15.02 & $\begin{array}{l}14.15 \\
0.92\end{array}$ & 15.09 & 18.06 & 13.48 & 14.61 & 14.41 & 13.83 & 14.78 & 14.03 & 14.74 & 14.44 & 14.57 & 14.09 \\
\hline $\mathrm{Fe}_{2} \mathrm{O}_{3}$ & 10.11 & $\begin{array}{c}12.11 \\
5.05\end{array}$ & $\begin{array}{r}6.90 \\
5\end{array}$ & 9.29 & $\begin{array}{r}10.33 \\
3.30\end{array}$ & 11.60 & 9.74 & $\begin{array}{r}8.83 \\
7.43\end{array}$ & 9.83 & 10.54 & 6.55 & 9.10 & 10.26 & 9.89 & 11.79 & 10.12 & 11.00 & 10.34 & 9.36 & 9.79 & 8.59 \\
\hline $\mathrm{MgO}$ & $\begin{array}{l}5.17 \\
081\end{array}$ & $\begin{array}{r}5.05 \\
1047\end{array}$ & 5.26 & 2.89 & $\begin{array}{l}3.39 \\
700\end{array}$ & 6.00 & 3.50 & 7.43 & 3.42 & 6.49 & 6.81 & 1.41 & 5.09 & 4.62 & 3.22 & 5.58 & 462 & 5.94 & 7 & 5.36 & 6.71 \\
\hline $\mathrm{CaO}$ & $\begin{array}{r}9.81 \\
180\end{array}$ & 10.47 & 8.48 & 6.89 & 7,92 & 10.72 & 7.89 & 13.14 & 8.50 & 11.86 & 14.17 & 5.23 & 9.92 & 9.56 & 7.59 & 10.21 & 9.58 & 10.74 & 11.22 & 10.40 & 11.72 \\
\hline $\mathrm{Na}_{2} \mathrm{O}$ & $\begin{array}{l}1.89 \\
8.5\end{array}$ & & & 1.65 & 1.99 & 1.71 & 1.36 & 1.48 & 1.25 & 3.14 & 2.20 & 1.42 & 1.89 & 1.53 & & 136 & & 2.11 & 167 & 2.19 & 1.56 \\
\hline $\mathrm{K}_{2} \mathrm{O}$ & 0.52 & 0.41 & 2.36 & 0.43 & 0.52 & 0.14 & 0.40 & 0.33 & 0.22 & 0.14 & 0.09 & 0.66 & 0.50 & 0.44 & 0.27 & 0.58 & 0. & 0.25 & 0.32 & 0.41 & 0.32 \\
\hline $\mathrm{TiO}_{2}$ & 0.69 & 0.72 & 0.94 & 1.01 & 1.09 & 0.87 & 1.36 & 0.67 & 0.82 & 0.95 & 0.93 & 1.03 & 0.70 & 0.57 & 18 & 0.65 & & 1.15 & 074 & 101 & 0.77 \\
\hline & 0.26 & 0.25 & 0.41 & 0.32 & 0.32 & 0.24 & 0.30 & 0.33 & 0.21 & 0.14 & 0.29 & 0.44 & 0.27 & 0.27 & & 0.31 & & 0.30 & & 0.37 & 0.33 \\
\hline $\mathrm{MnO}$ & 0.21 & 0.21 & 0.22 & 0.19 & 0.23 & 0.20 & 0.21 & 0.20 & 0.22 & 0.20 & 0.14 & & & 0.19 & & 0.20 & & 0.19 & & 0.02 & 0.17 \\
\hline $\mathrm{Cr}_{2} \mathrm{O}_{3}$ & & & & & & & & & & & & & & & & & & & 0.05 & 0.00 & \\
\hline Total & 97.65 & 97.32 & 97.13 & 94.01 & 95.04 & 97.20 & 94.00 & 96.62 & 93.71 & 97.39 & 95.51 & 92.28 & 97.34 & 95.99 & 94.25 & 96.57 & 96.03 & 97.74 & 94.94 & 95.61 & 96.24 \\
\hline \multicolumn{22}{|c|}{ Trace elements (ppm): } \\
\hline $\mathrm{Rb}$ & & 2.9 & 51.4 & 2.7 & 4.6 & 1.1 & 4.4 & 4.4 & 1.1 & 1.7 & 0.2 & 9.5 & 4.4 & 3.8 & 2.5 & 5.1 & 0.5 & 6.4 & 5.4 & 5.4 & 4.4 \\
\hline $\mathrm{Sr}$ & 148 & 143 & 305 & 164 & 200 & 117 & 138 & 153 & 104 & 190 & 197 & 111 & 181 & 170 & 107 & 196 & 86 & 158 & 209 & 209 & 192 \\
\hline$Y$ & 10.5 & 11.0 & 22.4 & 20.2 & 19.6 & 16.4 & 23.1 & 11.7 & 13.5 & 23.0 & 14.3 & 35.7 & 13.2 & 11.0 & 26.7 & 14.2 & 12.1 & 20.3 & 16.9 & 16.9 & 16.5 \\
\hline $\mathrm{Zr}$ & 21 & 17 & 62 & 58 & 53 & 36 & 68 & 18 & 33 & 74 & 39 & 112 & 32 & 23 & 84 & 30 & 29 & 53 & 55 & 55 & 41 \\
\hline $\mathrm{N}$ & 0.14 & 0.02 & 1.08 & 0.44 & 0.57 & 0.00 & 0.91 & 0.16 & 0.05 & 0.35 & 0.20 & 1.59 & 0.13 & 0.03 & 1.46 & 0.07 & 0.00 & 0.54 & 0.54 & 0.54 & 0.39 \\
\hline $\mathrm{B}$ & 99 & 77 & 359 & 179 & 135 & 28 & 55 & 38 & 66 & 66 & 38 & 69 & 133 & 110 & 50 & 135 & 92 & 85 & 52 & 52 & 45 \\
\hline L & 1.9 & 1.4 & 7.0 & 2.9 & 30 & 1 & 4.3 & 2.4 & 1.1 & 5.1 & 3.1 & 6.7 & 2.2 & 1.7 & 3.5 & 1.9 & 1.0 & 4.1 & 4.6 & 4.6 & 3.0 \\
\hline c & 4. & 3.2 & 16 & 8 & 8 & & 10.1 & 5.0 & 3.2 & 9.5 & 5.1 & 15.5 & 4.9 & 3.8 & 9. & 5. & & 10. & 10.4 & 10.4 & 8.1 \\
\hline $\mathrm{N}$ & 4 & 2.8 & 12 & 6 & 8.2 & & 9.3 & 4.3 & 3. & 9.4 & 5.4 & 14.1 & 4.8 & 3.5 & 8.8 & 4. & & 8 & 8 & $\gamma$ & 6.6 \\
\hline & & 1. & & & & & 2. & 1. & 1. & 2. & 1.7 & 4.2 & 1. & 1.2 & 2. & 1. & & & 2. & 2.5 & 1.8 \\
\hline E & & 0.20 & & & & & 1.18 & 0.54 & 0.50 & 0.90 & 0.60 & 1.43 & 0.61 & 0.38 & 1.04 & 0.46 & 0.48 & 0.82 & 0.82 & 0.82 & 0.58 \\
\hline $\mathrm{T}$ & & 0.2 & 0 . & & & & 0. & 0. & 0 & 0. & 0. & 1.0 & 0.4 & 0.3 & 0.7 & 0. & 0.3 & 0.5 & 0.6 & 0.6 & 0.5 \\
\hline D & & 1. & & & & & 4. & 2 & 2 & 3 & 2. & 6. & 2 . & 2. & 5. & 2. & & & & 3. & 2.7 \\
\hline H & & 0. & 0. & & & & & 0 & 0 & 0.73 & 0. & 1. & 0.56 & 0.46 & 1.06 & 0.44 & 0 . & 0.73 & 0.62 & 0.62 & 0.63 \\
\hline E & 2. & 1.0 & 2.2 & 1. & 1.4 & 1.7 & 2. & 1. & 1. & 2. & 1. & 4. & 1. & & 3. & 1. & 1. & 1.9 & 2. & 2.3 & 1.7 \\
\hline Lu & 0.40 & 0.14 & 0.35 & 0.21 & 0.17 & 0.33 & 0. & 0 . & 0 & 0 & 0. & 0 & 0. & 0.2 & 0 . & 0.24 & 0.3 & 0.31 & 0.25 & 0.25 & 0.28 \\
\hline $\mathrm{Hf}$ & 2.36 & 0.38 & 1.48 & 1.01 & 0.96 & 1.01 & 2. & 0 & 1. & 1 & 0 & 2 & 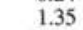 & 0.82 & 2. & 0.73 & 1.22 & 1.14 & 1.25 & 1.25 & 0.96 \\
\hline $\mathrm{Pb}$ & & & & & & & 6. & 2 & 4. & 75. & 39 & 6 & 14. & 8.4 & 2 & & & & & 7.5 & \\
\hline $\mathrm{Th}$ & 0.57 & 0.0 & 0.3 & 0.0 & 0. & 0. & 0.1 & 0.1 & 0.0 & 0.1 & 0. & 0.2 & 0.1 & 0.1 & 0.1 & 0.0 & 0.0 & 0.1 & 0.1 & 0.16 & 0.11 \\
\hline $\mathrm{U}$ & 0.58 & 0.07 & 0.16 & 0.03 & 0.03 & 0.02 & 0.07 & 0.04 & 0.04 & 0.13 & 0.09 & 0.11 & 0.09 & 0.07 & 0.12 & 0.09 & 0.05 & 0.08 & 0.18 & 0.18 & 0.05 \\
\hline
\end{tabular}


Table 2 (continued).

\begin{tabular}{|c|c|c|c|c|c|c|c|c|c|c|c|c|c|c|c|c|c|c|c|c|}
\hline Hole: & $837 \mathrm{~A}$ & $837 \mathrm{~A}$ & $837 \mathrm{~A}$ & $837 \mathrm{~A}$ & $837 \mathrm{~A}$ & $837 \mathrm{~A}$ & $838 \mathrm{~A}$ & $838 \mathrm{~A}$ & $838 \mathrm{~A}$ & $839 \mathrm{~A}$ & $839 \mathrm{~A}$ & $839 \mathrm{~A}$ & $839 \mathrm{~A}$ & $839 \mathrm{~A}$ & $839 \mathrm{~A}$ & $839 \mathrm{~A}$ & $839 \mathrm{~A}$ & $839 \mathrm{~A}$ & $839 \mathrm{~A}$ & $839 \mathrm{~A}$ \\
\hline Core, section: & $6 \mathrm{H}-4$ & $7 \mathrm{H}-4$ & $8 \mathrm{H}-1$ & $8 \mathrm{H}-2$ & $9 \mathrm{H}-1$ & $9 \mathrm{H}-3$ & $3 \mathrm{H}-2$ & $7 \mathrm{H}-2$ & $11 \mathrm{H}-5$ & $3 \mathrm{H}-3$ & $3 \mathrm{H}-4$ & $4 \mathrm{H}-4$ & $5 \mathrm{H}-2$ & $5 \mathrm{H}-3$ & $6 \mathrm{H}-5$ & $7 \mathrm{H}-2$ & $7 \mathrm{H}-6$ & $18 \mathrm{X}-\mathrm{CC}$ & $21 \mathrm{X}-\mathrm{CC}$ & $21 \mathrm{X}-\mathrm{CC}$ \\
\hline Interval $(\mathrm{cm})$ : & 132 & 33 & 108 & 49 & 53 & 93 & 147 & 89 & 30 & 144 & 22 & 35 & 15 & 137 & 136 & 90 & 125 & 6 & 33 & 33 \\
\hline Age (k.y.): & 1600 & 1700 & 1800 & 1825 & 2000 & 2050 & 750 & 1700 & 1900 & 700 & 750 & 920 & 1100 & 1150 & 1600 & 1700 & 1700 & 1700 & 1700 & 1700 \\
\hline Depth (mbsf): & 51.82 & 60.33 & 66.08 & 66.99 & 75.03 & 78.43 & 16.17 & 53.39 & 95.5 & 18.44 & 18.72 & 28.35 & 34.65 & 37.37 & 49.86 & 54.4 & 60.76 & 157.7 & 186.5 & 186.5 \\
\hline \multicolumn{21}{|c|}{ Major elements (wt\%): } \\
\hline $\mathrm{D}_{2}$ & & 51.68 & 52.52 & 52.20 & 51.51 & 50.79 & 51.55 & 52.91 & 54.93 & 51.23 & 50.56 & 52.82 & 53.71 & 53.32 & 52.16 & 50.13 & 52.58 & 52.52 & 51.03 & 51.41 \\
\hline $\mathrm{Al}_{2} \mathrm{O}_{2}$ & 14.69 & 15.10 & 14.79 & 14.93 & 14.41 & 14.34 & 14.80 & 13.94 & 13.77 & 14.93 & 14.10 & 13.41 & 13.13 & 14.60 & 14.71 & 14.46 & 14.40 & 14.40 & 14.04 & 14.07 \\
\hline F & 9.63 & 8.58 & 9.73 & 8.90 & 10.74 & 10.70 & 10.06 & 10.44 & 11.12 & 9.32 & 10.62 & 11.76 & 12.49 & 10.48 & & 10.14 & 9.64 & 10.21 & 9.99 & 9.83 \\
\hline$M$ & 5.96 & 6.97 & 5.88 & 6. & 5.17 & 6.85 & & 4.59 & 3.34 & 4.91 & 5.50 & 3.93 & 4.16 & .25 & 5.8 & 7 & 14 & 5.72 & 74 & 6.55 \\
\hline $\mathrm{C}_{\mathrm{i}}$ & 11.15 & 11.96 & 10.81 & 12.03 & 10.17 & 11.99 & 8.91 & 9.19 & 8.04 & 9.61 & 10.04 & 770 & 9.13 & .24 & 10. & 11.86 & 10.79 & 10.51 & 11.84 & 11.51 \\
\hline $\mathrm{Na} 2 \mathrm{O}$ & & & & & 1.56 & 1.25 & & 1.96 & & 2.25 & & & & 05 & & & 1.35 & & & 1.42 \\
\hline & 0.33 & 0.33 & 0.45 & 0.36 & & 0.28 & & 0.50 & & 0.51 & & 0.47 & 0.28 & 38 & & 0.24 & & & .28 & 0.34 \\
\hline & & & & 0.75 & 1.05 & & & 1.08 & & & & & & 39 & & & & & & 0.60 \\
\hline & 0.31 & 0.32 & 0.2 & 0.33 & 0.33 & 0.25 & & 0.36 & & & & & & & & & & & & 0.22 \\
\hline M & 0.18 & 0.17 & 0.16 & 0.19 & 0.23 & 0.20 & & 0.20 & & & & & & & & & & & & 0.20 \\
\hline & & & & & & & & & & & & & & & & & & & & 00 \\
\hline Total & 96.41 & 97.27 & 97.11 & 98.17 & 95.76 & 97.45 & 94.01 & 95.16 & 94.61 & 94.52 & 94.97 & 93.97 & 96.19 & 94.28 & 95.57 & 95.99 & 96.50 & 96.99 & 96.33 & 96.15 \\
\hline \multirow{2}{*}{\multicolumn{21}{|c|}{$\begin{array}{l}\text { Trace elements }(\mathrm{ppm}): \\
\mathrm{Rb}\end{array}$}} \\
\hline & 4.6 & 1.0 & 7.0 & 4.1 & 5.1 & 3.2 & 4.9 & 4.0 & 0.8 & 7.6 & 4.0 & 6.7 & 1.6 & 4.9 & 3.1 & 2.8 & 5.0 & 5.3 & 2.6 & 3.2 \\
\hline $\mathrm{Sr}_{\mathrm{r}}$ & 143 & 190 & 179 & 160 & 240 & 159 & 191 & 142 & 129 & 174 & 112 & 137 & 97 & 145 & 128 & & 129 & 154 & 127 & 143 \\
\hline 1 & 16.0 & 4.7 & 20.7 & 13.1 & 9.8 & 12.6 & 15.9 & 12.4 & 18.1 & 21.2 & 25.3 & 25.1 & 14.2 & 24.9 & 13.2 & 12.1 & 13.6 & 16.6 & 10.4 & 10.8 \\
\hline $\mathrm{Z}$ & 39 & 12 & 60 & 33 & 24 & 23 & 49 & 32 & 43 & 67 & 73 & 81 & 25 & & 29 & 26 & 32 & 44 & & 22 \\
\hline $\mathrm{N}$ & 0.46 & 0.15 & 0.88 & 0.44 & 0.25 & 0.09 & 0.62 & 0.39 & 0.11 & 0.94 & 1.07 & 1.16 & 0.12 & 1.22 & 0.25 & 0.08 & 0.58 & 0.63 & 0.12 & 0.26 \\
\hline D & 59 & 26 & 122 & 66 & 51 & $49^{\circ}$ & 56 & 57 & 78 & 65 & 38 & 75 & 89 & 57 & 60 & 41 & 67 & 83 & 43 & 54 \\
\hline L & 3.0 & 1.1 & 5.6 & 00 & 2.1 & 2.0 & 3.6 & 3.0 & 1.4 & 5.5 & 3.6 & 5. & 1.2 & 4.7 & 2 & 1. & 3.1 & 4. & 1.6 & 1.6 \\
\hline & . & 2 & 13 & & 5.1 & 5.0 & & 6.9 & & 12.0 & 9. & 12. & 2 & 11.6 & & & & & 7 & 4.4 \\
\hline & 6.4 & & & 6 & 4.0 & 5.2 & & 6.3 & & 9.2 & 8. & 10. & 3. & 10. & 5 & 4 & 5. & 7 & 3.6 & 4.0 \\
\hline & & & & 1 & 1. & i. & 2 & 1. & & 2 . & 2. & 2. & 1. & 2. & & 1. & 1.8 & 2 & 1. & 1.3 \\
\hline E & & & & & 0.44 & 0.62 & & & & 0.96 & 0.92 & 1.14 & 0.37 & 1.08 & & 0.56 & 0.61 & 0.71 & 0.35 & 0.41 \\
\hline & & & & & 0 & 0. & 0 & 0 . & 0 . & 0. & 0. & 0. & 0.3 & 0.7 & 0 & 0.3 & 4 & 0.4 & 0.3 & 0.3 \\
\hline & & & & 2. & 1. & 2. & 3. & 2. & 3. & 4. & 4. & 5. & 2. & 4. & 2. & 2. & 2. & 3. & 1. & 1.9 \\
\hline & & & & 0. & 0. & 0.48 & 0 & 0. & 0. & 0.93 & 0 . & 1. & 0. & 0.98 & 0 & 0.53 & 0.58 & 0.59 & 0.36 & 0.40 \\
\hline E & 1. & 0. & 2 . & 1. & 0.9 & 1.5 & 2. & 1. & 2. & 2. & 3. & 3 . & 1. & 3. & 2 . & & 1. & 1 & 1.2 & 1.2 \\
\hline L & 0.26 & 0.09 & 0.35 & 0.23 & 0.17 & 0.34 & 0. & 0. & & 0.43 & 0. & & 0 . & 0. & 0. & 0. & 0 & & 0.18 & 0.23 \\
\hline $\mathrm{Hf}$ & 1.22 & 0.39 & 1.51 & 0.76 & 0.65 & 1.51 & 1. & 0. & I. & 1. & 1. & 3. & & 2. & & 0.7 & 1. & 1. & 0.89 & 0.61 \\
\hline $\mathrm{Pb}$ & & & & & & & 6. & 5. & 4. & 3. & 3. & 14. & & 6 & & & 2.9 & 4 & 3 & 3.2 \\
\hline Th & 0.12 & 0.07 & 0.27 & 0.10 & 0.10 & 0.28 & 0.2 & 0.2 & 0.0 & 0.1 & 0.1 & 0.3 & 0.0 & 0.33 & 0.1 & 0.1 & 0.2 & 0.18 & 0.07 & 0.09 \\
\hline $\mathrm{U}$ & 0.06 & 0.03 & 0.13 & 0.08 & 0.06 & 0.36 & 0.08 & 0.08 & 0.04 & 0.13 & 0.04 & 0.12 & 0.01 & 0.11 & 0.08 & 0.06 & 0.13 & 0.15 & 0.05 & 0.04 \\
\hline
\end{tabular}


Table 3. Representative microprobe analyses of basaltic and basaltic andesite volcanic glass grains from the Tonga Platform (Site 840).

\begin{tabular}{|c|c|c|c|c|c|c|c|c|c|c|c|c|c|c|c|c|}
\hline Hole: & $840 \mathrm{~B}$ & $840 \mathrm{~A}$ & $840 \mathrm{~A}$ & $840 \mathrm{~A}$ & $840 \mathrm{C}$ & $840 \mathrm{C}$ & $840 \mathrm{C}$ & $840 \mathrm{C}$ & $840 \mathrm{C}$ & $840 \mathrm{C}$ & $840 \mathrm{~B}$ & $840 \mathrm{~B}$ & $840 \mathrm{C}$ & $840 \mathrm{C}$ & $840 \mathrm{~B}$ & $840 \mathrm{C}$ \\
\hline Core, section: & $1 \mathrm{X}-\mathrm{CC}$ & $1 \mathrm{H}-2$ & $1 \mathrm{H}-3$ & $1 \mathrm{H}-3$ & $1 \mathrm{H}-2$ & $1 \mathrm{H}-2$ & $1 \mathrm{H}-23$ & $2 \mathrm{H}-1$ & $3 \mathrm{H}-3$ & $4 \mathrm{H}-6$ & $12 X-5$ & $12 X-5$ & $9 \mathrm{H}-1$ & $10 \mathrm{H}-1$ & $24 \mathrm{X}-\mathrm{CC}$ & $13 \mathrm{H}-5$ \\
\hline Interval $(\mathrm{cm})$ : & 33 & 75 & 45 & 45 & 29 & 114 & 114 & 37 & 2 & 34 & 50 & 50 & 92 & 3 & 27 & 79 \\
\hline Age (k.y.): & 400 & 425 & 450 & 450 & 1700 & 1725 & 1725 & 1800 & 2700 & 3000 & 5000 & 5000 & 5250 & 5300 & 5330 & 5350 \\
\hline Depth (mbsf): & 1.02 & 2.25 & 3.50 & 3.50 & 39.79 & 40.64 & 40.64 & 47.87 & 59.88 & 74.34 & 110.50 & 1100.50 & 162.90 & 171.50 & 193.30 & 253.80 \\
\hline \multicolumn{17}{|c|}{ Major elements (wt\%): } \\
\hline $\mathrm{SiO}_{2}$ & 53.97 & 51.20 & 54.10 & 51.41 & 57.87 & 53.80 & 54.09 & 57.60 & 57.74 & 54.26 & 52.84 & 52.81 & 51.25 & 52.95 & 51.97 & 55.66 \\
\hline $\mathrm{Al}_{2} \mathrm{O}_{3}$ & 12.40 & 12.36 & 14.73 & 6.38 & 14.13 & 12.41 & 11.88 & 13.66 & 12.26 & 12.49 & 14.26 & 12.65 & 13.92 & 14.21 & 13.40 & 14.84 \\
\hline $\mathrm{Fe}_{2} \mathrm{O}_{3}$ & 13.64 & 15.89 & 12.24 & 18.10 & 9.70 & 13.85 & 14.53 & 10.11 & 11.69 & 16.28 & 10.97 & 12.67 & 12.08 & 11.81 & 12.65 & 10.25 \\
\hline $\mathrm{MgO}^{3}$ & 3.76 & 5.18 & 4.50 & 10.51 & 2.59 & 3.95 & 3.55 & 2.60 & 3.76 & 2.93 & 5.04 & 5.68 & 5.54 & 5.55 & 4.54 & 3.47 \\
\hline $\mathrm{CaO}$ & 8.66 & 11.10 & 9.21 & 7.45 & 7.67 & 8.65 & 8.58 & 7.36 & 9.61 & 8.82 & 9.35 & 9.16 & 10.87 & 9.41 & 9.37 & 8.31 \\
\hline $\mathrm{Na}_{2} \mathrm{O}$ & 1.62 & 1.66 & 1.99 & 1.27 & 1.46 & 1.87 & 1.97 & 1.31 & 1.10 & 1.93 & 2.46 & 1.62 & 2.25 & 1.71 & 1.73 & 1.78 \\
\hline $\mathrm{K}_{2} \mathrm{O}$ & 0.70 & 0.22 & 0.85 & 0.75 & 0.19 & 0.89 & 0.76 & 0.20 & 0.20 & 0.27 & 0.72 & 0.68 & 0.40 & 0.08 & 0.48 & 0.33 \\
\hline $\mathrm{TiO}_{2}$ & 1.28 & 1.28 & 0.96 & 0.88 & 1.04 & 1.28 & 1.31 & 1.07 & 0.90 & 1.27 & 1.00 & 1.08 & 1.09 & 0.95 & 1.20 & 1.04 \\
\hline $\mathrm{P}_{2} \mathrm{O}_{5}$ & 0.33 & 0.40 & 0.30 & 0.25 & 0.20 & 0.35 & 0.28 & 0.22 & 0.27 & 0.21 & 0.34 & 0.39 & 0.27 & 0.21 & 0.31 & 0.26 \\
\hline MnO & 0.28 & 0.31 & 0.27 & 0.36 & 0.22 & 0.28 & 0.29 & 0.23 & 0.19 & 0.26 & 0.22 & 0.28 & 0.25 & 0.27 & 0.21 & 0.24 \\
\hline Total & 96.63 & 99.60 & 99.14 & 97.34 & 95.07 & 97.33 & 97.22 & 94.34 & 97.71 & 98.72 & 97.19 & 97.03 & 97.94 & 97.16 & 95.86 & 96.15 \\
\hline \multicolumn{17}{|c|}{ Trace elements (ppm): } \\
\hline $\mathrm{Rb}$ & 6.7 & 2.8 & 2.8 & 7.0 & 1.4 & 0.0 & 0.0 & 0.1 & 0.0 & 1.3 & 4.1 & 10.0 & 2.8 & 1.3 & 1.0 & 2.2 \\
\hline $\mathrm{Sr}$ & 169 & 178 & 173 & $214^{\circ}$ & 150 & 174 & 176 & 153 & 126 & 152 & 212 & 264 & 183 & 148 & 232 & 126 \\
\hline $\mathrm{Y}$ & 20.4 & 10.6 & 13.7 & 14.4 & 20.0 & 18.8 & 17.4 & 17.1 & 10.0 & 17.0 & 16.9 & 17.5 & 20.4 & 18.5 & 14.8 & 18.7 \\
\hline $\mathrm{Zr}$ & 48 & 19 & 34 & 60 & 44 & 45 & 42 & 35 & 17 & 32 & 54 & 61 & 51 & 34 & 27 & 51 \\
\hline $\mathrm{Nb}$ & 0.33 & 0.00 & 0.14 & 0.58 & 0.17 & 0.08 & 0.08 & 0.37 & 0.01 & 0.02 & 0.52 & 0.55 & 0.50 & 0.06 & 0.12 & 0.22 \\
\hline $\mathrm{Ba}$ & 139 & 107 & 122 & 188 & 91 & 84 & 84 & 98 & 59 & 74 & 87 & 100 & 59 & 59 & 50 & 71 \\
\hline $\mathrm{La}$ & 3.8 & 2.0 & 2.1 & 3.9 & 1.5 & 1.4 & 1.1 & 2.4 & 0.4 & 1.5 & 3.3 & 4.6 & 2.1 & 0.8 & 1.9 & 1.7 \\
\hline $\mathrm{Ce}$ & 10.1 & 4.2 & 5.3 & 9.8 & 4.5 & 4.6 & 4.2 & 5.6 & 1.2 & 3.9 & 8.8 & 11.6 & 5.4 & 2.4 & 6.7 & 5.3 \\
\hline $\mathrm{Nd}$ & 8,3 & 3.1 & 3.8 & 6.1 & 5.0 & 47 & 4.1 & 3.9 & 2.2 & 4.1 & 7,4 & 9.2 & 5.7 & 3.6 & 6.9 & 5.6 \\
\hline $\mathrm{Sm}$ & 2.4 & 0.9 & 1.2 & 1.7 & 1.9 & 1.3 & 1.3 & 2.0 & 0.5 & 1.3 & 1.8 & 2.4 & 1.6 & 1.1 & 2.3 & 1.5 \\
\hline $\mathrm{Eu}$ & 0.94 & 0.11 & 0.23 & 0.11 & 0.81 & 0.29 & 0.27 & 0.57 & 0.14 & 0.57 & 0.78 & 0.64 & 0.48 & 0.35 & 0.79 & 0.76 \\
\hline $\mathrm{Tb}$ & 0.65 & 0.31 & 0.19 & 0.41 & 0.54 & 0.30 & 0.32 & 0.44 & 0.21 & 0.33 & 0.47 & 0.38 & 0.30 & 0.27 & 0.53 & 0.51 \\
\hline Dy & 3.8 & 1.7 & 1.7 & 1.8 & 3.4 & 2.6 & 2.5 & 2.9 & 1.6 & 2.2 & 3.6 & 2.6 & 2.3 & 1.8 & 3.1 & 3.0 \\
\hline Ho & 0.78 & 0.32 & 0.49 & 0.52 & 0.77 & 0.41 & 0.45 & 0.52 & 0.42 & 0.56 & 0.79 & 0.43 & 0.35 & 0.39 & 0.59 & 0.64 \\
\hline $\mathrm{Er}$ & 2.4 & 0.8 & 1.1 & 1.6 & 2.2 & 1.3 & 1.2 & 1.7 & 0.9 & 1.3 & 2.5 & 1.3 & 1.1 & 0.9 & 1.8 & 2.2 \\
\hline $\mathrm{Lu}$ & 0.34 & 0.10 & 0.19 & 0.18 & 0.38 & 0.19 & 0.17 & 0.43 & 0.19 & 0.26 & 0.47 & 0.23 & 0.12 & 0.13 & 0.30 & 0.28 \\
\hline $\mathrm{Hf}$ & 1.51 & - & - & - & 1.40 & - & - & - & - & 0.95 & 1.98 & 1.04 & 0.44 & 0.22 & 0.81 & 1.17 \\
\hline $\mathrm{Th}$ & 0.16 & 0.06 & 0.09 & 0.17 & 0.10 & 0.07 & 0.05 & 0.07 & 0.03 & 0.04 & 0.17 & 0.14 & 0.05 & 0.01 & 0.05 & 0.02 \\
\hline $\mathrm{U}$ & 0.09 & 0.01 & 0.09 & 0.23 & 0.09 & 0.07 & 0.00 & 0.10 & 0.01 & 0.03 & 0.15 & 0.11 & 0.03 & 0.00 & 0.05 & 0.03 \\
\hline
\end{tabular}

projected back to a volatile-free composition, gives approximately $82 \% \mathrm{SiO}_{2}$. Allowing for sodium enhancement of some $5 \%$, a more reasonable anhydrous rhyolitic glass figure results. Rather than compute a sodium-loss correction derived from Figure 4 and then correct all the resultant data to a volatile-free basis, the data have been left uncorrected. They have instead been weeded for suspiciously low totalling analyses below the lower line on Figure 3, which takes account of the fact that volatiles increase with silica and uses Burnham and Jahn's (1962) estimates of the water contents of saturated basaltic and andesitic glasses.

It should be noted that the trace element data obtained on the ion microprobe are not affected by effects analogous to sodium loss under the electron beam, though a correction would still be necessary to arrive at volatile-free values.

The basic test of the robustness of the data is the extent of preservation of magmatic interelement ratios. It will be argued below that not only do immobile elements behave in a coherent fashion but that in basic glasses at least, incompatible mobile elements such as Ba and even $\mathrm{Rb}$ are relatively enriched in step with equivalently incompatible immobile elements.

\section{General Compositional Variations across the Lau Basin}

Figure 5 shows the variation in total silica contents of probed glasses from across the Lau Basin and plots their variation through time. Figure 6 shows the variation in silica contents of glass grains on the Tonga Platform at Site 840 with time. The main finding within the Lau Basin is that the principal differences between sites are in the distributions of silica contents. Some sites are dominated by a bimodal suite of glasses, others by intermediate, andesitic compositions. The bulk of the analyzed glasses fall on different segments of a similar island arc tholeiite fractionation trend. Within this pattern of major element homogeneity there is a range of levels of incompatible element enrichment. We hope to demonstrate that this shows a systematic trend with time at Site 840 , for which we have most data, but it is not yet clear that any features of the geochemistry are attributable to parental magma composition or fractionation path, which are sufficiently distinctive between sites to act as a source discriminant.

It is noteworthy that at each site little change appears in the range of the major element compositions of glasses being deposited from the top to the bottom of any one particular hole. However, differences in the composition of the sands do appear across the basin. Sites 837 and 839, which lie closest to the active Eastern Lau Spreading Center (ELSC) show approximate bimodal populations, dominated by basaltic andesite and rhyolite. An apparent gap in silica contents is present between $65 \%$ and $75 \% \mathrm{SiO}_{2}$. A bimodal pattern is also recognized from the glasses at Site 840 younger than $5.0 \mathrm{Ma}$ (Fig. 5), but this pattern differs in having lower silica contents in the high silica range. High silica glasses at Sites 837 and 839 range as high as $78 \% \mathrm{SiO}_{2}$, compared with a maximum of approximately $76 \% \mathrm{SiO}_{2}$ at Site 840 (Fig. 6). Site 840, on the other hand, has experienced an earlier history, before basin rifting, in which a complete spectrum of silica values was produced. The bias to andesitic values is characteristic of a different phase of volcanic activity (i.e., from 7.0 to $5.0 \mathrm{Ma}$ ) and is a feature shared by sites from the western Lau Basin (Sites 834 and 835 ). These form a separate chemical grouping to the sub-basins of the central Lau Basin. Finally, Site 836 is unusual in that an almost unimodal sand composition of basaltic andesites is present, much of which is in the form of very proximal volcanic breccias and hyaloclastites. Although this may seem to be in keeping with the position of Site 836 within basaltic crust produced at an oceanic spreading center, it is noteworthy that igneous basement rocks generally yield analyses with a consistently lower silica content $\left(48 \%-52 \% \mathrm{SiO}_{2}\right.$; Parson, Hawkins, Allan, et al., 1992, pp. 257-259).

Figure 7 shows plots of potassium content vs. total silica for individual sites. The diagram is divided into fields after the classification of Peccerillo and Taylor (1976). The plots clearly show that the vast majority of the analyzed glasses lie within the low-K tholeiite field, with a minor overlap into the medium-K field. A number of important exceptions to note include the presence of a small quantity 
Table 3 (continued).

\begin{tabular}{|c|c|c|c|c|c|c|c|c|c|c|c|c|c|c|c|c|c|}
\hline Hole: & $840 \mathrm{~B}$ & $840 B$ & $840 \mathrm{~B}$ & $840 \mathrm{~B}$ & $840 \mathrm{~B}$ & $840 B$ & $840 \mathrm{~B}$ & $840 \mathrm{~B}$ & $840 \mathrm{~B}$ & $840 \mathrm{~B}$ & $840 \mathrm{~B}$ & $840 \mathrm{~B}$ & $840 \mathrm{~B}$ & $840 \mathrm{~B}$ & $840 \mathrm{~B}$ & $840 \mathrm{~B}$ & $840 \mathrm{~B}$ \\
\hline Core, section: & $30 \mathrm{X}-1$ & $33 \mathrm{X}-1$ & $33 \mathrm{X}-1$ & $34 X-1$ & $35 X-3$ & $38 \mathrm{X}-1$ & $40 X-1$ & $40 X-1$ & $41 X-2$ & $41 X-2$ & $42 X-3$ & $48 \mathrm{X}-1$ & $50 X-1$ & $52 X-2$ & $56 X-4$ & $57 X-3$ & $62 X-5$ \\
\hline Interval $(\mathrm{cm})$ : & 12 & 21 & 21 & 86 & 22 & 17 & 121 & 121 & 39 & 39 & 3 & 99 & 134 & 5 & 108 & 81 & 42 \\
\hline Age (k.y.): & 5400 & 5650 & 5650 & 5800 & 5850 & 5950 & 6000 & 6000 & 6050 & 6050 & 6100 & 6400 & 6475 & 6525 & 6750 & 6800 & 7000 \\
\hline Depth (mbsf): & 279.20 & 308.40 & 308.40 & 318.70 & 330.70 & 347.30 & 367.30 & 367.31 & 377.59 & 377.59 & 388.83 & 443.79 & 463.44 & 482.95 & 525.67 & 533.35 & 584.42 \\
\hline \multicolumn{18}{|c|}{ Major elements (wt\%): } \\
\hline $\mathrm{SiO}_{2}$ & 54.97 & 54.23 & 52.57 & 50.39 & 50.90 & 50.05 & 53.94 & 53.57 & 53.61 & 55.78 & 52.83 & 53.72 & 53.87 & 54.76 & 53.83 & 52.39 & 50.96 \\
\hline $\mathrm{Al}_{2} \mathrm{O}_{3}$ & 12.55 & 14.23 & 13.70 & 13.94 & 12.33 & 13.23 & 13.02 & 13.23 & 14.78 & 14.78 & 13.64 & 14.57 & 14.56 & 12.83 & 13.91 & 13.58 & 12.57 \\
\hline $\mathrm{Fe}_{2} \mathrm{O}_{3}$ & 13.05 & 11.46 & 12.03 & 10.66 & 15.64 & 12.39 & 12.56 & 12.74 & 11.40 & 11.19 & 12.55 & 11.11 & 10.56 & 11.23 & 11.67 & 12.01 & 13.18 \\
\hline $\mathrm{MgO}^{2}$ & 3.36 & 2.76 & 3.71 & 6.57 & 4.77 & 6.11 & 3.69 & 4.42 & 4.01 & 3.82 & 3.56 & 3.50 & 3.49 & 4.94 & 3.56 & 3.70 & 4.96 \\
\hline $\mathrm{CaO}$ & 7.91 & 7.24 & 8.32 & 11.78 & 9.68 & 12.15 & 7.93 & 9.22 & 8.73 & 8.46 & 8.21 & 7.84 & 7.86 & 7.38 & 8.27 & 8.28 & 10.41 \\
\hline $\mathrm{Na}_{2} \mathrm{O}$ & 2.07 & 1.94 & 2.05 & 1.63 & 1.97 & 1.82 & 2.15 & 2.04 & 2.11 & 1.84 & 1.77 & 2.04 & 2.09 & 1.82 & 1.56 & 2.13 & 1.45 \\
\hline $\mathrm{K}, \mathrm{O}$ & 0.59 & 0.47 & 0.47 & 0.29 & 0.37 & 0.45 & 0.43 & 0.27 & 0.33 & 0.32 & 0.42 & 0.64 & 0.50 & 0.57 & 0.33 & 0.36 & 0.31 \\
\hline $\mathrm{TiO}_{2}$ & 1.56 & 1.27 & 1.45 & 0.92 & 1.30 & 0.97 & 1.38 & 1.11 & 1.17 & 1.26 & 1.37 & 1.41 & 1.24 & 1.13 & 1.33 & 1.33 & 1.00 \\
\hline $\mathrm{P}_{2} \mathrm{O}_{5}^{2}$ & 0.39 & 0.29 & 0.35 & 0.31 & 0.24 & 0.34 & 0.34 & 0.22 & 0.31 & 0.29 & 0.32 & 0.35 & 0.39 & 0.37 & 0.26 & 0.28 & 0.19 \\
\hline $\mathrm{MnO}$ & 0.23 & 0.23 & 0.26 & 0.22 & 0.32 & 0.27 & 0.24 & 0.26 & 0.22 & 0.20 & 0.21 & 0.26 & 0.21 & 0.26 & 0.19 & 0.21 & 0.30 \\
\hline Total & 96.70 & 94.11 & 94.91 & 96.70 & 97.51 & 97.78 & 95.68 & 97.07 & 96.66 & 97.94 & 94.88 & 95.44 & 94.76 & 95.29 & 94.91 & 94.27 & 95.33 \\
\hline \multicolumn{18}{|c|}{ Trace elements (ppm): } \\
\hline $\mathrm{Rb}$ & 3.8 & 4.0 & 4.5 & 2.0 & 4.2 & 2.3 & 2.9 & 2.8 & 2.8 & 2.0 & 3.2 & 5.9 & 3.9 & 5.6 & 2.4 & 2.1 & 6.9 \\
\hline $\mathrm{Sr}$ & 211 & 158 & 142 & 152 & 189 & 159 & 160 & 158 & 156 & 152 & 159 & 209 & 222 & 186 & 140 & 136 & 116 \\
\hline $\mathrm{Y}$ & 24.2 & 23.4 & 22.4 & 14.0 & 20.1 & 19.5 & 21.4 & 21.1 & 23.0 & 22.2 & 25.2 & 23.5 & 21.3 & 21.3 & 24.7 & 24.3 & 23.6 \\
\hline $\mathrm{Zr}$ & 89 & 92 & 86 & 39 & 57 & 63 & 66 & 65 & 74 & 70 & 80 & 79 & 67 & 87 & 61 & 60 & 104 \\
\hline $\mathrm{Nb}$ & 1.11 & 1.09 & 0.88 & 0.55 & 0.69 & 0.54 & 0.64 & 0.63 & 0.63 & 0.54 & 0.70 & 1.14 & 0.85 & 1.37 & 0.33 & 0.31 & 1.81 \\
\hline $\mathrm{Ba}$ & 118 & 97 & 90 & 52 & 78 & 67 & 75 & 74 & 83 & 80 & 80 & 103 & 83 & 101 & 67 & 66 & 138 \\
\hline $\mathrm{La}$ & 5.2 & 4.7 & 4.2 & 2.3 & 3.0 & 2.4 & 3.0 & 3.0 & 3.0 & 2.8 & 3.6 & 5.3 & 4.5 & 5.8 & 2.3 & 2.2 & 5.7 \\
\hline $\mathrm{Ce}$ & 13.5 & 11.4 & 10.8 & 5.7 & 8.4 & 6.9 & 8.3 & 8.2 & 8.6 & 8.5 & 9.9 & 14.2 & 12.4 & 13.4 & 7.2 & 6.8 & 15.6 \\
\hline $\mathrm{Nd}$ & 10.0 & 10.2 & 9.7 & 4.4 & 7.1 & 6.6 & 7.8 & 7.7 & 8.1 & 7.2 & 8.0 & 11.7 & 10.5 & 10.6 & 7.2 & 6.9 & 11.3 \\
\hline $\mathrm{Sm}$ & 2.9 & 2.5 & 3.0 & 1.4 & 2.1 & 2.0 & 2.1 & 2.0 & 2.4 & 2.3 & 2.6 & 2.9 & 2.9 & 3.1 & 2.5 & 2.1 & 3.1 \\
\hline $\mathrm{Eu}$ & 0.85 & 1.16 & 1.11 & 0.51 & 0.83 & 0.69 & 0.80 & 0.79 & 1.05 & 0.81 & 0.84 & 1.26 & 1.10 & 1.25 & 0.84 & 0.76 & 0.92 \\
\hline $\mathrm{Tb}$ & 0.56 & 0.75 & 0.68 & 0.30 & 0.56 & 0.43 & 0.43 & 0.42 & 0.68 & 0.55 & 0.61 & 0.70 & 0.60 & 0.68 & 0.61 & 0.53 & 0.59 \\
\hline Dy & 3.3 & 5.0 & 4.6 & 2.1 & 3.7 & 3.3 & 3.2 & 3.1 & 4.6 & 3.5 & 4.8 & 4.7 & 3.9 & 4.5 & 4.2 & 3.9 & 3.8 \\
\hline Ho & 0.72 & 1.03 & 0.94 & 0.41 & 0.76 & 0.62 & 0.65 & 0.64 & 1.00 & 0.86 & 0.93 & 0.89 & 0.77 & 0.93 & 0.77 & 0.80 & 0.80 \\
\hline $\mathrm{Er}$ & 2.0 & 3.3 & 3.2 & 1.2 & 2.0 & 1.9 & 1.9 & 1.9 & 2.9 & 2.5 & 2.4 & 2.6 & 1.9 & 3.0 & 2.6 & 2.1 & 2.1 \\
\hline $\mathrm{Lu}$ & 0.31 & 0.47 & 0.54 & 0.18 & 0.40 & 0.31 & 0.31 & 0.31 & 0.49 & 0.44 & 0.49 & 0.41 & 0.35 & 0.56 & 0.33 & 0.39 & 0.36 \\
\hline Hf & 1.50 & 2.67 & 2.27 & 0.79 & 2.10 & 1.19 & 1.12 & 1.11 & 2.35 & 2.01 & 2.42 & 2.00 & 1.49 & 3.02 & 1.38 & 0.82 & 1.93 \\
\hline Th & 0.12 & 0.36 & 0.24 & 0.12 & 0.13 & 0.05 & 0.03 & 0.03 & 0.10 & 0.17 & 0.02 & 0.19 & 0.18 & 0.41 & 0.07 & 0.06 & 0.13 \\
\hline U & 0.08 & 0.17 & 0.15 & 0.05 & 0.10 & 0.05 & 0.02 & 0.02 & 0.06 & 0.17 & 0.10 & 0.12 & 0.08 & 0.18 & 0.04 & 0.04 & 0.08 \\
\hline
\end{tabular}

of medium- and high-K glass at Sites 834 and 835 , as well as a spread of higher potassium values at Site 840 . Although a great number of medium-K glasses are present, a scatter of points into the high- $\mathrm{K}$ and shoshonitic fields is also observed. The time distribution of alkali-rich glasses at Site 840 is described by Figure 8 , which plots the ratio of total alkali contents relative to silica against time. This clearly shows that, although these have been restricted in range and magnitude since approximately $5.0 \mathrm{Ma}$, the earlier history is characterized by a minor component of more alkaline volcanism. In all these cases, the total alkali contents analyzed will be lower than the true value because of the ease with which these elements volatilize under the beam during analysis, and some uncertainty must also be attached to the high values.

The AFM (alkali/iron/magnesium) diagram (Fig. 9) for Lau Basin glasses show typical tholeiitic trends. The alkali loss inevitably disperses the data and disguises the tightness of the iron-enrichment trends. This is illustrated better on Figures 10A and 10B, which show $\mathrm{Fe}_{2} \mathrm{O}_{3}$ and $\mathrm{TiO}_{2}$ vs. silica for all the weeded data. An extreme tholeiitic iron-enrichment trend for the Krafla volcanic series in Iceland is shown for comparison. The apices of the trends, attributable to the onset of Fe-Ti oxide precipitation, occurs at significantly higher $\mathrm{SiO}_{2}$ in the arc suites, $54 \%$, compared to $51 \%$ in Iceland. On these amalgamated plots, the peak level of iron enrichment at Site 840 (Tonga Platform) appears to be greater than at the Lau Basin sites.

A single grain that does not fall into the tholeiitic trend is noted at Site 834 from the base of the upper Pliocene (Sample 135-834A-7H-4, $33 \mathrm{~cm}$; Table 1).

The major element analyses obtained from Tonga Platform glass grains at Site 840 broadly confirm the conclusions of Cawood (1991) in showing a generally consistent tholeiitic magmatic history right from the upper Miocene to the present, without noticeable disturbance at the time of rifting of the Lau Basin. The analyses from Site 840, however, extend his findings in suggesting an earlier period of volcanism (7.0-5.0 Ma; late Miocene), in which a broad spectrum of total silica contents was erupted, as well as minor volumes of more alkalic material. This contrasts with the low-K, bimodal volcanism recorded since shortly after basin rifting. Similar major element contents to these post-5.0 Ma glasses at Site 840 are found in the sediments cored from the Lau Basin (Sites 834-839), where almost all the glass is also of low-K tholeiitic character. However, the glasses of the central and eastern Lau Basin (Sites 837 and 839) show a bimodal silica pattern. This is not the same as the bimodal distribution in the PliocenePleistocene at Site 840 . No trace of the Miocene andesitic trend seen at Site 840 exists within the sediments of the central Lau Basin.

Despite the generally indistinguishable parentage of these suites, the differences between the sites in silica distribution over comparable time periods must reflect the behavior of individual volcanic edifices, with distinct patterns of replenishment, fractionation, and eruption. For example, the contrast in silica contents between the ashes at Sites 834 and 835 , at Sites $837-839$, and at Site 840 indicate a separate origin for the sediments among each of these groups. This chemical grouping is consistent with the sedimentary facies information pointing to their proximal derivation within the backarc basin in the case of Sites 834-839, or directly from arc to forearc at Site 840. Given that the sediments at each backarc site form proximal deposits around submarine volcanoes active during a period of no known arc volcanism on the eastern side of the basin, the volcaniclastic record in the Lau Basin indicates a change in the character of the volcanism from andesitic along a subaerial arc volcanic front to bimodal on a migrating front of submarine intrabasinal seamount volcanoes. The change in character does not occur immediately at the point of basin rifting but can be placed between the time of supply for the youngest site showing the older silica distribution (Site 835; an andesitic suite) at 3.25 Ma and the oldest site showing the newer bimodal distribution (Site 837 ) at $2.70 \mathrm{Ma}$ (i.e., at approximately $3.0 \mathrm{Ma}$, the middle Pliocene). Interestingly, this date approximately coincides with the age of initiation of the Tofua Arc inferred from the ash record at Site 840 (Tappin et al., this volume).

An exception to the general pattern is Site 836. The dominance of coarse basaltic andesite debris at Site 836, and the absence of significant acidic debris is consistent with this site's position firmly within 

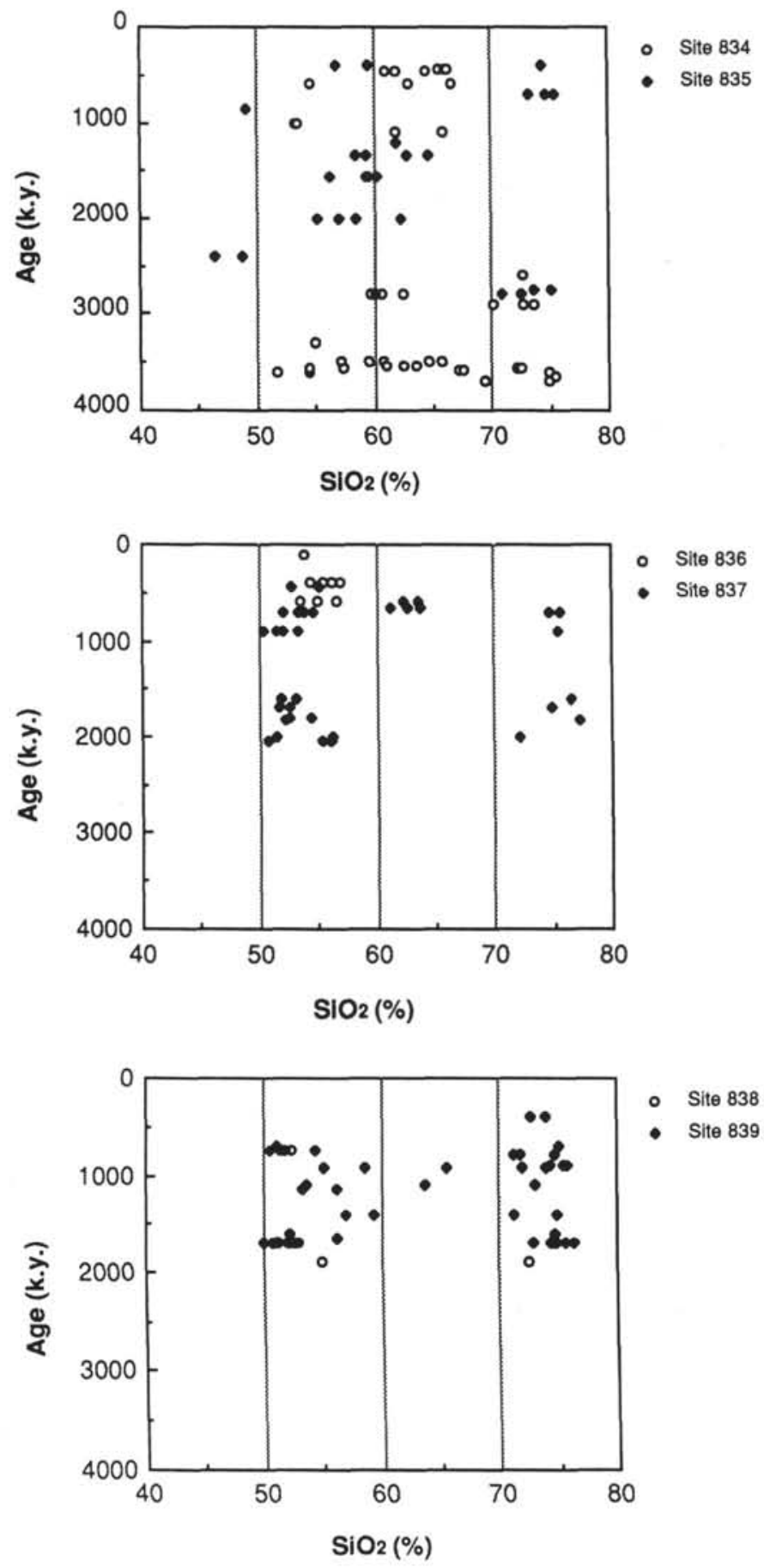

Figure 5. Diagram showing the variation in total silica contents of volcanic glass grains with age for Sites 834-839 (Lau Basin). Silica contents plotted are contents as analyzed.

basaltic crust generated at a backarc spreading center. This implies that the volcaniclastic debris is from the exposed basement. The young age $(600$ k.y.) and position place this site away from any potential influence from intrabasinal seamount volcanoes or the Lau Ridge. It is noteworthy that although the total silica values recorded at Site 836 are biased towards basaltic andesite values, the major element compositions of these glasses are effectively identical to basaltic andesite glasses from the other Lau Basin Sites. The singular nature of the Site 836 sediments must be the result of a combination of the tectonic regime in which the sub-basin was generated, coupled

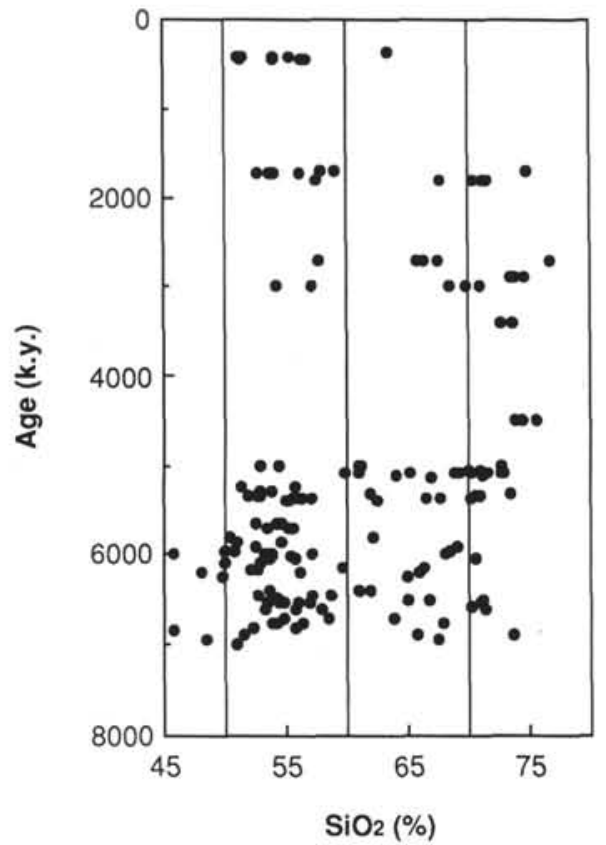

Figure 6. Diagram showing the variation in total silica contents of volcanic glass grains with age for Site 840 (Tonga Platform).

with the site's remoteness from potential source volcanoes in the attenuated arc crust of the western Lau Basin.

That some reworking of the Lau Ridge did occur is possibly suggested by the presence of a single "calc-alkaline" glass grain at Site 834 (i.e., one showing no iron enrichment at intermediate silica values; see Table 1). This might reflect the influence of the lowerupper Miocene Lau Volcanic Group of the Lau Ridge, which is known to be partially of calc-alkaline character (Cole et al., 1985). Higher up the section, the small number of more alkaline glasses found at both Sites 834 and 835 mirror the sporadic Pleistocene alkalic volcanism of the Mago Volcanic Group, also known from the Lau Ridge.

\section{Pyroxenes}

A small number of pyroxenes were analyzed and a representative set of results are presented in Table 4 and plotted on the pyroxene quadrilateral in Figure 11. To maximize confidence in the results, only those pyroxenes with analytical totals greater than $98 \%$ are included for consideration. Sites 836 and 838 have only one analysis each, both hypersthenes. However, at Sites 834, 837, and 839, where a greater number of pyroxenes have been analyzed, hypersthene is also accompanied by a number of diopside and augite grains. Pyroxenes from Site 835 form a separate group, all being tightly grouped in the salite-diopside field. Furthermore, they differ from those of the other sites in having significant $\mathrm{Al}$ contents, but low $\mathrm{Na}$ and $\mathrm{Ti}$, reflecting Tschermak's substitution, a feature common to pyroxenes from silicaundersaturated igneous rocks (Le Bas, 1962). The clinopyroxene analyses were plotted on a silica/alumina diagram (Fig. 12; Le Bas, 1962), allowing their further discrimination. This plot suggests that although most of the clinopyroxenes were crystallized from a subalkalic magma, a number of others, generally derived from the lower parts of the Site 840 section and Site 835 , would seem to be the result of fractionation from subalkaline or peralkaline liquids.

The two-pyroxene-assemblage recorded from both the Lau Basin and Tonga Platform is typical of a low-K tholeiite fractionation trend (Ewart, 1982) and of the pyroxene assemblage in modern arc tholeiites from the Tofua Arc (Ewart et al., 1973), although it must be recognized that these are all separate grain analyses rather than coexisting phases in a single rock. Nevertheless, the chemistry of the pyroxenes from the 

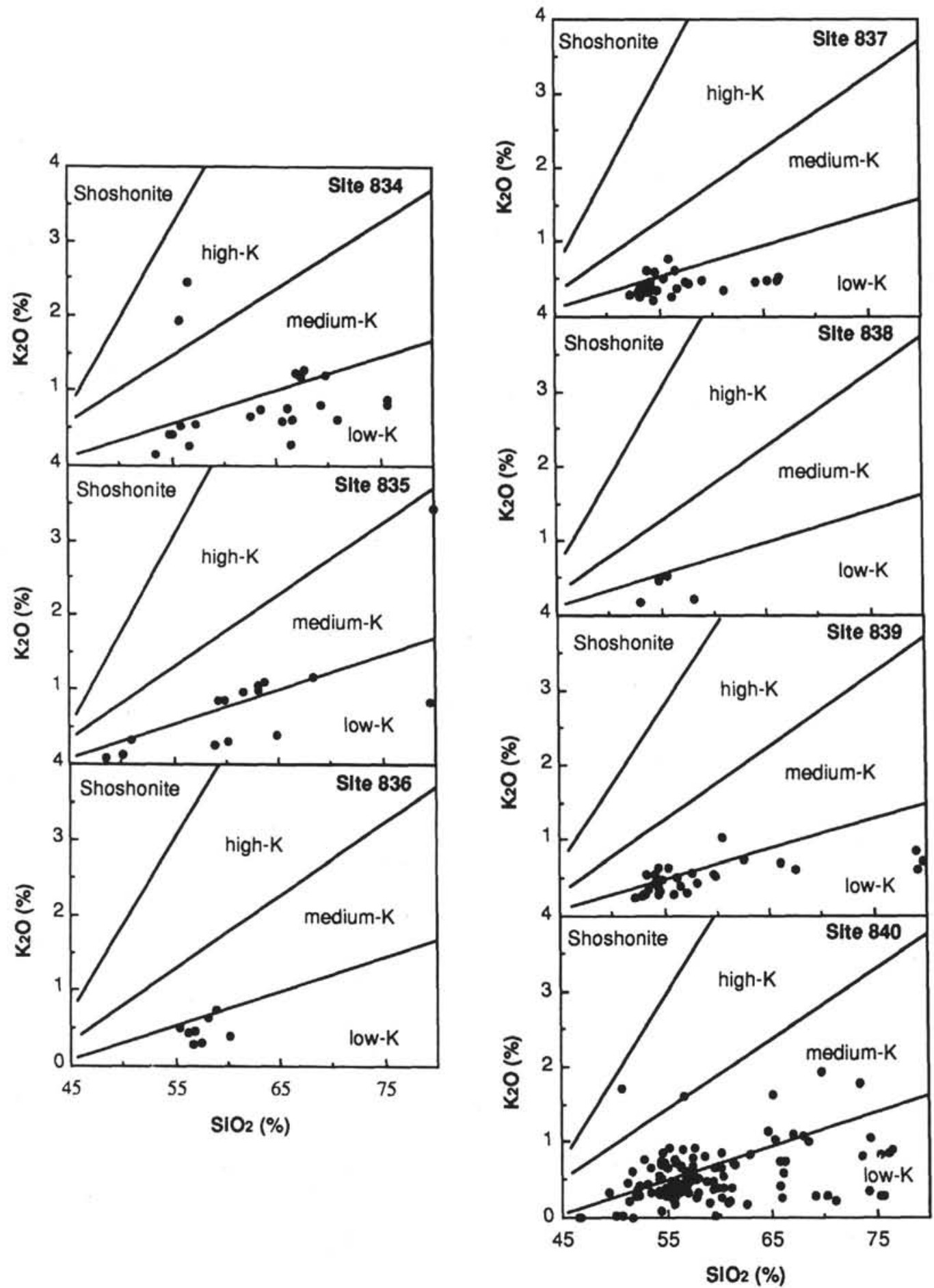

Figure 7. Compositional plots of $\mathrm{K}_{2} \mathrm{O}$ vs. $\mathrm{SiO}_{2}$ for detrital glass from Sites 834 to 840 , showing the dominance of low-K tholeiite, although with greater spread into high-K compositions at Site 840. Compositional fields are from Peccerillo and Taylor (1976).

Lau Basin is compatible with their derivation from tholeiitic volcanoes. An earlier history of more alkalic volcanism is suggested by the presence of relatively alumina-rich clinopyroxenes in the Miocene of Site 840 , which could be associated with the glasses with higher total alkalies to silica, from the Miocene part of the section. Such pyroxenes are noticeably absent from the younger sediments of the Lau Basin itself. Clinopyroxenes with an inferred alkaline source are found, however, at Site 835 and suggest their derivation from a nearby source. Since Site 835 is believed to lie within attenuated arc crust, these alkali pyroxenes are by inference derived by reworking of the older arc basement during sub-basin rifting.

The division of the Lau Basin volcanic history into two periods on the basis of the glass shard major element data is thus supported by the pyroxene data from Site 835 . The earlier period's eruptive products are 
Table 4. Representative microprobe analyses of pyroxenes from each of the Lau Basin and Tonga Platform sites (834-840).

\begin{tabular}{|c|c|c|c|c|c|c|c|c|c|c|c|c|c|c|c|}
\hline Hole: & $834 \mathrm{~A}$ & $834 \mathrm{~A}$ & $834 \mathrm{~A}$ & $834 \mathrm{~A}$ & $834 \mathrm{~A}$ & $835 \mathrm{~A}$ & $835 \mathrm{~A}$ & $835 \mathrm{~A}$ & $836 \mathrm{~A}$ & $837 \mathrm{~A}$ & $837 \mathrm{~A}$ & 837A & $837 \mathrm{~A}$ & $838 \mathrm{~A}$ & $839 \mathrm{~A}$ \\
\hline Core, section: & $1 \mathrm{H}-2$ & $3 \mathrm{H}-1$ & $3 \mathrm{H}-4$ & $6 \mathrm{H}-2$ & $7 \mathrm{H}-4$ & $4 \mathrm{H}-5$ & $4 \mathrm{H}-5$ & $4 \mathrm{H}-5$ & $1 \mathrm{H}-1$ & $2 \mathrm{H}-4$ & $4 \mathrm{H}-6$ & $6 \mathrm{H}-4$ & $8 \mathrm{H}-2$ & $11 X-4$ & $3 \mathrm{H}-3$ \\
\hline Interval $(\mathrm{cm})$ : & 37 & 72 & 40 & 35 & 78 & 57 & 57 & 109 & 84 & 103 & 67 & 132 & 49 & 30 & 144 \\
\hline Age (k.y.): & 600 & 1200 & 1250 & 2800 & 3300 & 900 & 900 & 950 & 100 & 550 & 900 & 1500 & 1900 & 1900 & 800 \\
\hline Depth (mbsf): & 1.87 & 17.82 & 22.00 & 47.45 & 60.38 & 35.07 & 35.07 & 35.59 & 0.84 & 13.53 & 35.17 & 51.82 & 66.99 & 94.00 & 18.44 \\
\hline \multicolumn{16}{|c|}{ Major elements (wt\%): } \\
\hline $\mathrm{SiO}_{2}$ & 51.48 & 52.13 & 51.22 & 49.19 & 52.54 & 51.11 & 49.76 & 47.76 & 52.36 & 51.11 & 51.98 & 52.51 & 51.28 & 50.02 & 49.73 \\
\hline $\mathrm{TiO}_{2}^{2}$ & 0.21 & 0.12 & 0.18 & 0.19 & 0.18 & 0.37 & 0.33 & 1.05 & 0.13 & 0.30 & 0.33 & 0.18 & 0.30 & 0.23 & 0.62 \\
\hline $\mathrm{Al}_{2} \mathrm{O}_{3}$ & 1.07 & 1.32 & 1.28 & 0.47 & 1.21 & 3.31 & 4.21 & 6.49 & 1.55 & 1.22 & 1.26 & 0.51 & 1.21 & 0.54 & 4.08 \\
\hline $\mathrm{Fe}_{2}^{2} \mathrm{O}_{3}$ & 22.38 & 15.02 & 11.06 & 30.92 & 3.15 & 4.09 & 5.45 & 6.62 & 16.77 & 15.31 & 10.29 & 23.49 & 15.29 & 28.46 & 7.46 \\
\hline $\mathrm{MnO}$ & 0.74 & 0.44 & 0.70 & 1.41 & 0.10 & 0.11 & 0.14 & 0.18 & 0.37 & 0.59 & 0.47 & 1.09 & 0.56 & 1.56 & 0.18 \\
\hline $\mathrm{MgO}$ & 21.22 & 23.89 & 13.91 & 12.61 & 17.12 & 16.92 & 15.80 & 15.21 & 24.89 & 12.30 & 13.48 & 19.97 & 11.98 & 15.83 & 15.88 \\
\hline $\mathrm{CaO}$ & .00 & 5.07 & 20.46 & 4.14 & 23.87 & 21.12 & 21.32 & 19.74 & 2.14 & 18.46 & 21.01 & 1.35 & 19.32 & 1.66 & 19.44 \\
\hline $\mathrm{Na}_{2} \mathrm{O}$ & 0.01 & 0.04 & 0.21 & 0.03 & 0.18 & 0.13 & 0.17 & 0.24 & 0.14 & 0.16 & 0.25 & 0.02 & 0.14 & 0.04 & 0.26 \\
\hline $\mathrm{K}_{2} \tilde{\mathrm{O}}$ & 0.01 & 0.01 & 0.01 & 0.01 & 0.00 & 0.01 & 0.00 & 0.01 & 0.02 & 0.01 & 0.00 & 0.00 & 0.01 & 0.00 & 0.01 \\
\hline $\mathrm{P}_{2} \mathrm{O}_{5}$ & 0.04 & 0.09 & 0.32 & 0.07 & 0.40 & 0.26 & 0.48 & 0.29 & 0.03 & 0.24 & 0.36 & 0.03 & 0.30 & 0.04 & 0.35 \\
\hline Total & 99.35 & 98.24 & 99.53 & 99.21 & 98.93 & 98.52 & 98.25 & 98.63 & 98.51 & 100.00 & 99.76 & 99.32 & 100.66 & 98.61 & 98.64 \\
\hline
\end{tabular}

dominantly andesitic in character, but include locally more alkaline magmas. Whether the latter are eruptions from the andesitic centers themselves or are from separate centers altogether is not clear.

\section{Feldspars}

Analyzed feldspars are all plagioclase. The compositions are displayed in Table 5 and plotted in Figure 13, using only crystals that have analytical totals greater than $98 \%$. The Ca-rich feldspars are the most common variety found, principally bytownite $(A n=70 \%-90 \%)$, which is comparable with the bytownite compositions (An $=80 \%$ 90\%) reported by Ewart et al. (1973) for the Holocene volcanics of the Tonga Islands. At Site 834, the feldspars show a tight grouping of values around $80 \%-90 \%$ anorthite; however, elsewhere a greater spread of values down to less than $50 \%$ anorthite is noted. Site 838 forms an exception to the general pattern, as the feldspars analyzed here form a tight group of approximately $\mathrm{An}=50 \%$ (i.e., andesine and labradorite), although the small number involved makes the statistical significance of this doubtful.

\section{TRACE AND RARE EARTH ELEMENT CHEMISTRY}

The results of trace and rare earth element analyses performed using the ion microprobe on selected low silica volcanic glass grains are shown in Tables 2 and 3. Particular attention was given to grains from Sites $834,835,837,839$ and 840 to achieve both a coverage of trace element variation across the basin and at individual sites with time. A smaller number of analyses were also performed on material from Sites 836 and 838 .

The precision and reproducibility of the trace element measurements are assumed to closely approximate to those given by the Poisson counting statistics for each element. This assumption is supported by earlier work on the same probe (e.g., Hinton and Upton, 1991). In particular, the straight nature of the REE plots obtained from the glasses suggest that the errors are not significantly greater than the uncertainties in the counting statistics, even at low concentrations $(<1.0$ ppm). Chemically similar elements are seen to show the same trends within the data set, regardless of whether the element is a relatively abundant one (e.g., $\mathrm{Zr}$ ) or present in low concentrations (e.g., $\mathrm{Nb}$ ). Table 6 shows the count rate errors for a typical analysis from Site 840 . It is noteworthy that for some geochemically important elements, such as $\mathrm{Nb}$, the ion probe method is able to achieve good precision $(<5 \%$ error) when concentrations are very low $(<1 \mathrm{ppm})$, as is shown by the correlation between $\mathrm{Nb}$ and $\mathrm{Zr}$ on Figure 14, which extends down to very low $\mathrm{Nb}$ abundances. A conservative cut-off below which interelement corrections become significant, is shown. The scatter on this plot is primarily the result of real variations in the $\mathrm{Zr} / \mathrm{Nb}$ ratio.

In this study, rare earth element (REE) plots are normalized against the chondrite abundances of Sun and McDonough (1989), whereas

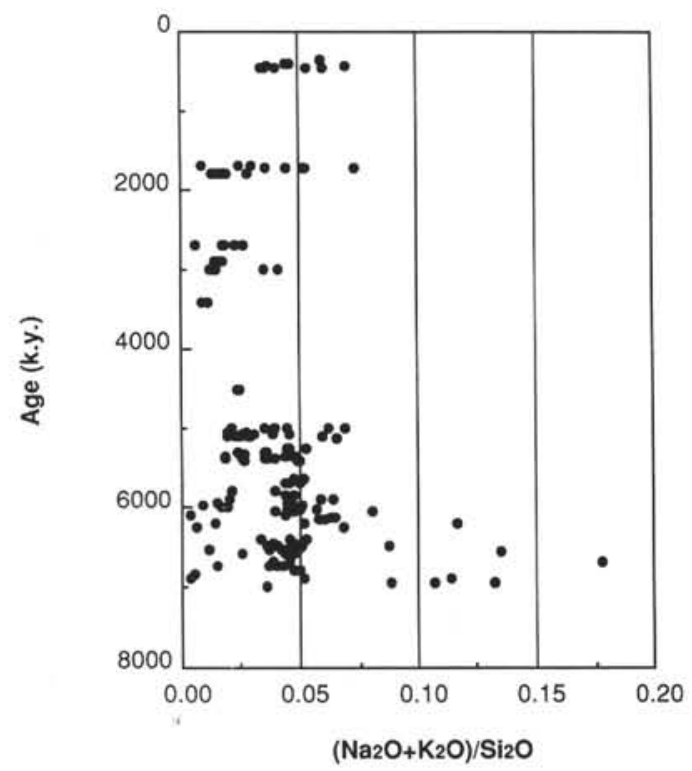

Figure 8. Variation in total alkali content of glass grains with time from the Tonga Platform (Site 840).

trace element spider diagrams, including a REE selection, are normalized against the normal mid-ocean-ridge basalt (N-MORB) concentrations of the same authors. The elements are then plotted along a horizontal axis on which elements mobile in aqueous fluids occupy the left-hand side (as far as Th) and immobile the right (from $\mathrm{Nb}$ ). Compatibility of the elements in mantle phases is arranged to increase to the right among the immobile elements and toward the left in the mobile elements. The REEs are plotted in ascending atomic number order relative to chondrite values.

\section{General Trace Element Characteristics of the Basic Rocks}

Figures 15A, 15B, and 15C are MORB-normalized spider diagrams of the trace element contents of all the basic glasses analyzed from Site 840. Figures 16 and 17 are MORB-normalized spider diagrams for basic glasses from Sites 835,837 , and 839 and from Site 834 , respectively. They show several consistent features, typical of island-arc tholeiites (e.g., Pearce, 1983). The immobile elements from La onward show generally flat patterns most often depleted relative to MORB. A distinct slight relative depletion in $\mathrm{Zr}$ and $\mathrm{Hf}$ compared with adjacent REEs is common. The patterns from Site 840 are 
Table 4 (continued).

\begin{tabular}{|c|c|c|c|c|c|c|c|c|c|c|c|c|c|c|c|}
\hline Hole: & $839 \mathrm{~A}$ & $839 \mathrm{~A}$ & $839 \mathrm{~A}$ & $839 \mathrm{~A}$ & $840 \mathrm{~A}$ & $840 \mathrm{~A}$ & $840 \mathrm{C}$ & $840 B$ & $840 \mathrm{C}$ & $840 B$ & $840 \mathrm{C}$ & $840 \mathrm{~B}$ & $840 B$ & $840 \mathrm{~B}$ & $840 \mathrm{~B}$ \\
\hline Core, section: & $6 \mathrm{H}-5$ & $6 \mathrm{H}-7$ & $10 \mathrm{H}-3$ & $11 X-6$ & $1 \mathrm{H}-1$ & $1 \mathrm{H}-3$ & $1 \mathrm{H}-2$ & $2 X-6$ & $4 \mathrm{H}-4$ & $12 X-5$ & $10 \mathrm{H}-1$ & $33 X-1$ & $42 X-3$ & $55 \times-4$ & $62 X-5$ \\
\hline Interval $(\mathrm{cm})$ : & 136 & 59 & 63 & 82 & 102 & 45 & 29 & 144 & 20 & 50 & 3 & 88 & 3 & 94 & 42 \\
\hline Age (k.y.): & 1450 & 1500 & 1700 & 1750 & 350 & 450 & 1700 & 1700 & 2900 & 5000 & 5300 & 5700 & 6100 & 6700 & 7000 \\
\hline Depth (mbsf): & 49.86 & 52.09 & 84.13 & 98.32 & 1.02 & 3.50 & 39.79 & 18.44 & 71.20 & 110.50 & 171.50 & 309.10 & 388.80 & 515.40 & 584.40 \\
\hline \multicolumn{16}{|c|}{ Major elements (wt\%): } \\
\hline $\mathrm{SiO}_{2}$ & 51.72 & 50.90 & 52.30 & 52.41 & 50.45 & 53.92 & 50.14 & 49.73 & 50.64 & 52.01 & 51.47 & 52.75 & 49.99 & 52.52 & 50.14 \\
\hline $\mathrm{TiO}_{2}^{2}$ & 0.19 & 0.38 & 0.13 & 0.26 & 0.16 & 0.10 & 0.61 & 0.62 & 0.36 & 0.30 & 0.35 & 0.36 & 0.68 & 0.34 & 0.45 \\
\hline $\mathrm{Al}_{2} \mathrm{O}_{3}$ & 0.81 & 1.32 & 0.78 & 1.06 & 0.46 & 0.95 & 3.13 & 4.08 & 1.25 & 0.88 & 2.11 & 1.32 & 2.87 & 1.24 & 2.93 \\
\hline $\mathrm{Fe}_{2} \mathrm{O}_{3}$ & 21.81 & 10.73 & 20.14 & 4.47 & 28.45 & 2.76 & 11.28 & 7.46 & 13.97 & 20.60 & 10.10 & 19.75 & 15.16 & 18.99 & 11.33 \\
\hline $\mathrm{MnO}^{\circ}$ & 0.97 & 0.57 & 0.62 & 0.24 & 1.03 & 0.08 & 0.34 & 0.18 & 0.59 & 1.11 & 0.31 & 0.51 & 0.53 & 0.59 & 0.32 \\
\hline $\mathrm{MgO}$ & 21.49 & 13.45 & 22.27 & 14.97 & 16.53 & 17.59 & 14.34 & 15.88 & 12.04 & 21.80 & 14.41 & 22.29 & 18.30 & 22.51 & 15.94 \\
\hline $\mathrm{CaO}$ & 1.44 & 20.38 & 1.90 & 24.83 & 1.82 & 23.42 & 18.48 & 19.44 & 19.27 & 1.62 & 20.53 & 2.55 & 10.71 & 2.26 & 16.75 \\
\hline $\mathrm{Na}_{2} \mathrm{O}$ & 0.06 & 0.25 & 0.04 & 0.12 & 0.03 & 0.12 & 0.20 & 0.26 & 0.28 & 0.03 & 0.20 & 0.04 & 0.17 & 0.03 & 0.19 \\
\hline $\mathrm{K}_{2} \mathrm{O}$ & 0.01 & 0.00 & 0.01 & 0.00 & 0.01 & 0.00 & 0.01 & 0.01 & 0.00 & 0.00 & 0.00 & 0.00 & 0.02 & 0.00 & 0.01 \\
\hline $\mathrm{P}_{2} \mathrm{O}_{5}$ & 0.03 & 0.36 & 0.04 & 0.41 & 0.00 & 0.38 & 0.29 & 0.35 & 0.00 & 0.06 & 0.31 & 0.07 & 0.18 & 0.00 & 0.25 \\
\hline Total & 98.71 & 98.72 & 98.33 & 99.04 & 99.09 & 99.42 & 99.44 & 98.09 & 98.76 & 98.69 & 100.14 & 100.00 & 99.27 & 98.82 & 98.76 \\
\hline
\end{tabular}

commonly subparallel, reflecting the effects of low-pressure crystal fractionation, which moves the whole pattern parallel to the vertical axis for as long as the elements plotted remain equally incompatible in the crystallizing phases. This is least applicable to $\mathrm{Sr}$ when plagioclase is crystallizing and in consequence the patterns vary least in this element. All patterns have a very prominent relative depletion dip at $\mathrm{Nb}$ and are enriched in $\mathrm{Rb}, \mathrm{Ba}$ and $\mathrm{U}$ relative to MORB.

Figure 18 shows chondrite-normalized REE plots for Site 840 . Figure 18A shows the amount of variation detected at the lowest levels in the section (i.e., upper Miocene), Figure 18B shows the variability at the time of Lau Basin rifting (uppermost late Miocene), and Figure $18 \mathrm{C}$ shows the variability since that time (Pliocene-Pleistocene). The typical form of these diagrams for Site 840 is a straight profile, showing some small variability in the relative enrichment of light compared with heavy REEs. No marked Eu anomalies are present, which would have indicated significant plagioclase fractionation, unless the oxidation state was high enough to convert $\mathrm{Eu}^{2+}$ to $\mathrm{Eu}^{3+}$ to a significant extent. Importantly, none of the Site 840 glasses show the strong relative LREE depletion that typifies N-MORB. The REE patterns are broadly comparable to other low-K tholeiitic arc volcanics and contrast with the stronger enrichment in LREEs found in calc-alkaline basalts. The relatively horizontal trends of the plots would indicate an enrichment in the LREEs relative to MORB, which could indicate enrichment of the source by a slab-derived component or lower degrees of partial melting than for MORB, or both.

Clearly, variations in the level of light REE enrichment are present, from occasionally flat and MORB-like to more commonly slightly enriched in the REEs from La to Sm. It is a matter of observation that the trace element patterns stack together without prominent crossing trends even when of comparable states of low-pressure fractionation. This suggests that the data are not subject to random errors and further, that trends in relative enrichment might apply to all the elements, whether high field strength elements (HFSE), REEs, or "mobile" LILEs. Examination of Figures 16 and 17, and in particular the slopes of the light REE parts of the profiles and the depth of the Nb "dips," shows that, at least at some sites (e.g., Site 837; Fig. 16C), material of both a depleted and a relatively enriched character was being deposited in the same time interval, all of arc-tholeiite character.

A number of questions arise from this. Is the pattern of abundance of incompatible and compatible elements repeated for all elements, whether mobile or immobile, or is there evidence of an independently fluctuating mobile component superimposed on an enrichment and depletion pattern in other elements? Can both effects be identified? The eruption of variably enriched primary melts from a single center could be the result of incomplete mixing of products from deeper in the melt column with melts that arise from higher up in the column, assuming that melting under arcs is governed by the same principles as under a ridge or in a plume (e.g., Spiegelman and McKenzie, 1987). In a mixed-source volcaniclastic sediment, the possibility arises of fundamentally different sources or melting regimes feeding different edifices. To explore these questions, Site 840 , which has the longest age range and which spans the rifting event, was examined in some detail as it is the best candidate for being fed by a small number of closely related arc volcanoes. In having a long age span, as well as a probable single source, it provides the best body of data to test whether the degree of enrichment changes systematically with time.

\section{Trace Element Correlations}

The logarithmic plot of $\mathrm{Zr}$ vs. Nb for Site 840 (Fig. 14) shows that the data are confined within two parallel bounds corresponding to different $\mathrm{Zr} / \mathrm{Nb}$ ratios, varying from approximately 50 at the right hand side to 2000 at the left. Within this range there is a clear relationship between the $\mathrm{Nb} / \mathrm{Zr}$ ratio and the absolute abundance. $\mathrm{The} \mathrm{Nb} / \mathrm{Zr}$ ratio, which measures the degree of high-field-strengthelement enrichment, can be seen to decrease with decreasing abundance of $\mathrm{Nb}$ (Fig. 19). Some of the spread in Figure 19 at any one $\mathrm{Nb} / \mathrm{Zr}$ ratio is because of fractionation. However, $\mathrm{Nb}$ decreases faster than $\mathrm{Zr}$, a fact consistent with a range in the degree of source depletion, or a range in the degree of partial melting, operating on a source with very low effective $\mathrm{Nb}$ concentration. In addition, the implied difference in incompatibility between $\mathrm{Zr}$ and $\mathrm{Nb}$, reflected in the large changes in $\mathrm{Nb}$ concentrations compared to small changes in $\mathrm{Zr}$ content, is very much larger than is the case in melts coming from a more normal MORB source (e.g., McKenzie and Bickle, 1988). This may indicate that the residue assemblage is fundamentally different in melting below arcs, because not only is the source effectively extremely poor in $\mathrm{Nb}$ but the bulk distribution coefficient must also be different. The 30 -fold range in $\mathrm{Nb}$ must imply at least this range in melt-fraction if the bulk distribution coefficient remains constant. This appears unacceptably large for the apparent moderate range in enrichment in other elements.

\section{Reliability of Mobile Element Data}

Figure 20 shows $\mathrm{La} / \mathrm{Sm}$, a measure of relative light REE enrichment, against Rb, for Sites 834-840 together. The positive correlations are good evidence for the same enrichment process governing a mobile element, such as Rb, and the light REEs. As noted earlier it also supports the contention that $\mathrm{Rb}$ is not randomly remobilized on burial.

Figure 21 shows two different ratios, La/Y, effectively a light to heavy $\mathrm{REE}$ ratio, and $\mathrm{Rb} / \mathrm{Sr}$, the ratio of a pair of mobile elements of equivalently different incompatibility, for glass grains at Site 840 . The positive correlation supports the contentions above that a control by melting is affecting all the elements and that mobile elements are retained in the glasses. Figure 22 is a similar plot showing $\mathrm{Nb} / \mathrm{Zr}$ vs. $\mathrm{La} / \mathrm{Sm}$ for the $\mathrm{MgO}>4 \%$ samples (i.e., least evolved) from Site 840 . It shows a positive correlation for relatively immobile element ratios 
Table 5. Representative microprobe analyses of plagioclase feldspars from each of the Lau Basin and Tonga Platform sites (834-840).

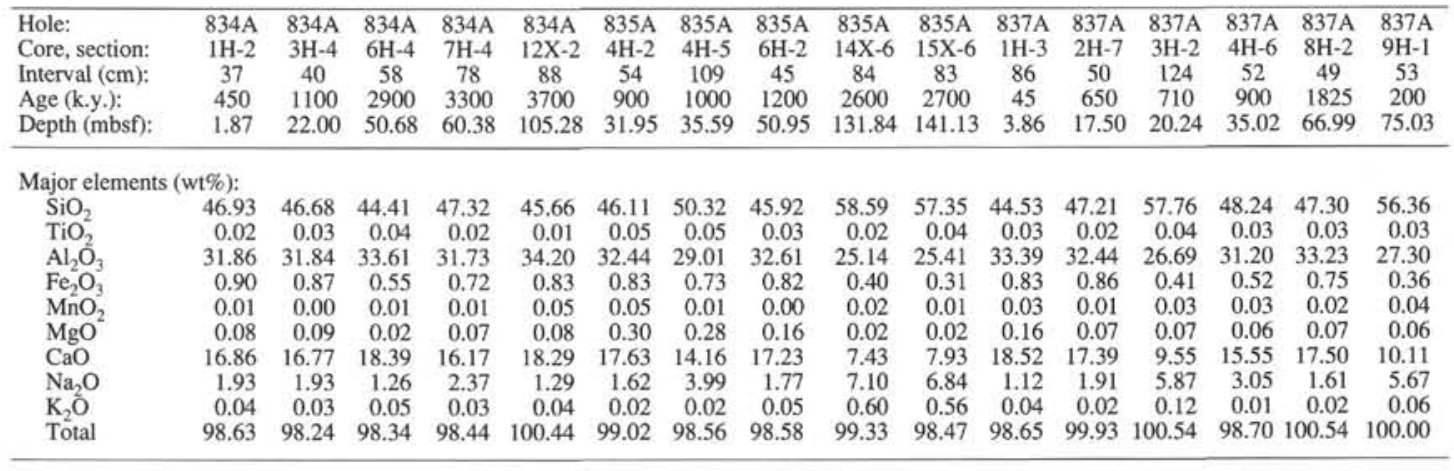

from a data set restricted to compositions for which fractionation is not expected to disturb either ratio (Latin et al., 1990).

It should be noted that these plots are most simply explained by a single enrichment/depletion process related to melting. However, absolute changes in the abundance of some mobile incompatibles could be occurring in the source and might not disturb the correlation, if they happened to occur in the same sense and at the same time as the principal melting process was also causing them to change.

Finally, plotting $\mathrm{Ba} / \mathrm{Sr}$ vs. $\mathrm{Ba}$ for the Site 840 analyses filtered to eliminate the low $\mathrm{MgO}$ samples, shows that again, the levels of the mobile element $\mathrm{Ba}$ are proportional to the degree of enrichment in $\mathrm{Ba}$ relative to the more compatible $\mathrm{Sr}$ (Fig. 23). By itself this simply shows that $\mathrm{Ba} / \mathrm{Sr}$ is dominated by the absolute $\mathrm{Ba}$ level. The extent to which this ratio correlates with $\mathrm{Nb} / \mathrm{Zr}$ and so relates to more than a net influx of $\mathrm{Ba}$ to the source is discussed further below.

This discussion has concentrated on the data from Site 840 because it is the most comprehensive. Our exploration of the more scattered data from the other sites suggests that similar correlations exist between interelement ratios, but the pattern is often less clear.

\section{Variations in Absolute Concentrations and Interelement Ratios with Age and Their Implications}

\section{Site 840-Tonga Platform}

Figure 15 shows trace element spider diagrams for Site 840 arranged in age progression. The predominant feature is one of constancy in the flat part of the immobile incompatible element pattern, with some apparent variation in the light to heavy REE enrichment. However, $\mathrm{Nb}$ clearly becomes less abundant with time in the period up to rifting at approximately $5 \mathrm{Ma}$.

Figure 24 shows that the absolute levels of both $\mathrm{Nb}$ and $\mathrm{Zr}$ decline with time before Lau Basin rifting. Over the 7.0-5.0 Ma interval up to the opening of the Lau Basin, Nb shows a fall from 2.0 to $0.1 \mathrm{ppm}$ or less, whereas $\mathrm{Zr}$ falls from approximately 100 to $20 \mathrm{ppm}$. A decrease in the $\mathrm{Nb} / \mathrm{Zr}$ ratio is apparent over the same period when all the analyses are plotted and is still evident when the data are filtered to exclude possible effects of low-pressure fractionation $(\mathrm{MgO}<4 \%$ are excluded; Fig. 25). The fact that $\mathrm{Nb} / \mathrm{Zr}$ falls over a time interval when $\mathrm{Zr}$ is falling, indicates that it is the more rapid falling levels of $\mathrm{Nb}$ that are responsible for controlling $\mathrm{Nb} / \mathrm{Zr}$. It should be noted that these changes are not the result of some systematic change in the degree of low-pressure fractionation and consequent sampling bias with time. The systematic behavior of $\mathrm{Nb}$ and $\mathrm{Zr}$, and of $\mathrm{Nb} / \mathrm{Zr}$ with age, implies an evolving parental magma composition with time. Latin et al. (1990) argue that $\mathrm{Zr} / \mathrm{Nb}$ variations, which are unaffected by moderate degrees of fractionation or alteration, are indicators of the extent of melting of the source or its level of prior depletion. The data presented here are compatible with a progressive increase in one or both of these parameters before basin opening.
The problem of accounting for, by a single process, both the extremely depleted levels of $\mathrm{Nb}$ in the melt and the further decrease in $\mathrm{Nb}$ abundance from these levels, in parallel with $\mathrm{Zr}$, but some 2 orders of magnitude more effectively, has already been alluded to. This process would now appear to be one that changes systematically with time, as $\mathrm{Nb}$ behaves as an incompatible element. If it was merely severely depleted in a mantle wedge otherwise identical to a MORB source, then melting would deplete it further. The factor of 30 increase is implied in the melt-fraction when the change in $\mathrm{Zr} / \mathrm{Nb}$ values is considered in the light of the partition coefficients used by McKenzie and Bickle (1988). Such a large increase is geologically unrealistic over this period. An effective change in the bulk distribution coefficient is implied, with $\mathrm{Nb}$ effectively becoming more and more compatible in the residue with melting. A phase that takes up $\mathrm{Nb}$ and increases in abundance with melting is a possibility and rutile is a potential candidate.

The trend to increasing depletion in HFSEs up to the point of rifting seems reasonably clear, but more data from mafic compositions would be welcome. At Site 840 , a hiatus occurs in the volcaniclastic input between 5.0 and $3.0 \mathrm{Ma}$. After the initiation of the Tofua Arc at 3.0 Ma, the trend in composition is less clear because of the scarcity of data, but it is not inconsistent with a return to more enriched conditions. It should be noted that the age attributed to a given sample is that derived from the microfauna, either from the volcaniclastic sand or from the interbedded pelagic intervals. The possibility that rifting of the old forearc exposed older volcaniclastics is a real one. The two samples that lie off the depletion trend for $\mathrm{Nb} / \mathrm{Zr}$ are those for the time of inferred rifting itself, and it is tempting to conclude that they are reworked.

Plotted beside $\mathrm{Nb} / \mathrm{Zr}$ are the results for the filtered $\mathrm{MgO}>4 \%$ set from Site 840 , showing $\mathrm{La} / \mathrm{Sm}$ and $\mathrm{Ba} / \mathrm{Sr}$ vs. age as before (Fig. 25). The same pattern is seen of depletion followed by a weak trend to enrichment. In each plot, the two analyses from the time of rifting lie off the depletion trend, in a sense consistent with their being reworked older material. The pattern in the postrift samples is also the same in each plot, despite the values for the ratios being determined independently of each other. What is also clear is that the extent to which the ratios return to, or even exceed, the values they had in the oldest samples, in the youngest samples, varies between the plots. It is least for $\mathrm{Nb} / \mathrm{Zr}$, moderate for $\mathrm{La} / \mathrm{Sm}$, and greatest for $\mathrm{Ba} / \mathrm{Sr}$.

In general terms, the fact that the ratios correlate with each other when the data are amalgamated leads one to expect that an age trend in one will be reflected in another. The additional factor apparent in the age plots, that $\mathrm{Ba}$ is more enriched relative to $\mathrm{Sr}$ in the youngest samples than it was in the older samples and that this is not apparent in $\mathrm{Nb} / \mathrm{Zr}$, would appear in an ideal way as a hairpin loop on a $\mathrm{Ba} / \mathrm{Sr}$ vs. $\mathrm{Nb} / \mathrm{Zr}$ plot. More probably, it would be manifest as a broader positive correlation than for two immobile element pairs.

The best test of changes in source composition, or of changes in the pattern of enrichment of the melts that are independent of the extent 
Table 5 (continued),

\begin{tabular}{|c|c|c|c|c|c|c|c|c|c|c|c|c|c|c|c|c|}
\hline Hole: & $838 \mathrm{~A}$ & $838 \mathrm{~A}$ & $838 \mathrm{~A}$ & $839 \mathrm{~A}$ & $839 \mathrm{~A}$ & $839 \mathrm{~A}$ & $839 \mathrm{~A}$ & $839 \mathrm{~A}$ & $839 \mathrm{~A}$ & $840 \mathrm{~B}$ & $840 \mathrm{C}$ & $840 \mathrm{C}$ & $840 \mathrm{C}$ & $840 \mathrm{~B}$ & $840 \mathrm{~B}$ & $840 B$ \\
\hline Core, section: & $7 \mathrm{H}-2$ & $7 \mathrm{H}-2$ & $11 X-4$ & $1 \mathrm{H}-3$ & $4 \mathrm{H}-3$ & $6 \mathrm{H}-1$ & $7 \mathrm{H}-2$ & $12 \mathrm{X}-1$ & $18 \mathrm{X}-\mathrm{CC}$ & $1 \mathrm{X}-\mathrm{CC}$ & $1 \mathrm{H}-2$ & $4 \mathrm{H}-6$ & $10 \mathrm{H}-1$ & $38 X-1$ & $51 X-5$ & $62 X-5$ \\
\hline Interval $(\mathrm{cm})$ : & 69 & 69 & 30 & 44 & 126 & 117 & 101 & 1 & 6 & 33 & 29 & 34 & 3 & 17 & 10 & 42 \\
\hline Age (k.y.): & 1700 & 1700 & 2000 & 300 & 1000 & 1500 & 1650 & 1700 & 1750 & 400 & 425 & 3000 & 5300 & 5950 & 6500 & 7000 \\
\hline Depth (mbsf): & 53.39 & 53.39 & 94.00 & 3.44 & 29.26 & 43.67 & 54.51 & 99.51 & 167.26 & 1.68 & 2.25 & 74.34 & 171.50 & 347.30 & 477.80 & 584.40 \\
\hline \multicolumn{17}{|c|}{ Major elements (wt\%): } \\
\hline $\mathrm{SiO}_{2}$ & 54.23 & 55.03 & 54.95 & 48.93 & 49.23 & 48.15 & 49.53 & 46.03 & 53.07 & 53.03 & 48.45 & 45.59 & 46.52 & 46.68 & 56.01 & 55.21 \\
\hline $\mathrm{TiO}_{2}^{2}$ & 0.04 & 0.06 & 0.03 & 0.02 & 0.03 & 0.01 & 0.04 & 0.04 & 0.05 & 0.05 & 0.05 & 0.03 & 0.04 & 0.04 & 0.05 & 0.06 \\
\hline $\mathrm{Al}_{2} \mathrm{O}_{3}$ & 27.32 & 27.23 & 27.39 & 30.67 & 31.19 & 31.51 & 30.80 & 32.96 & 28.81 & 28.63 & 32.26 & 33.70 & 32.93 & 33.32 & 27.46 & 27.13 \\
\hline $\mathrm{Fe}_{2} \mathrm{O}_{3}$ & 0.54 & 0.50 & 0.56 & 0.70 & 0.52 & 0.69 & 0.76 & 0.65 & 0.55 & 1.40 & 0.77 & 0.86 & 1.25 & 0.69 & 0.55 & 0.63 \\
\hline $\mathrm{MnO}_{2}$ & 0.02 & 0.01 & 0.02 & 0.00 & 0.02 & 0.00 & 0.02 & 0.00 & 0.00 & 0.03 & 0.02 & 0.02 & 0.03 & 0.01 & 0.00 & 0.01 \\
\hline $\mathrm{MgO}^{-}$ & 0.06 & 0.07 & 0.06 & 0.07 & 0.04 & 0.08 & 0.07 & 0.03 & 0.04 & 0.22 & 0.06 & 0.18 & 0.11 & 0.19 & 0.08 & 0.07 \\
\hline $\mathrm{CaO}$ & 10.97 & 10.41 & 10.85 & 15.14 & 15.08 & 15.58 & 14.78 & 17.23 & 12.10 & 13.52 & 16.25 & 18.61 & 17.70 & 17.89 & 10.67 & 10.70 \\
\hline $\mathrm{Na}_{2} \mathrm{O}$ & 5.37 & 5.90 & 5.54 & 3.08 & 3.24 & 2.72 & 3.27 & 1.91 & 4.91 & 3.93 & 2.38 & 1.06 & 1.54 & 1.62 & 5.83 & 5.54 \\
\hline $\mathrm{K}_{2} \mathrm{O}$ & 0.09 & 0.08 & 0.05 & 0.02 & 0.04 & 0.03 & 0.03 & 0.01 & 0.05 & 0.11 & 0.02 & 0.02 & 0.04 & 0.03 & 0.09 & 0.08 \\
\hline Total & 98.64 & 99.29 & 99.43 & 98.63 & 99.38 & 98.78 & 99.29 & 98.86 & 99.57 & 100.92 & 100.26 & 100.06 & 100.14 & 100.47 & 100.73 & 99.43 \\
\hline
\end{tabular}

of melting, is to examine the changes with time in the ratio of two elements that are of similar incompatibility but of different mobility.

Figure 26 , which shows $\mathrm{Ba} / \mathrm{Nb}$ vs. La/Nb, clearly demonstrates that the modern Tofua Arc data (from Ewart et al., this volume) and the majority of the Lau Basin data lie on curves of different $\mathrm{Ba} / \mathrm{La}$ ratio. However, within each group of analyses, this ratio is constant. The modern arc points lie close to a curve of $\mathrm{Ba} / \mathrm{La}=50$. The dispersion of the data points along this curve must be caused by variations in $\mathrm{Nb}$ and thus in $\mathrm{La} / \mathrm{Nb}$ and $\mathrm{Ba} / \mathrm{Nb}$. It may also be a result of variations in $\mathrm{Ba}$ and $\mathrm{La}$ coupled to those in $\mathrm{Nb}$, consistent with varying enrichment as in a melting process, or it may be a result of fluctuations in absolute $\mathrm{Ba}$ and $\mathrm{La}$ together, against a constant background value of $\mathrm{Nb}$ for parental tholeiites. The Lau Basin/Tonga Platform data lies close to a curve of $\mathrm{Ba} / \mathrm{La}=20$, with the most recent samples rising upward from this and overlapping the Tofua Arc field. The dispersion of the Lau Basin/Tonga Platform data is at least in part caused by the coupled behavior of HFSEs and REEs, with variations in $\mathrm{Ba} / \mathrm{Nb}$ and $\mathrm{La} / \mathrm{Nb}$ being matched by variations in $\mathrm{Zr} / \mathrm{Nb}$. The behavior of $\mathrm{Ba}$ at Site 840 before rifting indicates that its concentration went down and, with it, levels of $\mathrm{Ba} / \mathrm{Sr}$. What has happened since is that $\mathrm{Ba} / \mathrm{Sr}$ "recovered" more strongly than $\mathrm{Nb} / \mathrm{Zr}$ and overtook its original $7.0 \mathrm{Ma}$ values. The $\mathrm{Ba} / \mathrm{La}$ data suggest that this must be the result of preferential enrichment of $\mathrm{Ba}$ in the source relative to $\mathrm{La}$. In addition, the behavior of the $\mathrm{La} / \mathrm{Sm}$ ratio at Site 840 suggests that this active enrichment process extends to the lighter rare earth elements being enriched relative to the heavier rare earths. A flux of mobile elements from the slab is the obvious source of this extra component of enrichment.

Figure 27 is a plot of $\mathrm{Ba} / \mathrm{Zr}$ vs. age for all the Lau Basin and Tonga Platform data. Because $\mathrm{Zr}$ is somewhat more compatible than $\mathrm{Ba}$, this plot is recording the effect of melt-related enrichment and depletion as well as any absolute changes in a mobile element. Nevertheless, it illustrates very strikingly that the samples from Site 840 show a very marked shift to higher values after rifting. Before rifting (7.0-5.0 Ma), the data from Site 840 show a nearly constant value. Values for $\mathrm{Ba}$ declined during this period, as did values for $\mathrm{Zr}$, and the ratio remained nearly constant. The $\mathrm{Ba} / \mathrm{La}$ values for the early samples at Site 840 are also closely similar (Fig. 28). The simplest explanation is that the slab flux was constant or declined during this period and that the enrichment trends were dominated by the increase in the degree of melting with rifting or the equivalent overall depletion in the source. After rifting, the broad range of $\mathrm{Ba} / \mathrm{Zr}$ ratios apparent at Sites $834-840$ taken together could be caused by the variable influence of the slab flux or variations in the extent of melting, which would cause the $\mathrm{Ba} / \mathrm{Zr}$ ratio to change, or both. As the majority of the sites providing postrifting data are from within the basin on new or attenuated lithosphere, it seems unlikely that the degree of melting is the primary factor. However, it is not clear whether it is the pattern of contemporaneous slab-derived enrichment or the distribution of past enriched and depleted regions contributing to basin magmas, that is responsible.

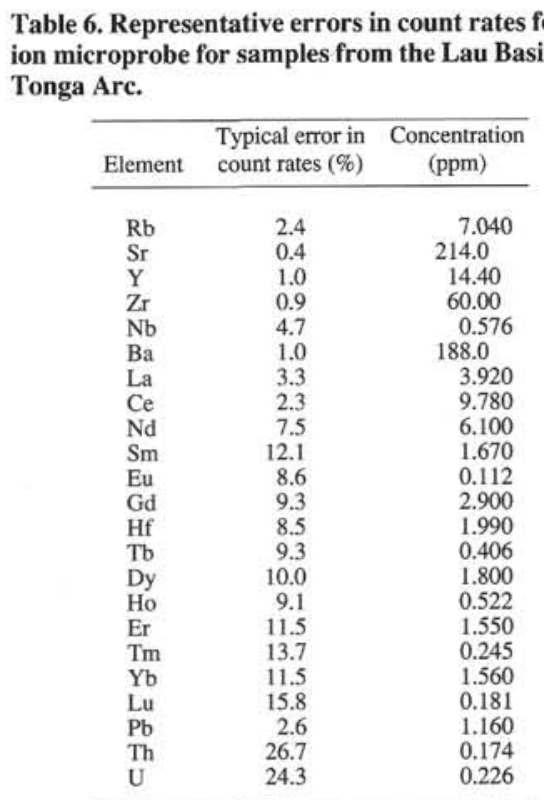

Notes: Analyses from Sample 135-840A-1H-3, $45 \mathrm{~cm}$; Hf values from Sample 135-840B-35X-3, $22 \mathrm{~cm}$.

The value of $\mathrm{Ba} / \mathrm{La}$ characterizing the present Tofua Arc is higher than that in the oldest samples from the Tonga Platform site analyzed. Whether it has returned to a value corresponding to that in an earlier phase (pre-7.0 Ma) of the Lau Ridge (Miocene Tonga Arc) is not known for certain, although provisional analyses from 9.0 Ma glass grains at Site 841 (Fig. 1; Tonga forearc) support this theory by showing Ba/La values around $60-65$ before arc rifting (unpublished data).

\section{Trace Element Variations with Age at Sites 834-839}

Trace element spider diagrams from Sites 835,837 , and 839 (Fig. 16) show variations explicable in terms of the fractional crystallization of a uniform parent magma (e.g., Site 837, late Pliocene), which displaces the patterns parallel to one another. Apparent independent variations in LREE to HREE enrichments are recognized, superimposed on a background of HFSE depletion and very low $\mathrm{Nb}$ values (e.g., compare Samples 135-837A-3H-1, $86 \mathrm{~cm}$, and $-6 \mathrm{H}-4$, $13 \mathrm{~cm}$ ). The three Site 837 samples of late Pliocene age, for example, all happen to show moderate LREE enrichment, although LREEdepleted samples may well be present but not yet sampled. Figure 29 shows the range of $\mathrm{Nb} / \mathrm{Zr}$ values for Sites 834-839 in an attempt to show the degree of HFSE enrichment during the period of basin rifting. A range of $\mathrm{Nb} / \mathrm{Zr}$ values can often be seen over a short time 


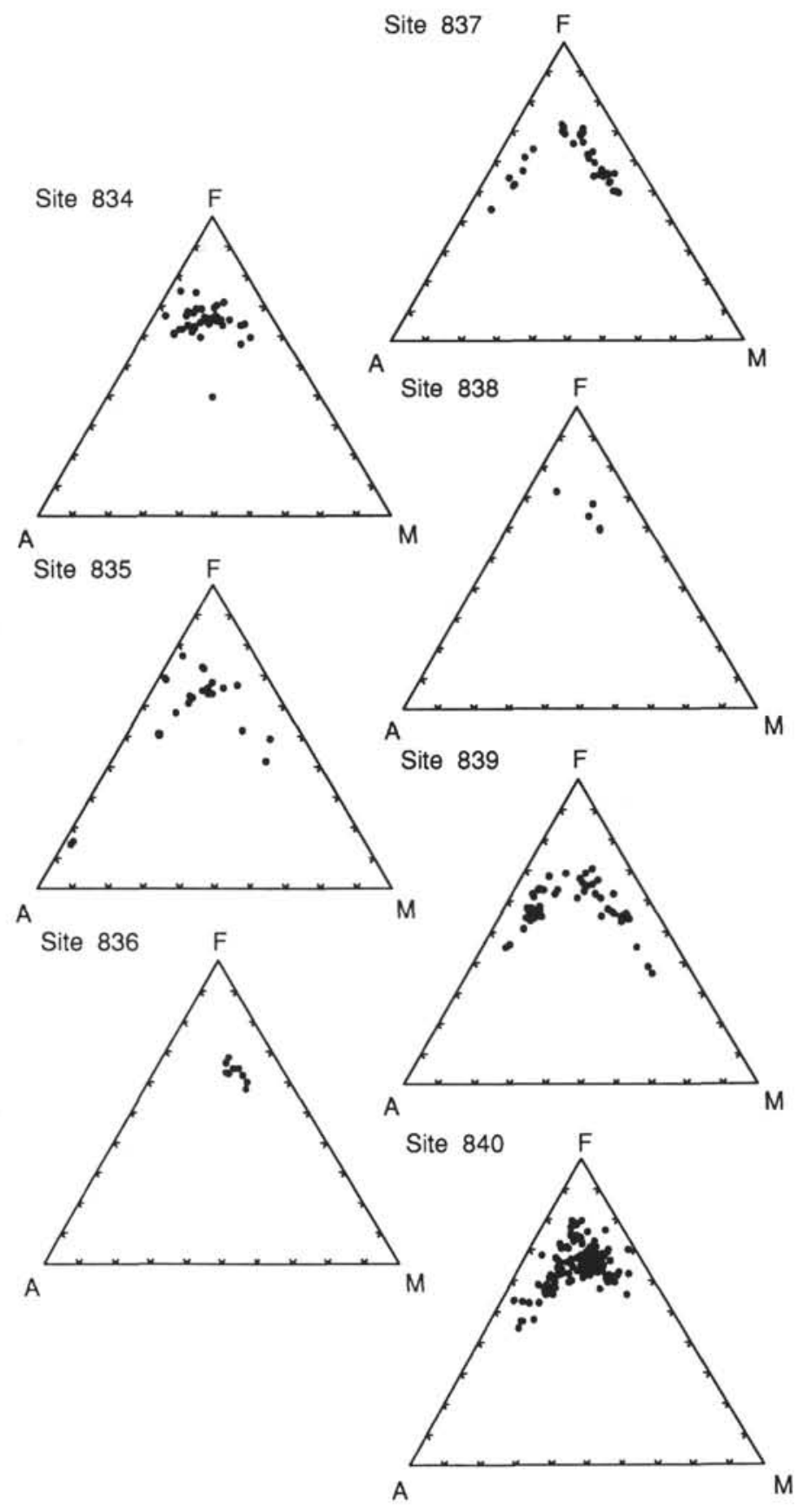

Figure 9. Triangular alkali/iron/magnesium (AFM) diagram showing the vast majority of glass grains from Sites 834 to 840 falling within the tholeiite field, as defined by Irvine and Baragar (1971).

period, which suggests that the simple trend in volcanic chemistry to more depleted melts seen before 5.0 Ma at Site 840 is broken down following arc rifting. Given that the basin sediments represent the activity of seamount volcanism, depletion of the sub-backarc basin mantle would seem to be heterogeneous following arc rifting, with both depleted and enriched regions active over short time periods.

An exception to this general pattern can be found at Site 834, however (Fig. 1). Because this site lies on the flanks of the remnant arc (Lau Ridge) and is the oldest site in the basin, the sediments recovered might also be expected to record the volcanic history of the Lau Ridge from the earliest basin rifting to the present. The oldest material analyzed from Site 834 shows two types of relatively flat trends (Fig. 17). The first type, with a very pronounced $\mathrm{Nb}$ depletion (e.g.,
A

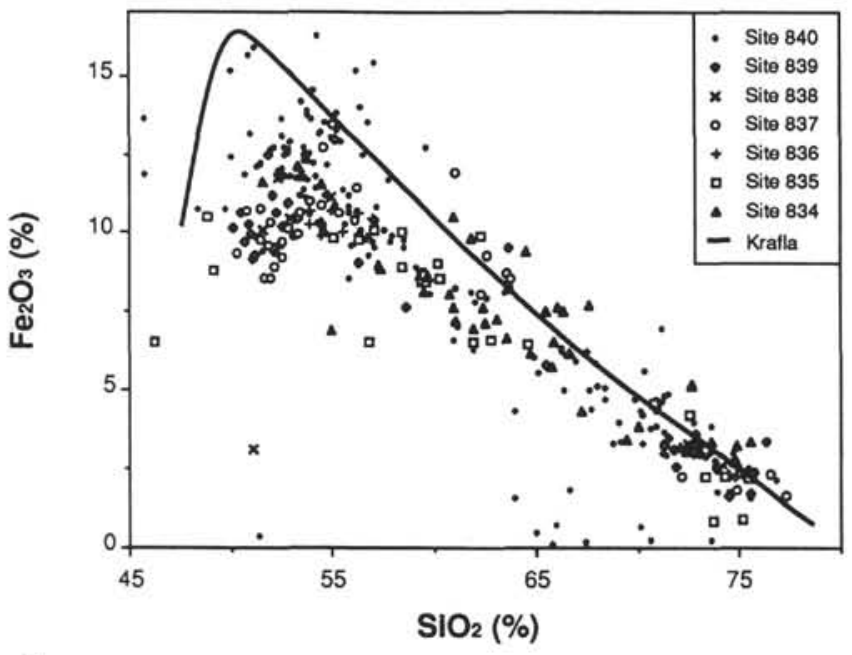

B

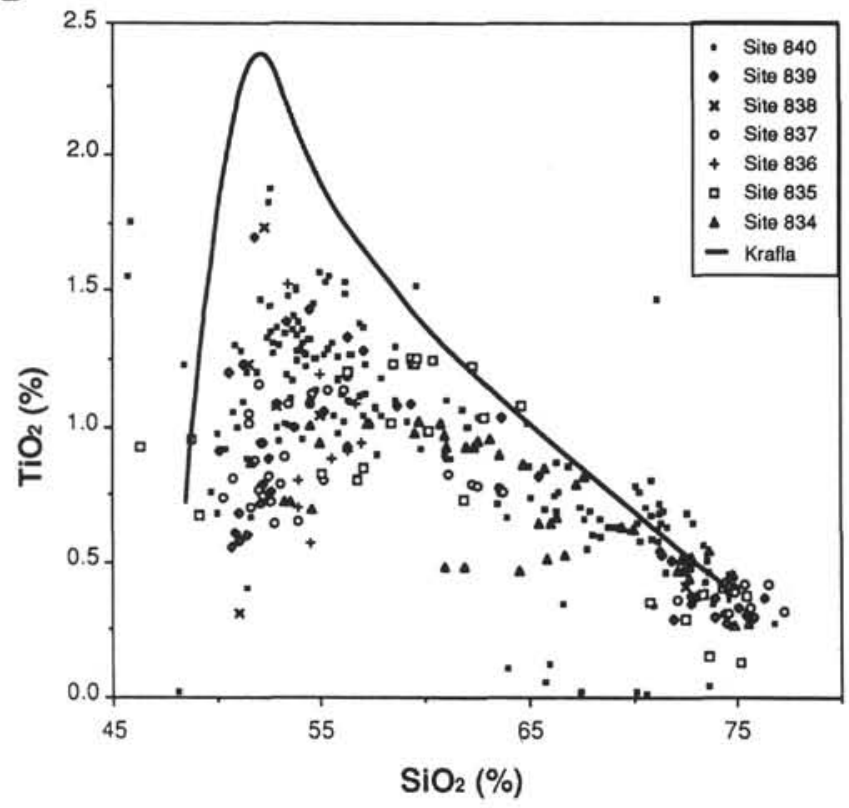

Figure 10. Diagram showing the relationship of silica to $\mathrm{Fe}_{2} \mathrm{O}_{3}(\mathrm{~A})$ and $\mathrm{TiO}_{2}(\mathbf{B})$ for glass grains from Sites 834 to 840 . Tholeiitic fractionation trends from Krafla, Iceland (Fitton and Nicholson, unpubl. data) are provided for comparison.

Sample $135-834 \mathrm{~A}-10 \mathrm{H}-\mathrm{CC}, 6 \mathrm{~cm}$ ), is identical to those found at about $5.0 \mathrm{Ma}$ at Site 840 (Fig. 15B) and was probably derived from the same arc source. A second type, deposited at effectively the same time, shows much less $\mathrm{Nb}$ depletion and a mild enrichment in $\mathrm{La}, \mathrm{Ce}$, and Nd relative to MORB (e.g., Sample 135-834A-10H-3, $102 \mathrm{~cm}$ ). This represents an end, therefore, to the ordered trend to increasing depletion seen in the arc before basin rifting. Later Pleistocene samples from Site 834 (Fig. 17B) show anomalous patterns with very low $\mathrm{Nb}$ but conspicuous depletion of intermediate incompatible elements, reminiscent of boninite patterns (e.g., Simonian, 1975). Evidence is also present for departures from the normal $\mathrm{Zr} / \mathrm{Hf}$ ratios. Figure 30 shows the REE compositions for basaltic glasses at Site 834 normalized against chondrite (Sun and McDonough, 1989). Here again the basic pattern of a mixture of both LREE-enriched and -depleted patterns is recognized in glasses recovered from deeper in the section, as it was at Site 840 . However, the younger samples give analyses with unusual REE patterns. In the case of Sample 135-834A-2H-1, $37 \mathrm{~cm}$, the LREEs and HREEs show similar degrees of concentration 


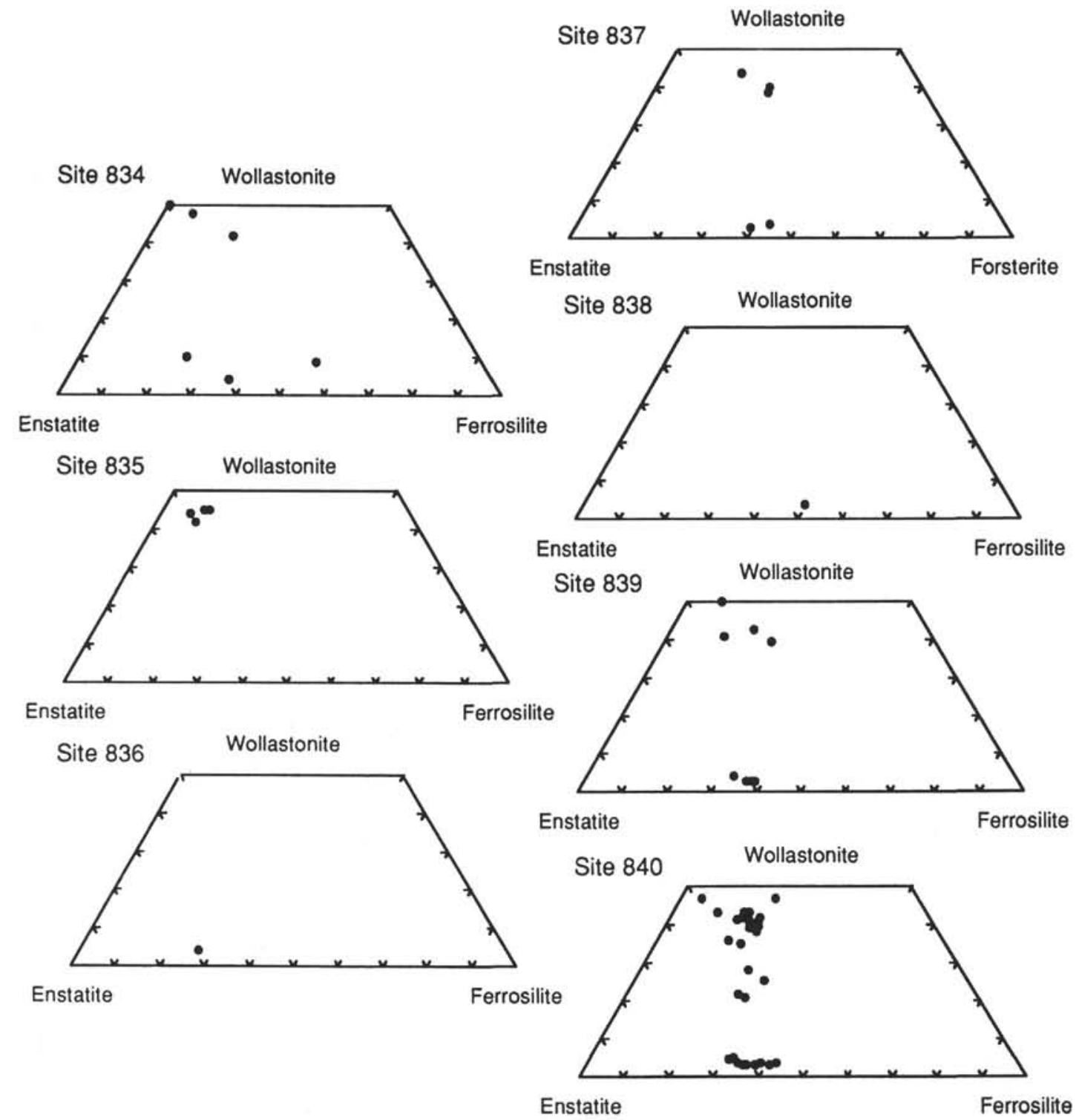

Figure 11. Compositional plot of pyroxene analyses from Sites 834 to 840 on an enstatite/diopside/hedenbergite/ferrosilite trapezoid showing the bimodal split into a Ca-poor and Ca-rich trend, typical of tholeitic magma series.

relative to chondrite. In contrast, Eu and $\mathrm{Tb}$ are depleted relative to the other elements, whereas Dy and Ho are markedly enriched. Sample $135-834 \mathrm{~A}-3 \mathrm{H}-1,72 \mathrm{~cm}$, differs in having a more regular depletion of the intermediate REEs compared with the LREEs and HREEs. The U-shaped pattern seen in some of the spider diagrams (Fig. 17B) is not recognized in the REE diagrams; these glasses, therefore, are not considered to be boninitic. The significance of this behavior is not clear, but it does illustrate the separate source of sediment at Site 834 compared with the other backarc basin sites.

\section{INTERPRETATION AND DISCUSSION}

The chemical compositions of volcanic glass, pyroxene and plagioclase grains recovered from the Lau Basin have important implications for the source and distribution patterns of sediment, as well as the volcanic history of the arc and adjacent basins. The almost total dominance of tholeiitic glass and associated mineral phases clearly indicates that volumetrically, calc-alkaline lavas have been insignificant in arc volcanism before and throughout basin opening. In the case of the major element chemistry of the volcanics, one can see that a changeover occurs from the eruption of a complete range of silica contents both before and immediately after basin rifting to a silica bimodal volcanism later in the rifting. This changeover is accompanied by the demise of high-K volcanism, so that Pliocene-Pleistocene volcanism is characterized solely by low-K tholeiite lavas. The significance of the trend in silica contents is not clear. In some way, parental magma water contents, enrichment characteristics, and viscosities may be interlinked, resulting in progressive changes in the patterns of fractionation and eruption that correlate with more subtle but fundamental changes in the trace-element composition.

In summary, the period up to rifting is characterized at Site 840 by increasing depletion in all incompatible elements, consistent with an increased extent of melting, or source depletion. In detail, this process must differ in important but still poorly defined respects from the behavior of MORB-source mantle as upwelling proceeds in an extending rift. The relative depletion in $\mathrm{Nb}$ characteristic of arc magmas increases during this process, whereas the enrichment in Ba relative to $\mathrm{Sr}$, also typical of arc magmas, decreases. After rifting enrichment 


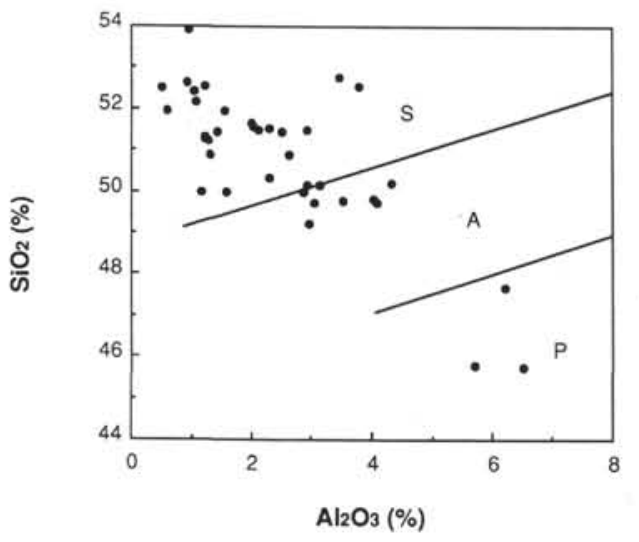

Figure $12 . \mathrm{SiO}_{2}-\mathrm{Al}_{2} \mathrm{O}_{3}$ covariation diagram for detrital clinopyroxene analyses from Sites 834 to 840 . Boundaries taken from Le Bas (1962); $\mathrm{S}=$ subalkaline, $\mathrm{A}=$ alkaline, and $\mathrm{P}=$ peralkaline.

trends reverse but an additional influence is clearly apparent in that the ratio of elements of similar degrees of incompatibility then changes in a way related to their mobility in aqueous fluids. This is shown by the marked increase in $\mathrm{Ba} / \mathrm{La}$ ratios from relatively constant, low values before rifting. This is most easily explained as being caused by an increasing contribution from a slab flux superimposed on a reduction in the extent of melting, which causes the immobile incompatible ratios to return to their previous values.

Within the basin, the wide range in $\mathrm{Ba} / \mathrm{Zr}$ and in incompatible enrichment generally among the different sites is best attributed to the combined effects of variable prior enrichment and depletion in the melt source regions and a variable slab-flux contribution.

\section{Interpretation of the Lau Basin Sediments}

Based on the sedimentary evidence there is good reason to believe that seamount volcanoes within the Lau Basin were active between the uppermost Miocene and middle Pleistocene. The seamounts would have provided, by the process of submarine eruption, the copious amounts of volcanic debris seen in the nearby sub-basins. Any airfall ashes deposited from the adjacent arc during this time would have been reworked by the proximal turbidity currents and were not preserved. However, the volume of sedimentation at Site 840 is in any case much reduced after Lau Basin rifting (approximately 5.0 Ma), when rates of backarc sedimentation were at a peak. This fact rules out the Tofua Arc as a source for this sediment. Even after its foundation, the contribution of the Tofua Arc to the sediment budget of the basin is rather limited. The later sedimentary history at each site shows a marked change in facies towards finer grained clastic sediments and a dominant pelagic component. This shift reflects the migration of the active volcanic zone within the basin towards the east and the end of sedimentation from these sources. However, it is only after this shift to more pelagic sedimentation that it is possible to identify distal airfall ash layers derived from a new subaerial Tofua Arc. The change from regional extension to extension along the Eastern Lau Spreading Center does not strongly alter the sedimentation at preexisting sites, but is manifest in the earliest sediments deposited at newly created sub-basins.

Supporting evidence from the major and trace element chemistries of glasses extracted from Lau Basin volcaniclastic sediments also points toward the majority of them being derived principally from relatively local intrabasinal seamount volcanoes during the rifting of each sub-basin (Fig. 31). Calc-alkaline lavas erupted on the Lau Ridge as part of the middle Miocene Lau Volcanic Group, as well as their reworked equivalents, the shoshonitic and high-K glasses extracted from the early upper Miocene of Site 840, do not seem to have made a significant contribution to the sediments deposited during PliocenePleistocene times. This is the first strong indication that the cycling
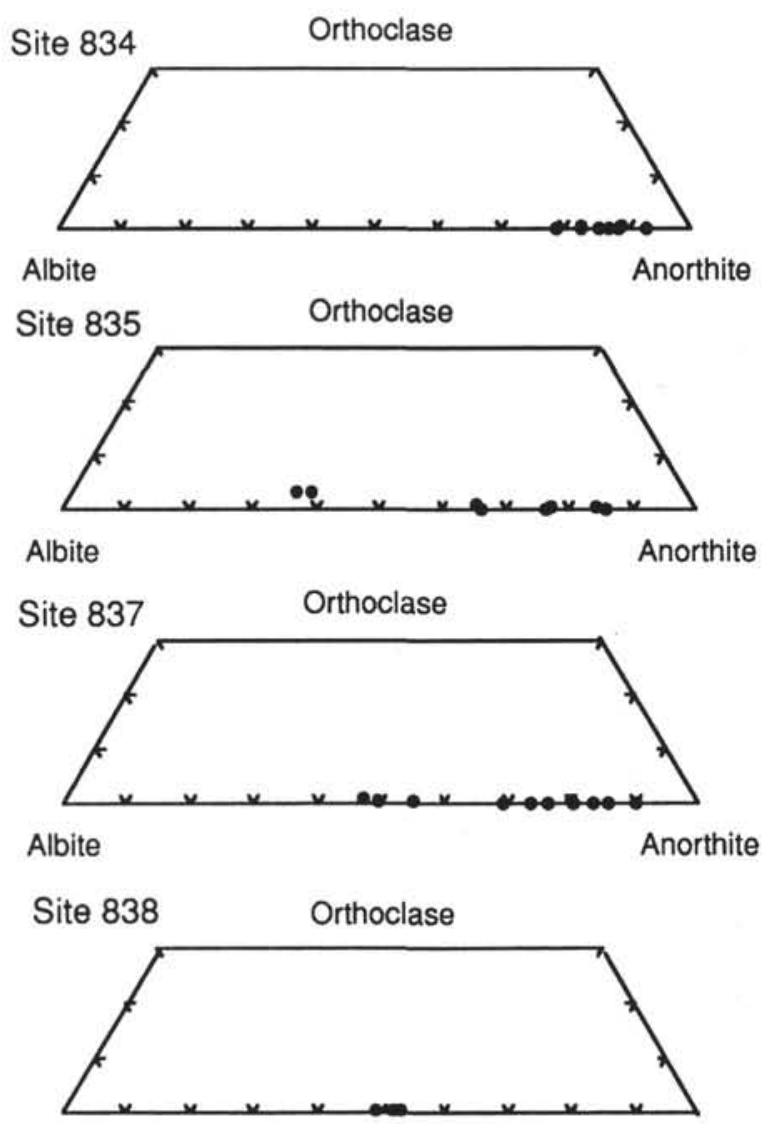

Albite

Anorthite
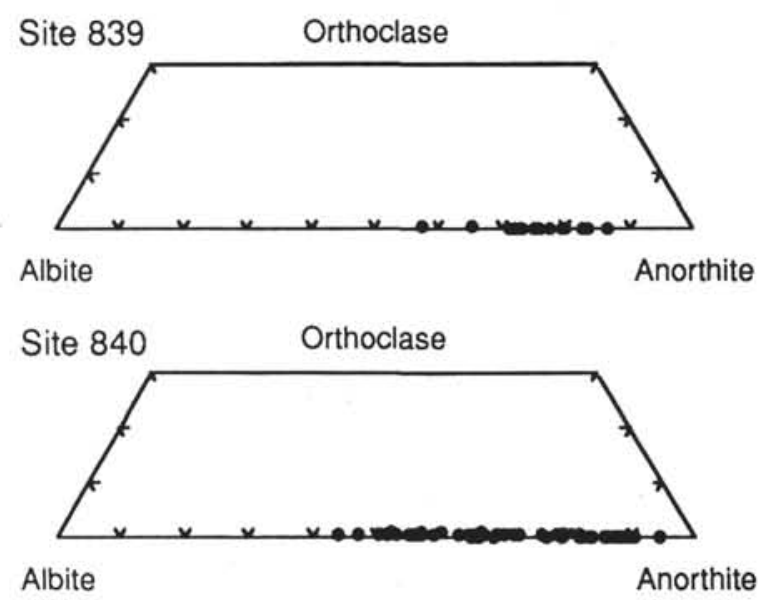

Figure 13. Plagioclase compositions from Sites 834, 835, and 837-840, showing the absence of alkali feldspars and the range in plagioclase compositions.

time for sediments between their initial eruption from a volcano to their redeposition into a given sub-basin is relatively short. The pattern of total silica content of the glasses supports this general scenario, as the sites from the eastern Lau Basin (i.e., Sites 837-839) generally show a bimodal silica distribution, as does Site 840, after basin rifting (i.e., after around 5.0 Ma). The Miocene volcanic history recorded at Site 840, with its spectrum of silica values, does not seem to contribute to the sediments of the central Lau Basin to any large extent, although seamount volcanism of a similar character may be an appropriate source for the sediments deposited at Sites 834 and 835. Some localized reworking of the earlier arc edifice during the 


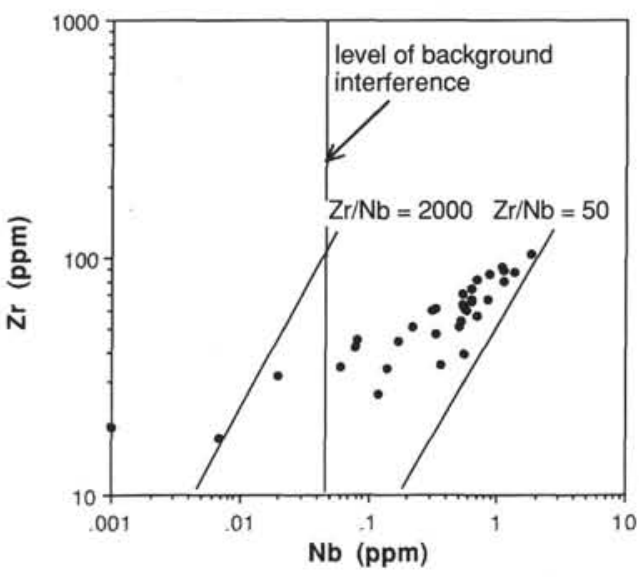

Figure 14. Variations in the concentrations of $\mathrm{Zr}$ with $\mathrm{Nb}$ contents from basaltic andesitic glass grains at Site 840. Note the excellent correlation, and inferred high analytical precision, to very low concentrations of $\mathrm{Nb}(<0.5 \mathrm{ppm})$.

initial opening of the backarc basin is suggested by the presence of tschermakitic clinopyroxenes at the base of the section at Site 835, as well as by the presence of rare calc-alkaline glass grains at the base of the section at Sites 834. It could also explain the minor deviations in the Site 840 data from smooth trends, at the time of rifting. Volumetrically, however, the contribution of these sources seems to be small.

Figure 26 shows the fit, or lack of it, between basement chemistry and the associated sediments recovered above it, in terms of $\mathrm{Ba} / \mathrm{Nb}$ and $\mathrm{La} / \mathrm{Nb}$ ratios. This diagram demonstrates that the cover and basement compositions together span a broad range. As noted earlier, $\mathrm{Ba} / \mathrm{La}$ increases upsection from $76.0 \mathrm{mbsf}$ (3.0 Ma; Fig. 28) and is a reasonable measure of a slab-derived flux. Depletion in $\mathrm{Nb}$, at any given level of $\mathrm{La}$ or Ba causes the plotted composition to migrate up and to the right. MORB lies close to the lower left corner of the diagram. In general, if the sediments at one site are derived short distances from an active volcanic edifice on the flanks of a rifting sub-basin it might be expected that the chemistry of the ashes and basement basalts would be similar. If there is a significant time delay between generation of the sub-basin basement and eruption of the volcaniclastic debris then there is no reason to expect a correspondence in the chemistries of the sediments and the basement. However, the eastward migration of extension with time, mirrored by a similar progression in the locus of active volcanism suggests that such a time delay is short. The basement basalts at Sites 835 and 836 both show strong similarities to the basaltic glass grains in their cover sediments. However, the sediments and basalts are radically different between each site in terms of both their major (Fig. 5) and trace element chemistry. The chemical data thus supports the idea of locally derived sediments sourced from volcanoes of similar geochemistry to the basin floor basalts. Site 834 is very different, having a much more arc-like cover than its basement. This phenomena is probably attributable to its proximity to the Lau Ridge. The cover for Site 839 cover has comparable $\mathrm{Ba} / \mathrm{La}$ to its basement; however, in the ratio of $\mathrm{Nb}$ to these elements, the cover is more variable. Nevertheless, the volcano that produced the ashes at Site 839 seems to have a similar mantle source to the basement. Interestingly, the strongly arc-like character of the ashes at Site 839 makes them indistinguishable from the true arc-derived ashes of Site 840 . This does not imply the same source for the material, but merely that the intrabasinal volcanism in the early Lau Basin is geochemically identical to a normal island arc. Ash from Site 837 also has strongly arc-like characteristics. As discussed above, this probably reflects derivation of this material from the same, or similar, volcano as the ashes at Site 839. In this way the glass chemistry can be reconciled with the differing basement chemistry at Site 837. The deviation of the suite of glasses at Site 840 from the modern Tofua Arc has already been remarked upon.
A

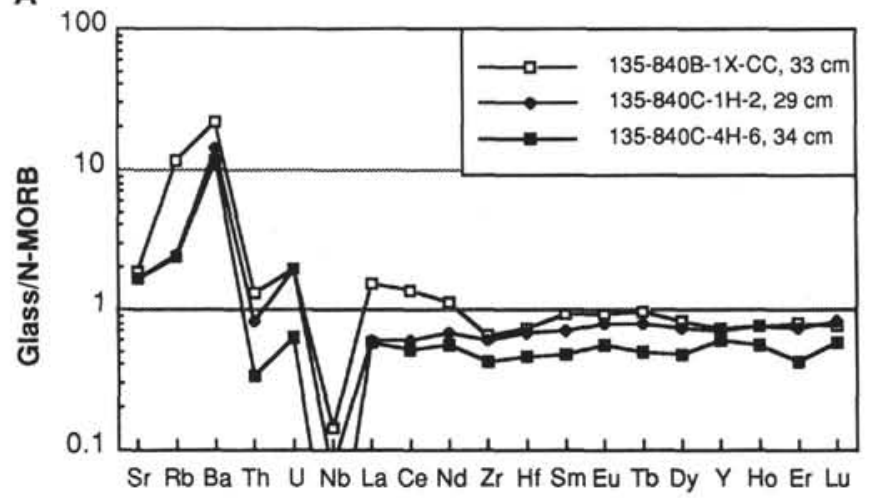

B

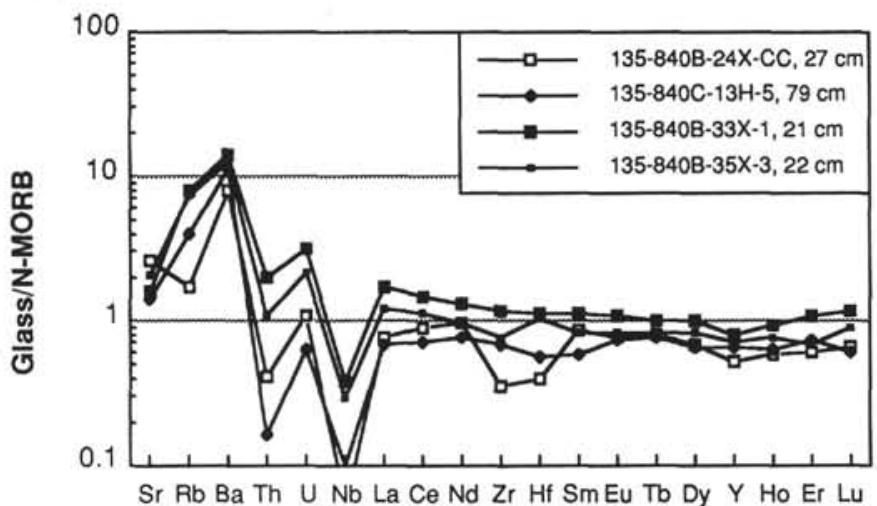

C

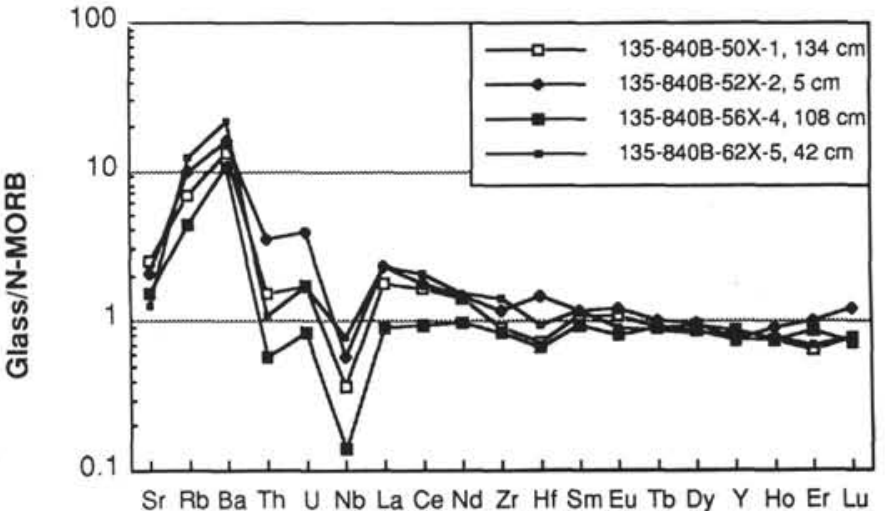

Figure 15. Spider diagrams of basaltic glass grains from Site 840 , showing the subduction zone enrichment in mobile elements and the depletion in $\mathrm{Nb}$. Note the consistent shape of the diagrams throughout the period of arc rifting and basin formation. A. Late Miocene. B. Uppermost late Miocene. C. Late Pliocene-middle Pleistocene.

Finally, it is likely that the Pleistocene airfall ash layers found within pelagic sediments at each site were derived from a number of subaerial volcanoes along the Tofua Arc. This is broadly in agreement with the variety of $\mathrm{Zr} / \mathrm{Nb}$ ratios found in basaltic fragments in these layers, and thus that various degrees of source depletion can be found during this time interval. Ewart and Hawkesworth (1987) demonstrated that this ratio, and thus the HFSE depletion of the island-arc volcanics, varies systematically along the arc in the present day. The most depleted lavas with the highest $\mathrm{Zr} / \mathrm{Nb}$ ratios are found in the northern Tofua Arc, whereas less depleted rocks occur in the south. Both types are thought to produce widespread ash layers in the 


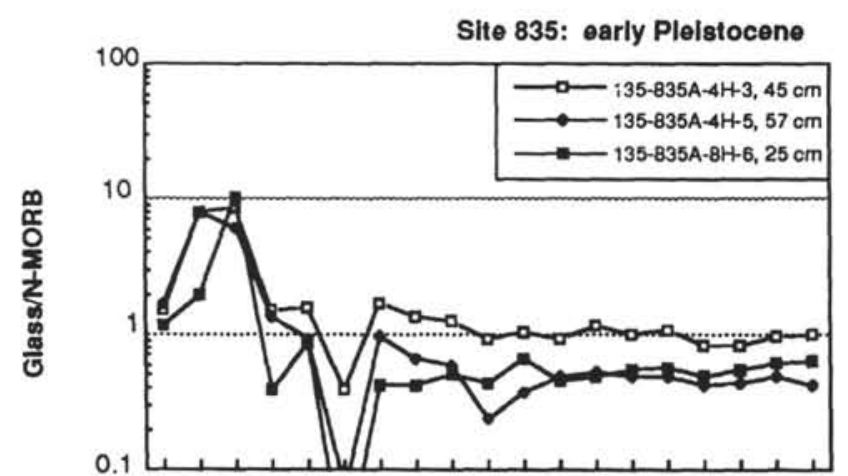

Sr RbBa Th U Nb La Cend ZrHi SmEu Tb Dy Y HoEr L

Site 837: early-middle Pleistocene

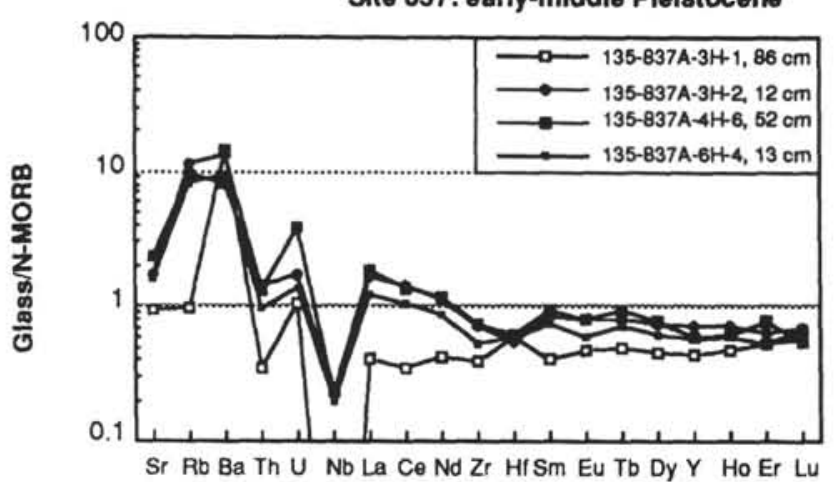

Site 839: early Pleistocene

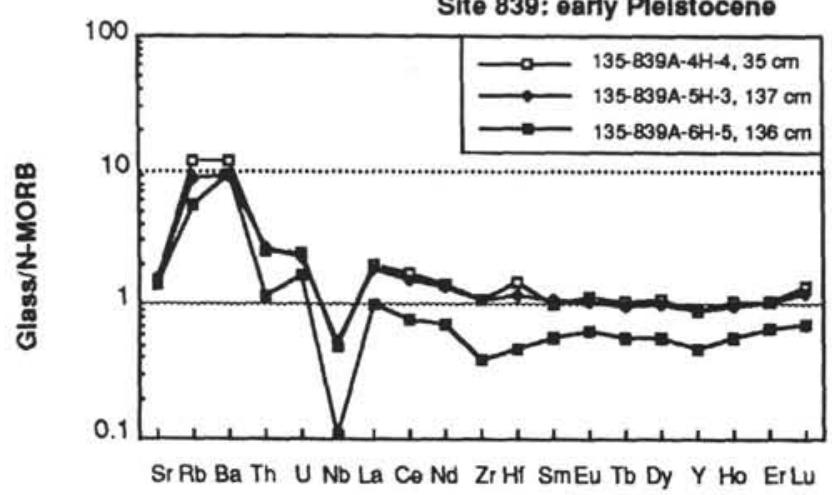

Site 835: late Pllocene

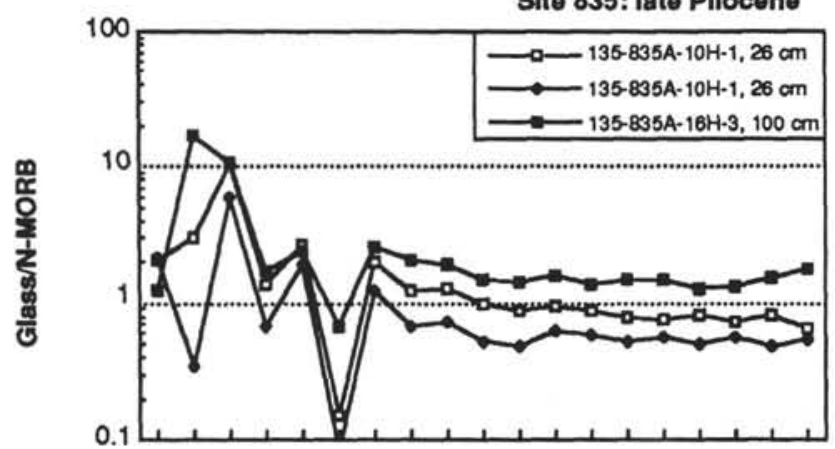

SrRb Ba Th U Nb LaCoNd Zr HISmEu To Dy Y Ho ErLu

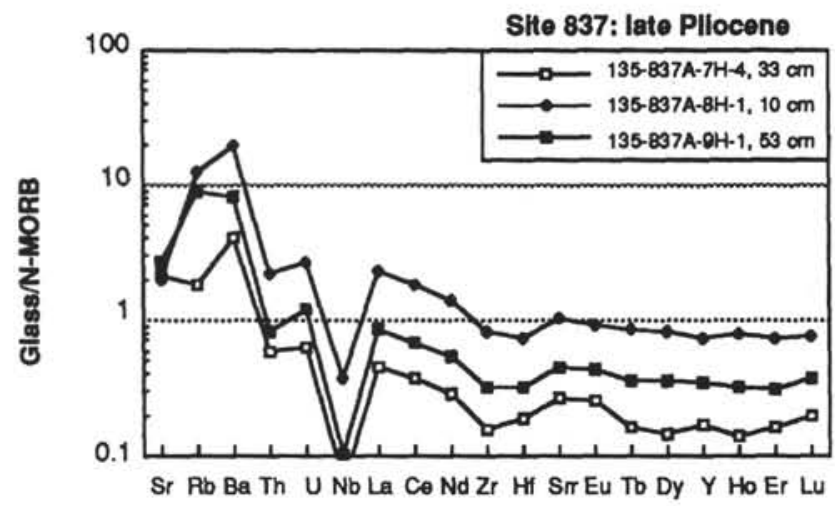

Shte 839: late Pllocene

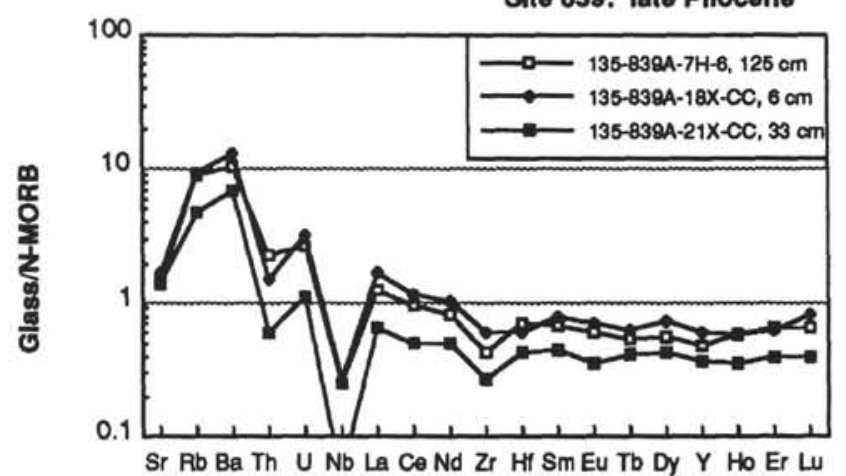

Figure 16. Spider diagrams of basaltic glass grains from Sites 835,837 and 839 showing the flat immobile element trend and subduction zone overprint also found at Site 840 .

backarc basin during the Pleistocene. It is this along-strike variability that can explain the spread of values of $\mathrm{Zr} / \mathrm{Nb}$ in the airfall ash layers.

\section{HISTORY OF ARC VOLCANISM}

Site 840 and the proximal volcaniclastic debris at Sites 834-839 provide a well-dated example of how the Tonga Arc system responds volcanically to the processes of rifting and marginal basin formation. Sedimentary and subsidence analyses of the Tonga Platform indicate rapid extension and subsidence during the initial stages of rifting (Austin et al., 1989; Clift, this volume). Shortly after the initial phase of arc rifting volcaniclastic sedimentation at Site 840 reached a minimum and is interpreted to indicate the cessation of volcanism on the east side of the new Lau Basin. The voluminous volcaniclastic sediments deposited at Site 840 during the initial rifting may reflect sedimentation from fault scarps in a tectonically active setting. Alternatively, the volcanic conglomerates may indicate a final, voluminous phase of volcanism of the Miocene Tonga Arc (Lau Ridge) before separation from the Tonga Platform. Voluminous volcanism is known from a similar rifting arc environment in the Sumisu rift of the Izu-Bonin Arc (Nishimura et al., 1991). The small size of the modern Tofua Arc and the minimum in volcaniclastic sedimentation between 5.0 and 3.0 Ma recorded at Site 840 suggest that island arc volcanism may have only established itself on the eastern side of the basin during the past $3.0 \mathrm{~m}$.y. If this is true then the limited amount of volcaniclastic sediment deposited at Site 840 during the 5.0-3.0 Ma period must have been derived by airfall from the still active Lau Ridge. It is thought unlikely that submarine seamount volcanoes in the new basin would have been capable of deriving significant amounts of debris out of the basin and on to the Tonga Platform. Thus, before the foun- 
A

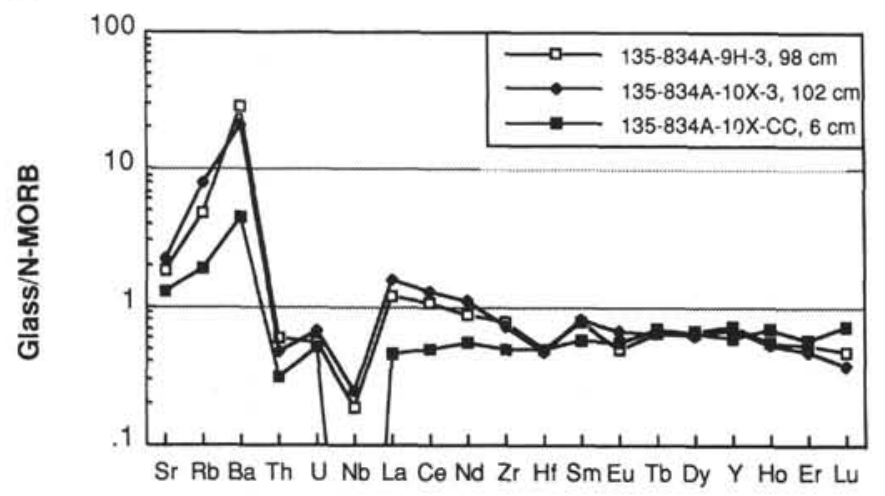

B

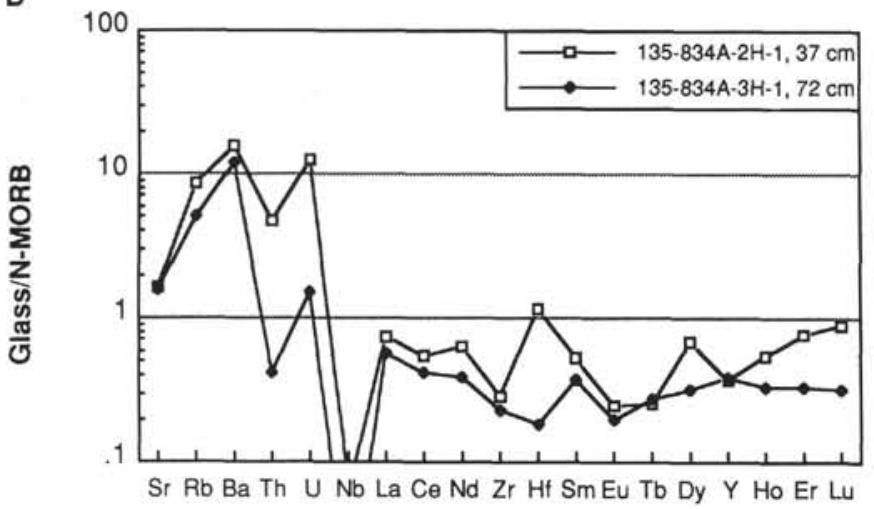

Figure 17. Spider diagrams of basaltic glass grains from Site 834 showing the change in glass composition since initial arc rifting. Source of Pleistocene glass debris does not correlate with the active Tofua Arc. A. Early Pliocene. B. Pleistocene.

dation of the new Tofua Arc, volcanic activity at large intrabasinal tholeiitic seamounts may, in effect, be considered to represent the arc volcanism during the early stages of basin rifting.

\section{A MODEL OF VOLCANICLASTIC SEDIMENTATION}

The chemical evidence cited above points to a majority of the volcaniclastic sediment in the Lau Basin being derived over short distances from seamount volcanos active while each sub-basin was rifting (Fig. 31). During the Pleistocene small volumes of sediment were derived by airfall directly from the Tofua Arc. The Tofua Arc cannot have been a supplier of sediment for much of the earlier history of the Lau Basin, as its small size and the lack of large volumes of volcaniclastic debris on the adjacent Tonga Platform during the 5.0-3.0 Ma period suggest that the arc is only 3.0 m.y. old. Even today when the Tofua Arc is as big as it has ever been, the amount of material shed into the basin is small. That much of the sediment is probably derived quite locally from volcanically active seamounts is apparent from the rugged, fault-bounded sub-basins that characterize the Lau Basin. Because the topography acts as a very efficient sediment barrier, long-distance sediment transport along or across the basin axis would have been impossible. The partially filled nature of each sub-basin means that water-suspended volcaniclastic material, either in the form of proximal turbidites or mass-flow deposits, once eroded from any one volcano (or from the Lau Ridge or Tofua Arc) will tend to pond around its source. Although submarine eruptions in the form of eruptive plumes (Fiske and Matsuda, 1964) may carry material further than a turbidity current in a region of rough topography, the distance over which coarse volcanic debris is likely to be carried is still relatively
A

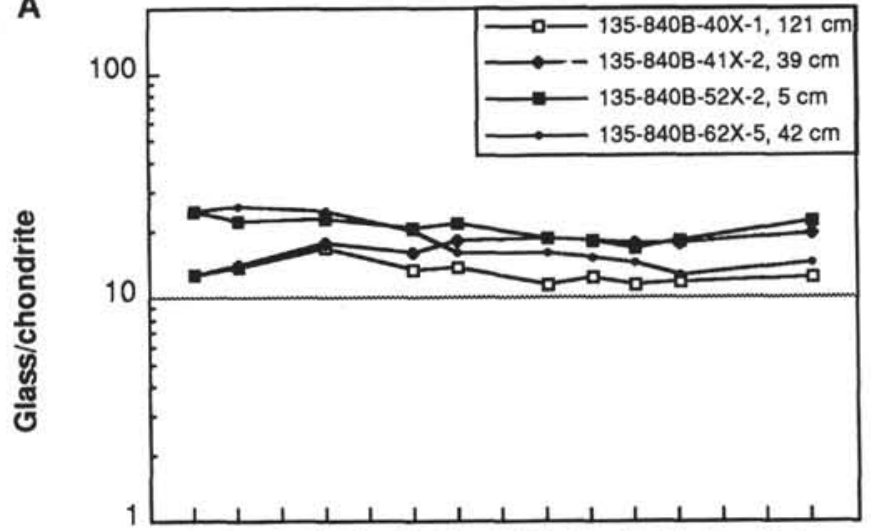

La Ce Pr Nd Pm Sm Eu Gd Tb Dy Ho Er Tm Yb Lu

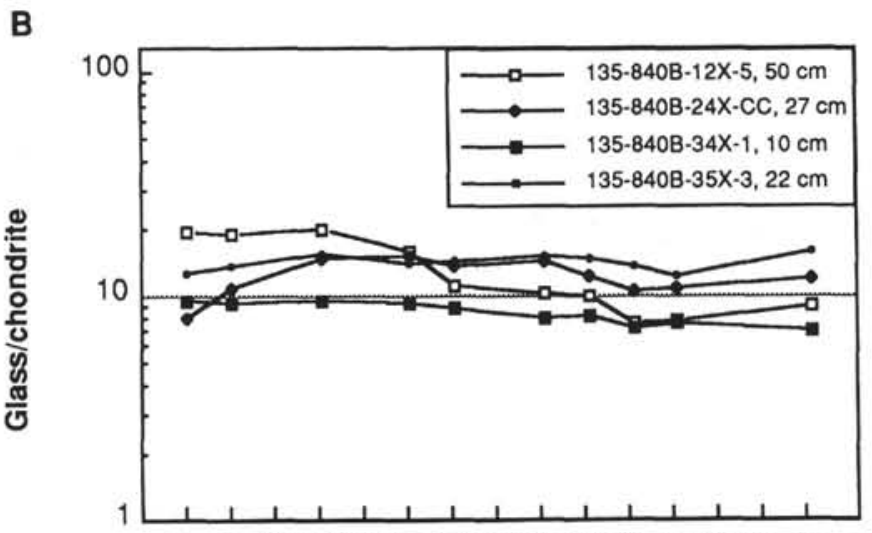

La Ce Pr Nd Pm Sm Eu Gd Tb Dy Ho Er Tm Yb Lu

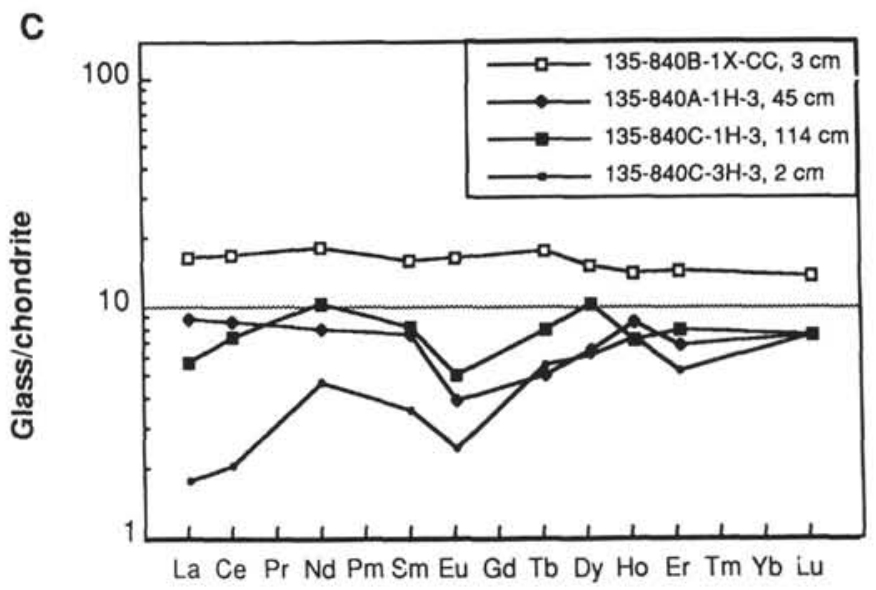

Figure 18. Chondrite normalized rare earth element diagrams for basaltic andesite grains from Site 840 (Tonga Platform). A. Late Miocene. B. From the uppermost late Miocene. C. Late Pliocene-middle Pleistocene. Normalizing values are from Sun and McDonough (1989).

modest (approximately $20 \mathrm{~km}$ maximum), a prediction reflected in the major, trace, and rare earth element chemistry presented. As far as the origin of the airfall ash layers deposited during the Pleistocene is concerned, it seems likely that contributions at any one sub-basin come from a number of volcanoes along the length of the arc (Fig. 32).

Of critical importance is the realization that the style of sedimentation in the basin is linked to the tectonic style and nature of the 


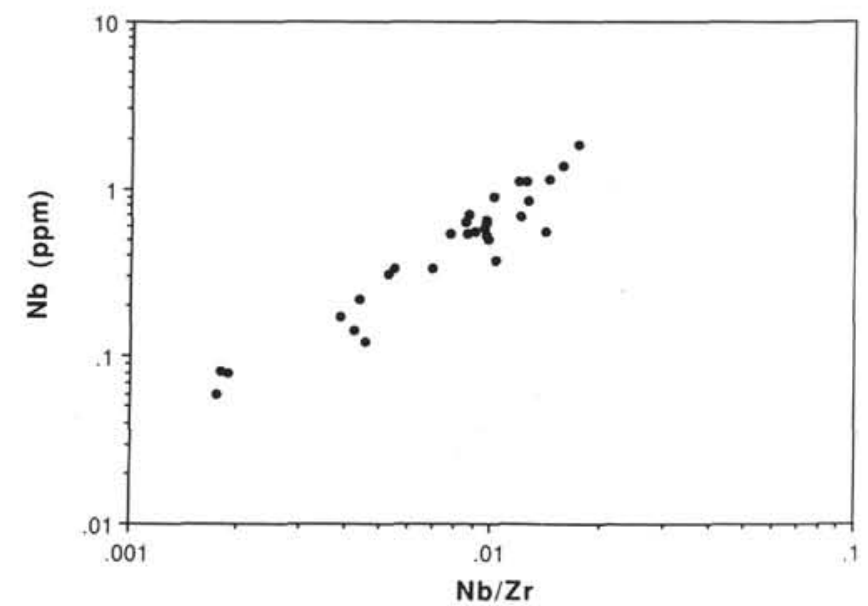

Figure 19. Diagram showing the relationship of $\mathrm{Nb} / \mathrm{Zr}$ to $\mathrm{Nb}$ at Site 840 .

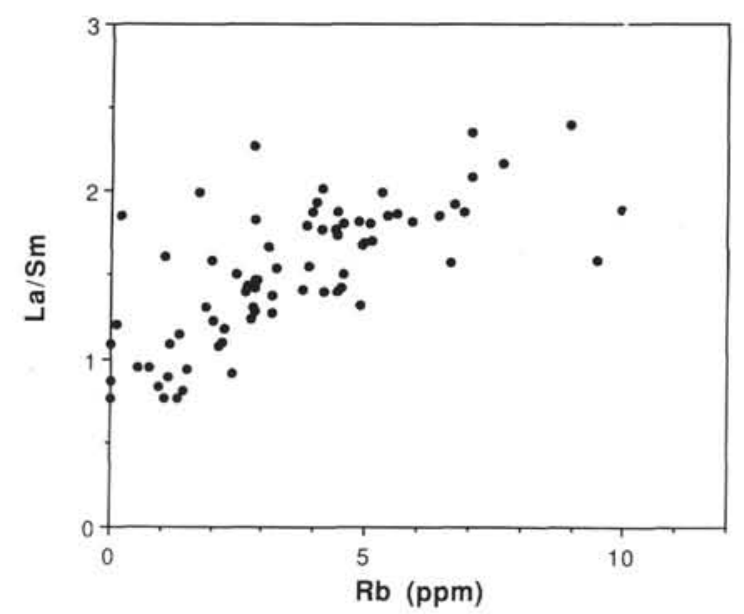

Figure 20. Diagram showing the strong positive correlation between $\mathrm{Rb}$ and LREE enrichment as measured by La/Sm. Data taken from Sites 834 to 840 .

volcanism. The changeover upsection at each site from proximal mass-flows and turbidites to pelagic oozes with ash intervals is linked to the migration of the zone of active extension within the Lau Basin to the east. The start of spreading on the Eastern Lau Spreading Center and the cessation of silica bimodal seamount volcanism also causes a changeover in the nature of proximal sedimentation at the start of sub-basin formation. Basins founded on extended arc crust are characterized by dacite-dominated turbidites and mass flows, compared to basaltic hyaloclastite breccias in basins formed along the Eastern Lau Spreading Center.

One important consequence of this model is that most of the sediment accumulating, both as primary ash layers and as reworked volcaniclastic turbidites and mass-flows, will tend to reflect the active volcanism at any one time over a relatively small area. The time delay between initial eruption and redeposition is seen to be geologically small. Apart from its importance to the interpretation of volcanic sediments in the Lau Basin, this model may also have significance for the interpretation of volcaniclastic sequences now found associated to ancient ophiolites (e.g., Dunnage Zone of Newfoundland, Josephine Ophiolite of the western United States, and the Sabah Ophiolite of Malaysia).

\section{ACKNOWLEDGMENTS}

We should like to thank Stuart Kearns and Pete Hill for their help and advice in the use of the Camebax Microbeam and John Craven and

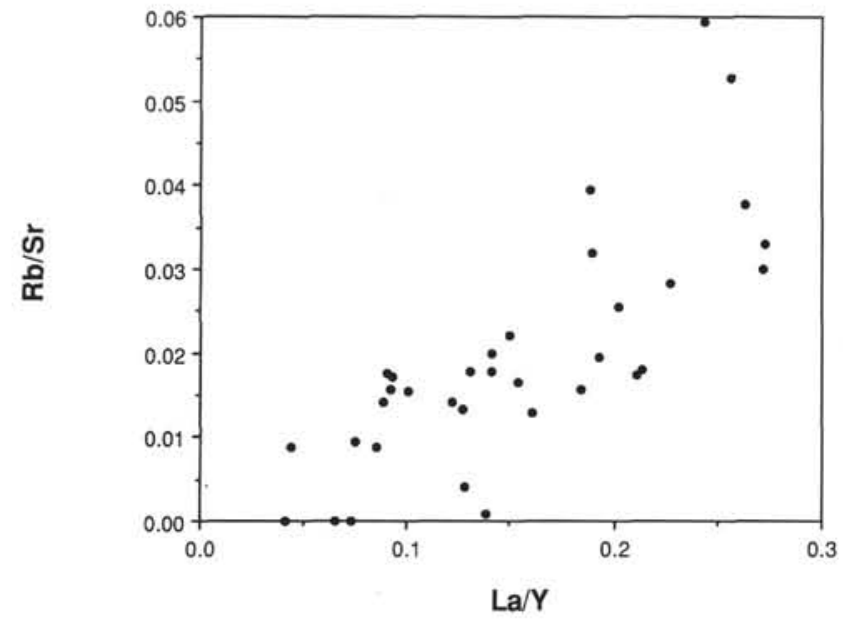

Figure 21. Diagram showing the variation of $\mathrm{La} / \mathrm{Y}$ with $\mathrm{Rb} / \mathrm{Sr}$ at Site 840 .

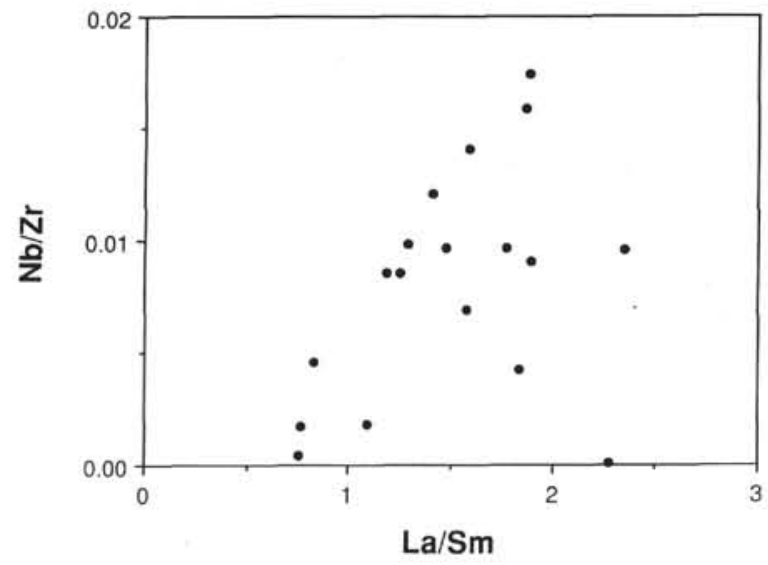

Figure 22. Diagram showing the variations of $\mathrm{Nb} / \mathrm{Zr}$ with $\mathrm{La} / \mathrm{Sm}$ at Site 840 . Only samples with $\mathrm{MgO}>4.0 \%$ are plotted.

Richard Hinton for their patient supervision of my time on the Cameca ion probe, both at Edinburgh University. We also thank Godfrey Fitton, Janet Hergt, Tony Ewart, Jim Hawkins, Lindsay Parson, and Julian Pearce for providing stimulating discussion on the nature of subduction-related magmatism and tectonics. P.C.'s work was completed as part of a British Petroleum/Royal Society of Edinburgh research fellowship. The authors thank Terry Plank and an anonymous reviewer for their helpful comments in improving this paper.

\section{REFERENCES}

Austin, J., Taylor, F.W., and Cagle, C.D., 1989. Seismic stratigraphy of the Central Tonga Ridge. Mar. Pet. Geol., 6:71-92.

Burnham, C.W., and Jahns, R.H., 1962. A method of determining the solubility of water in silicate melts. Am. J. Sci., 260:721-745.

Burns, R., and Andrews, J., 1973. Regional aspects of drilling in the southwest Pacific. In Burns, R.E., Andrews, J.E., et al., Init. Repts. DSDP, 21: Washington (U.S. Govt. Printing Office), 897-906.

Cawood, P.A., 1991. Nature and record of igneous activity in the Tonga arc, SW Pacific, deduced from the phase chemistry of derived detrital grains. In Morton, A.C., Todd, S.P., and Haughton, P.D.W. (Eds.), Developments in Sedimentary Provenance Studies. Geol. Soc. Spec. Publ. London, $57: 305-321$.

\footnotetext{
Abbreviations for names of organizations and publication titles in ODP reference lists follow the style given in Chemical Abstracts Service Source Index (published by
} American Chemical Society). 
Church, B.N., and Johnson, W.M., 1980. Calculation of the refractive index of silicate glasses from chemical composition. Geol. Soc. Am. Bull., 91:619 625 .

Cole, J.W., Gill, J.B., and Woodhall, D., 1985. Petrologic history of the Lau Ridge, Fiji. In Scholl, D.W., and Vallier, T.L. (Eds.), Geology and Offshore Resources of Pacific Island Arcs-Tonga Region. Circum-Pac. Counc. Energy Miner. Resour., Earth Sci. Ser., 2:379-414.

Ewart, A., 1982. The mineralogy and petrology of Tertiary-Recent orogenic volcanic rocks: with special reference to the andesitic-basaltic compositional range. In Thorpe, R.S. (Ed.), Andesites: Orogenic Andesites and Related Rocks: Chichester (Wiley), 26-87.

Ewart, A., Bryan, W.B., and Gill, J., 1973. Mineralogy and geochemistry of the younger volcanic islands of Tonga, southwest Pacific. J. Petrol., $14: 429-465$.

Ewart, A., and Hawkesworth, C.J., 1987. The Pleistocene-Recent TongaKermadec arc lavas: interpretation of new isotope and rare earth data in terms of a depleted mantle source model. J. Petrol., 28:495-530.

Fiske, R.S., and Matsuda, T., 1964. Submarine equivalents of ash flows in the Tokiwa Formation, Japan. Am. J. Sci., 262:76-106.

Gill, J.B., Stork, A.L., and Whelan, P.M., 1984. Volcanism accompanying backarc basin development in the Southwest Pacific. Tectonophysics, 102:207-224.

Hawkins, J.W., Bloomer, S.H., Evans, C.A., and Melchior, J.T., 1984. Evolution of intra-oceanic arc-trench systems. Tectonophysics, 102:175-205.

Hawkins, J.W., and Melchior, J.T., 1985. Petrology of Mariana Trough and Lau Basin basalts. J. Geophys. Res., 90:11431-11468.

Hinton, R.W., and Upton, B.G.J., 1991. The chemistry of Zircon: variations within and between large crystals from syenite and alkali basalt xenoliths. Geochim. Cosmochim. Acta, 55:3287-3302.

Irvine, T.N., and Baragar, W.R.A., 1971. A guide to the chemical classification of the common volcanic rocks. Can. J. Earth Sci., 8:523-548.

Karig, D.E., 1970. Ridges and basins of the Tonga-Kermadec island arc system. J. Geophys. Res., 75:239-254.

Latin, D.M., Dixon, J.E., and Fitton, J.G., 1990. Rift-related magmatism in the North Sea Basin. In Blundell, D.J., and Gibbs, A. (Eds.), Tectonic Evolution of the North Sea Rifts: Oxford (Oxford Univ. Press), 102-144.

Le Bas, M.J., 1962. The role of aluminum in igneous clinopyroxenes with relation to their parentage. Am. J. Sci., 260:267-288.

McKenzie, D., and Bickle, M.J., 1988. The volume and composition of melt generated by extension of the lithosphere. J. Petrol., 29:625-679.

Moberly, R., 1971. Youthful oceanic lithosphere of marginal isles, western Pacific. Proc. 12th Pac. Sci. Congr., Canberra.

Neilson, C.H., and Sigurdsson, H., 1981. Quantitative methods for electron microprobe analysis of sodium in natural and synthetic glasses. Am. Mineral., 66:547-552.

Nishimura, A., Marsaglia, K.M., Rodolfo, K.S., Colella, K.S., Hiscott, R.N., Tazaki, K., Gill, J.B., Janecek, T., Firth, J., Isilinger-Kelso, M., Herman, Y., Taylor, R.N., Taylor, B., and Fujioka, K., 1991. Pliocene-Quaternary submarine pumice deposits in the Sumisu rift area, Isu-Bonin Arc. In
Fisher, R.V., and Smith, G.A. (Eds.), Sedimentation in Volcanic Settings. Spec. Publ.-Soc. Econ. Paleontol. Mineral., 45:201-208.

Parson, L., Hawkins, J., Allan, J., et al., 1992. Proc. ODP, Init. Repts., 135: College Station, TX (Ocean Drilling Program).

Parson, L.M., Hawkins, J.W., and Hunter, P.M., 1992. Morphotectonics of the Lau Basin seafloor-implications for the opening history of backarc basins. In Parson, L., Hawkins, J., Allan, J., et al., Proc. ODP, Init. Repts. 135: College Station, TX (Ocean Drilling Program), 81-82.

Parson, L.M., Pearce, J.A., Murton, B.J., Hodkinson, R.A., Bloomer, S., Ernewein, M., Huggett, Q.J., Miller, S., Johnson, L., Rodda, P., and Helu, S., 1990. Role of ridge jumps and ridge propagation in the tectonic evolution of the Lau back-arc basin, southwest Pacific. Geology, 18:470-473.

Pearce, J.A., 1982. Trace element characteristics of lavas from destructive plate boundaries. In Thorpe, R.S. (Ed.), Andesites: New York (Wiley), 57-75.

1983. The role of sub-continental lithosphere in magma genesis at destructive plate margins. In Hawkesworth, C.J., and Norry, M.J. (Eds.), Continental Basalts and Mantle Xenoliths: Nantwich, UK (Shiva), 230-249.

Peccerillo, A., and Taylor, S.R., 1976. Geochemistry of Eocene calc-alkaline volcanic rocks from the Kastamonu area, northern Turkey. Contrib. Mineral. Petrol., 58:63-81.

Scholl, D.W., Vallier, T.L., and Maung, T.U., 1985. Introduction. In Scholl, D.W., and Vallier, T.L. (Eds.), Geology and Offshore Resources of the Pacific Island Arcs-Tonga Region. Circum-Pac. Counc. Energy Miner. Resour., Earth Sci. Ser., 2:3-15.

Simonian, K.O., 1975. The geology of the Arakapas Fault Belt Area, Troodos Massif, Cyprus [Ph.D. thesis]. Open Univ.

Spiegelman, M., and McKenzie, D.P., 1987. Simple 2-D models for melt extraction at mid-ocean ridges and island arcs. Earth Planet. Sci. Lett., $83: 137-152$

Sun, S.-S., and McDonough, W.F., 1989. Chemical and isotopic systematics of oceanic basalts: implications for mantle composition and processes. In Saunders, A.D., and Norry, M.J. (Eds.), Magmatism in the Ocean Basins. Geol. Soc. Spec. Publ. London, 42:313-345.

Tappin, D.R., 1992. The Tonga Forearc Basin. In Ballance, P.F., Herzer, R.H., and Stevenson, A.J. (Eds.), Geology and Resources of Island Arcs, TongaLau-Fiji Region: Berlin (Springer-Verlag).

Weissel, J.K., and Watts, A.B., 1975. Tectonic complexities in the south Fiji marginal basin. Earth Planet. Sci. Lett., 28:121-126.

Woodhall, D., 1985. Geology of the Lau Ridge. In Scholl, D., and Vallier, T.L. (Eds.), Geology and Offshore Resources of Pacific Island Arcs-Tonga Region. Circum-Pac. Counc. Energy Miner. Resour., Earth Sci. Ser. $2: 351-378$.

Date of initial receipt: 29 June 1992

Date of acceptance: 7 June 1993

Ms 135SR-102 


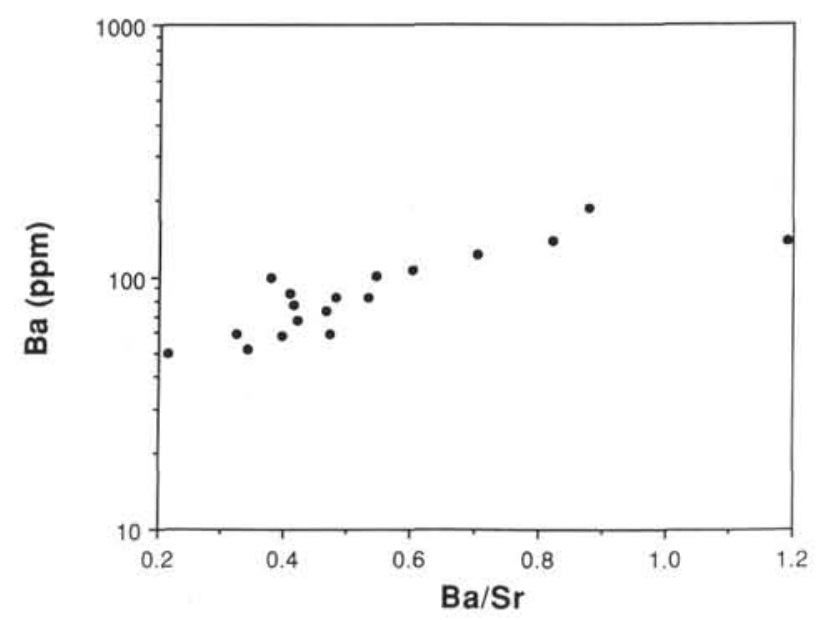

Figure 23. Diagram showing the variation of $\mathrm{Ba} / \mathrm{Sr}$ with $\mathrm{Ba}$ at Site 840 . Only samples with $\mathrm{MgO}>4.0 \%$ are plotted.
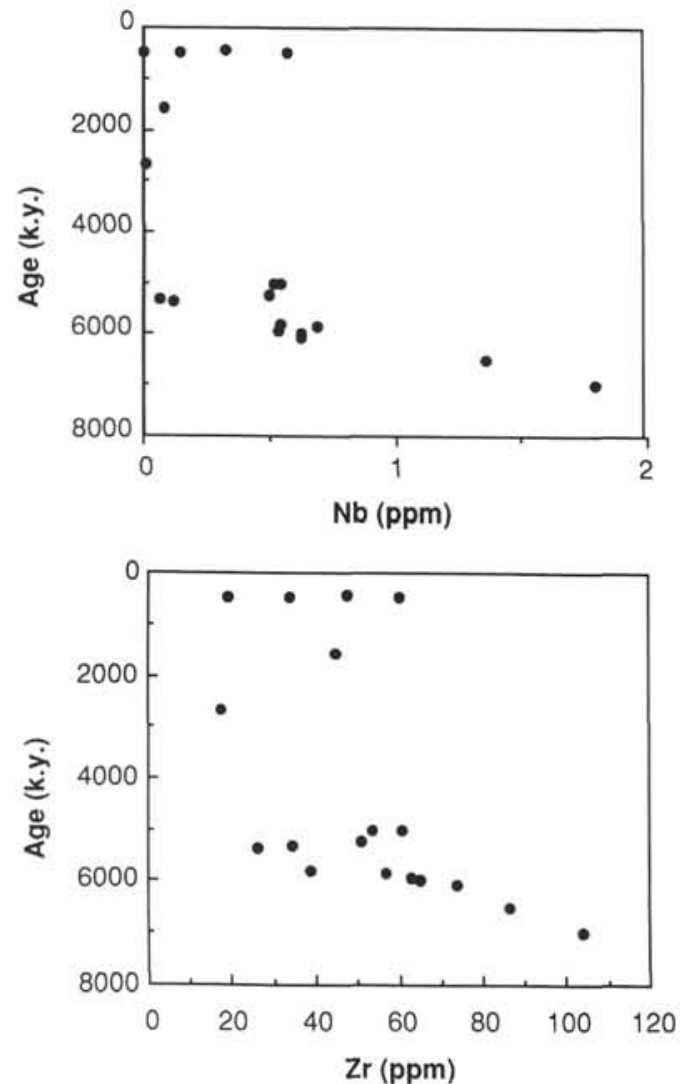

Figure 24. Diagram showing the variation in total $\mathrm{Nb}(\mathbf{A})$ and $\mathrm{Zr}(\mathbf{B})$ concentrations with time at Site 840 , Tonga Platform.

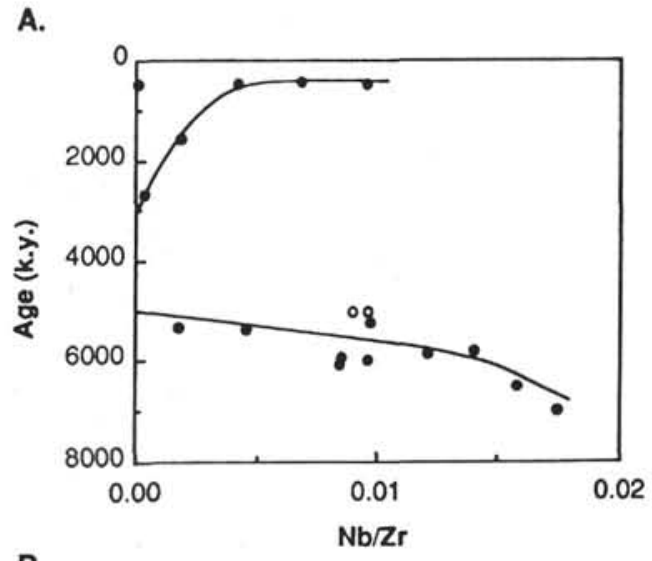

B.

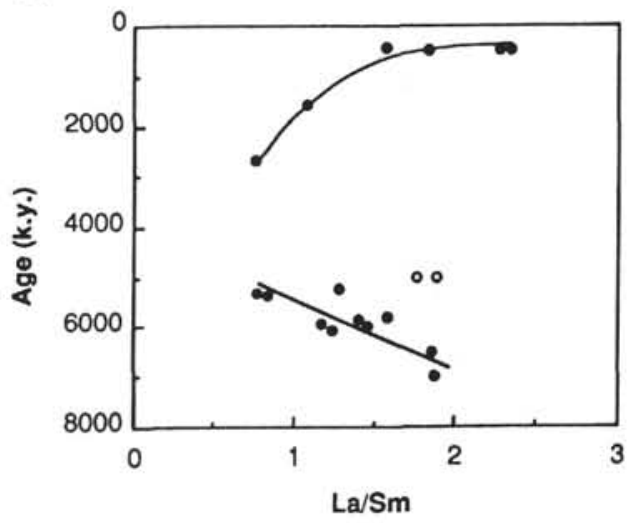

C.

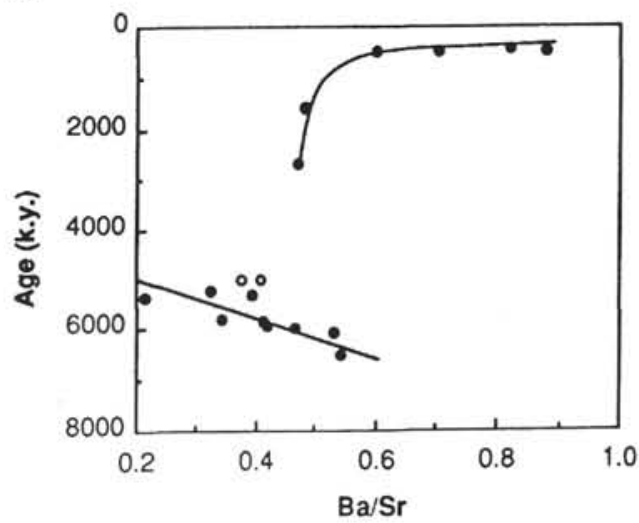

Figure 25. Diagram showing the increase in $\mathrm{Nb} / \mathrm{Zr}(\mathbf{A}), \mathrm{La} / \mathrm{Sm}(\mathbf{B})$, and $\mathrm{Ba} / \mathrm{Sr}$ (C) with time before Lau Basin rifting at Site 840 . Analyses with $\mathrm{MgO}>4 \%$ only. Grains deposited at the time of rifting are marked with open circles; all other analyses are black circles. 


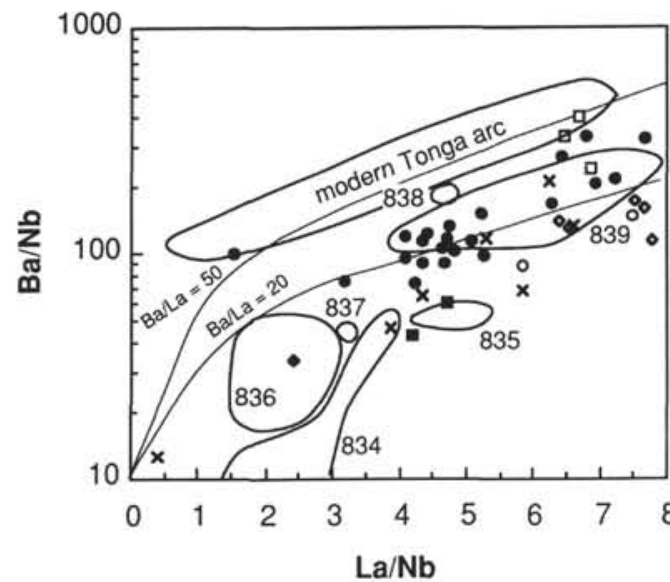

Figure 26. Diagram showing the changes in $\mathrm{Ba} / \mathrm{Nb}$ vs. $\mathrm{La} / \mathrm{Nb}$ for all basaltic glass grains from Sites 834 to $840(\mathrm{MgO}>4.0 \%)$. Fields show the distribution of analyses from basement basalts at Sites 834-839, together with analyses from the modern Tofua Arc (data from Ewart et al., this volume).

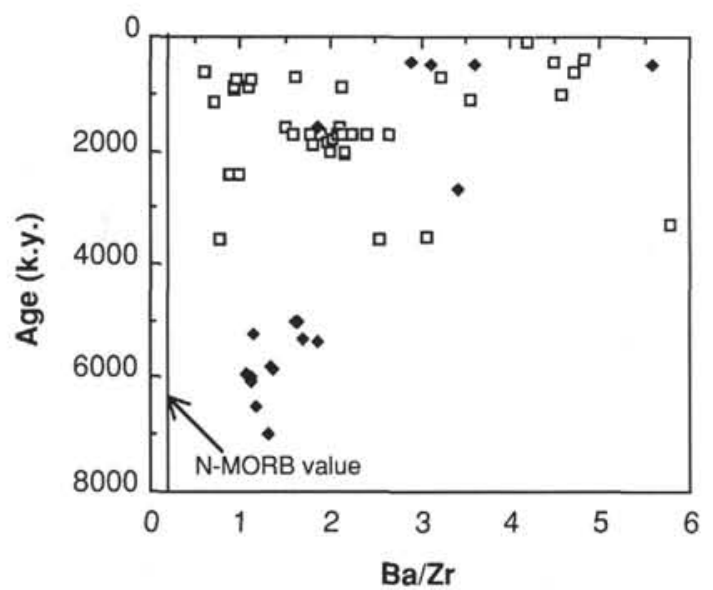

Figure 27. Variation in $\mathrm{Ba} / \mathrm{Zr}$ with time at Site 840 (solid diamond) and Site $834-839$ (open square). Note scatter of relatively low values before $5.0 \mathrm{Ma}$ and subsequent increase, suggestive of slab influence.

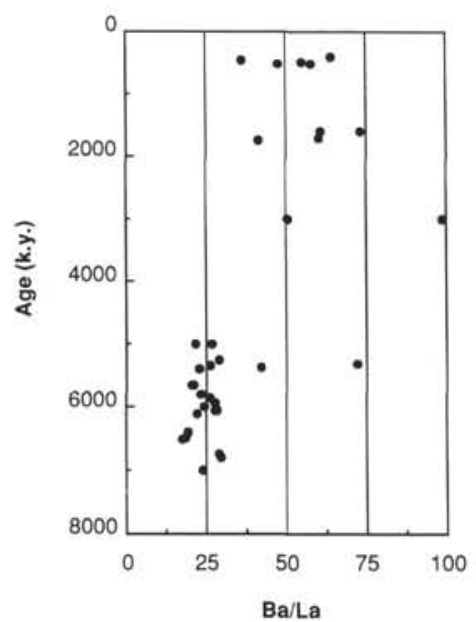

Figure 28. Diagram showing the variability of $\mathrm{Ba} / \mathrm{La}$ in silica-poor glasses at Site 840 with time. Note the low constant values of the prerift period compared with the higher values since $5.0 \mathrm{Ma}$.

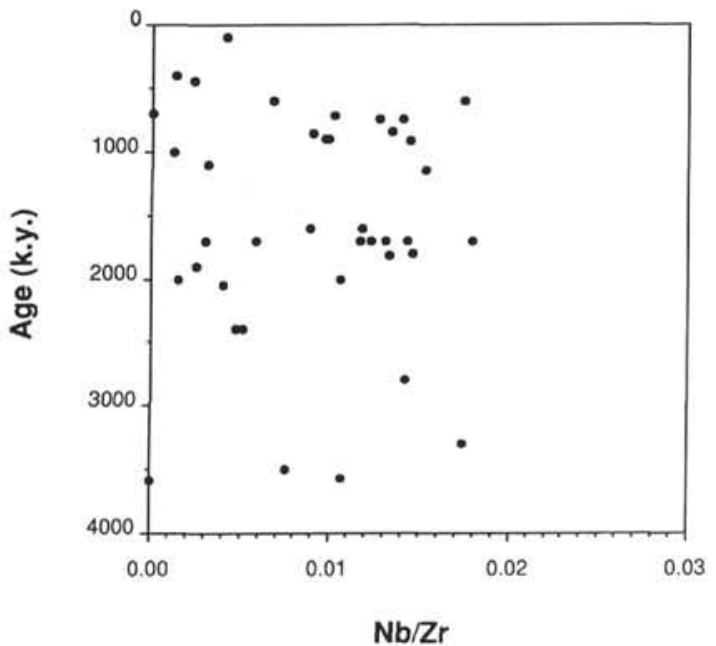

Figure 29. Diagram showing the spread of $\mathrm{Nb} / \mathrm{Zr}$ values for basaltic andesite glass grains from Sites 834 to 839 .

A

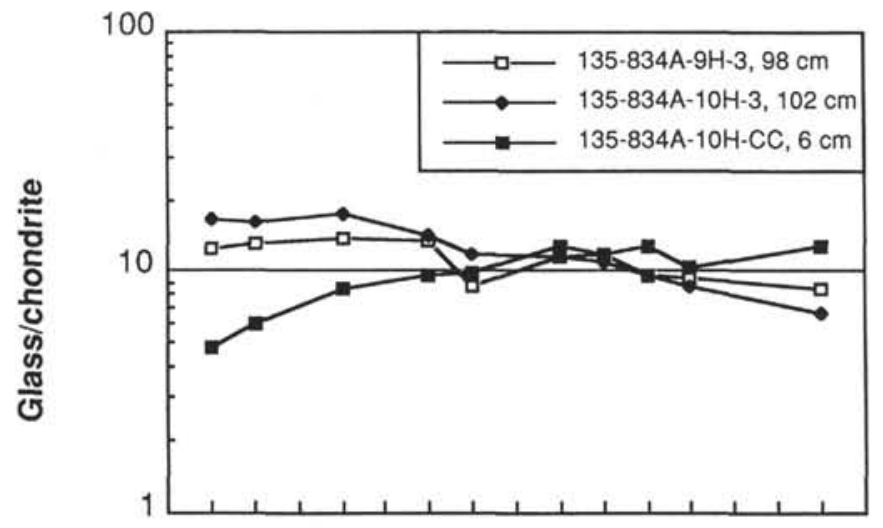

La Ce Pr Nd PmSm Eu Gd Tb Dy Ho Er Tm Yb Lu

B

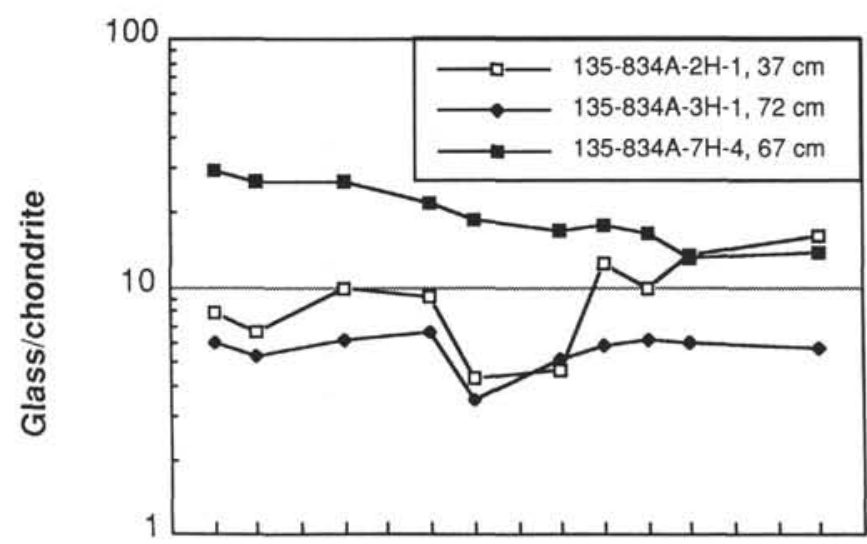

La Ce Pr Nd PmSmEu Gd Tb Dy Ho Er Tm Yb Lu

Figure 30. REE diagram of basaltic glass grains from Site 834 showing the change in glass composition since initial are rifting. A. Early Pliocene. B. Pleistocene. 
Late Miocene, 7.0 Ma-Steady state subduction

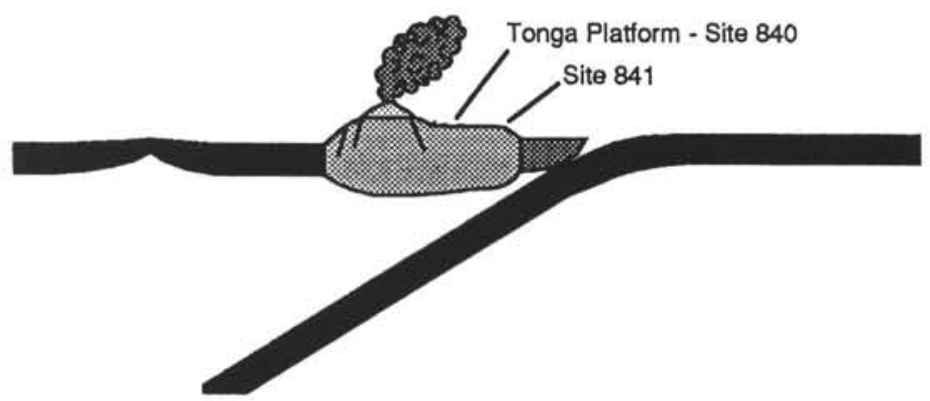

Late Miocene, 5.6 Ma - Extension of the arc

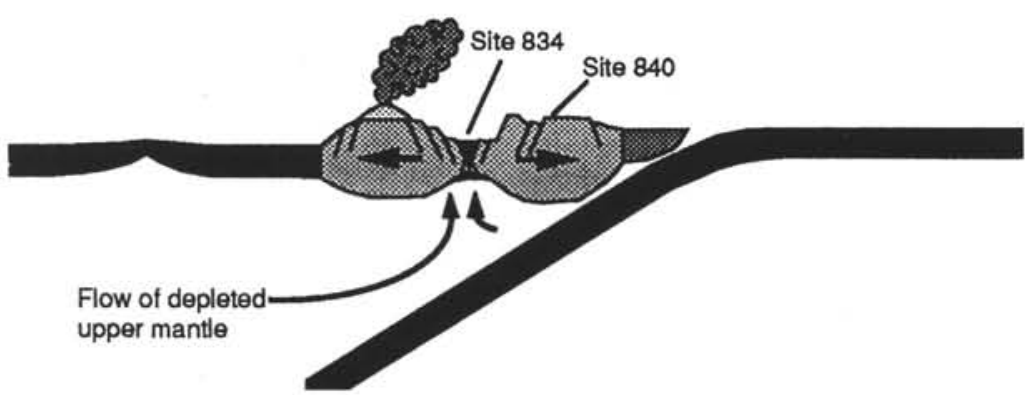

Middle Pliocene, 2.5 Ma - Continued extension and seamount volcanism

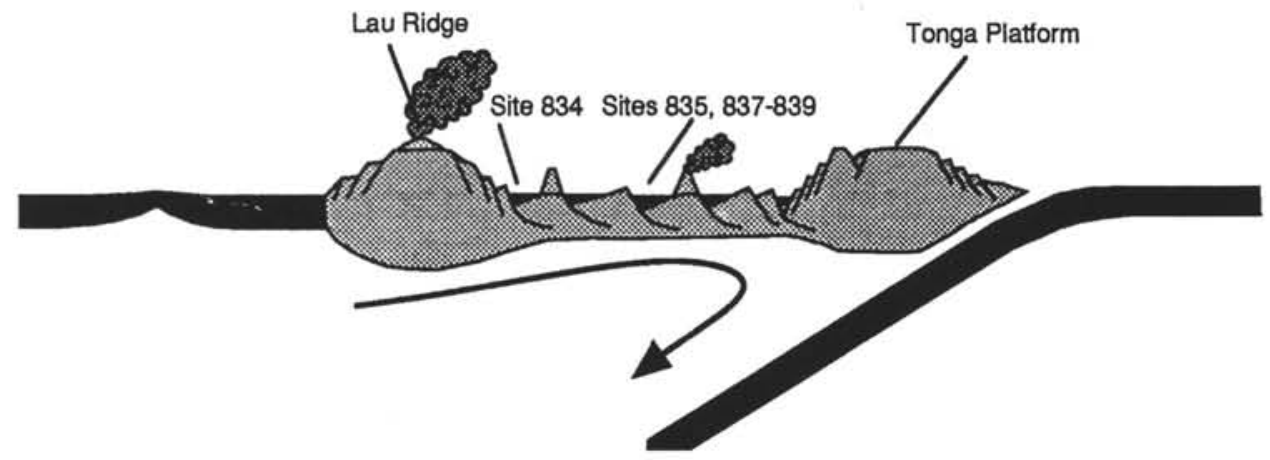

Late Pleistocene, 0.5 Ma - Spreading starts on Eastern Lau Spreading Center

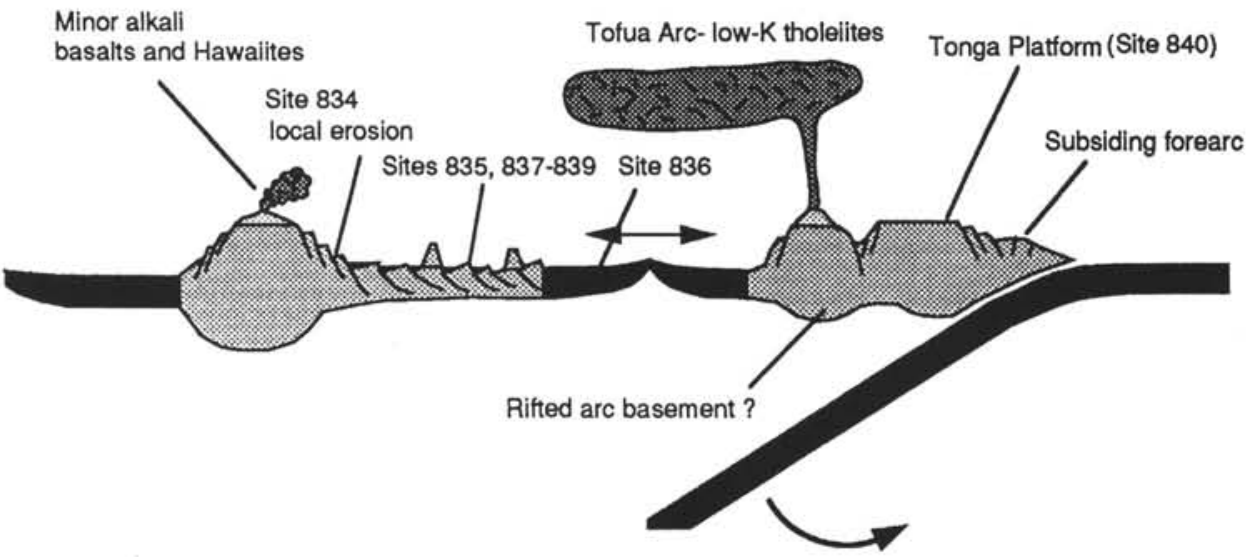

Figure 31. Diagrams showing changeover of sediment derivation from the Lau Ridge to intrabasinal seamounts during the opening of the basin and the inferred start of volcanism of the Tofua Arc in late Pliocene times. 


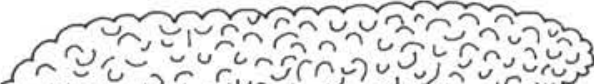

\{

$\mathrm{E}$

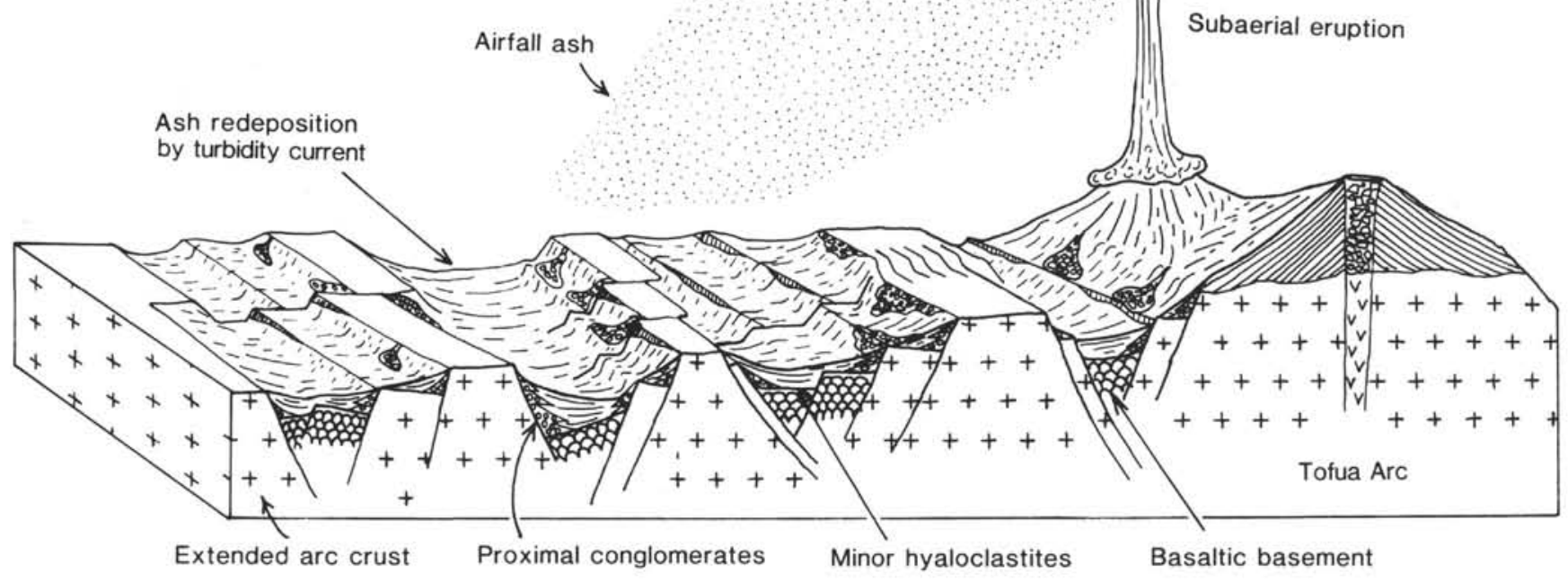

Figure 32. Favored model of volcaniclastic sedimentation during the Pleistocene involving deposition by airfall into the Lau Basin. Localized rapid sedimentation by submarine volcanic eruptions and their proximal reworking already finished by this time. 